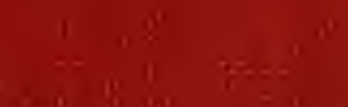

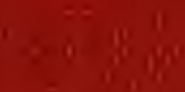
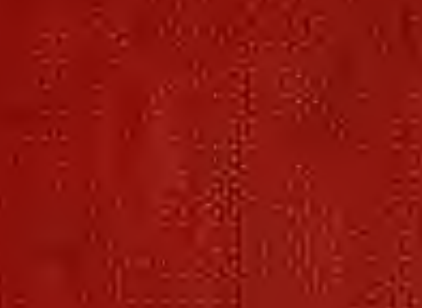

$10: 0000$

$6 y=-5=$ 


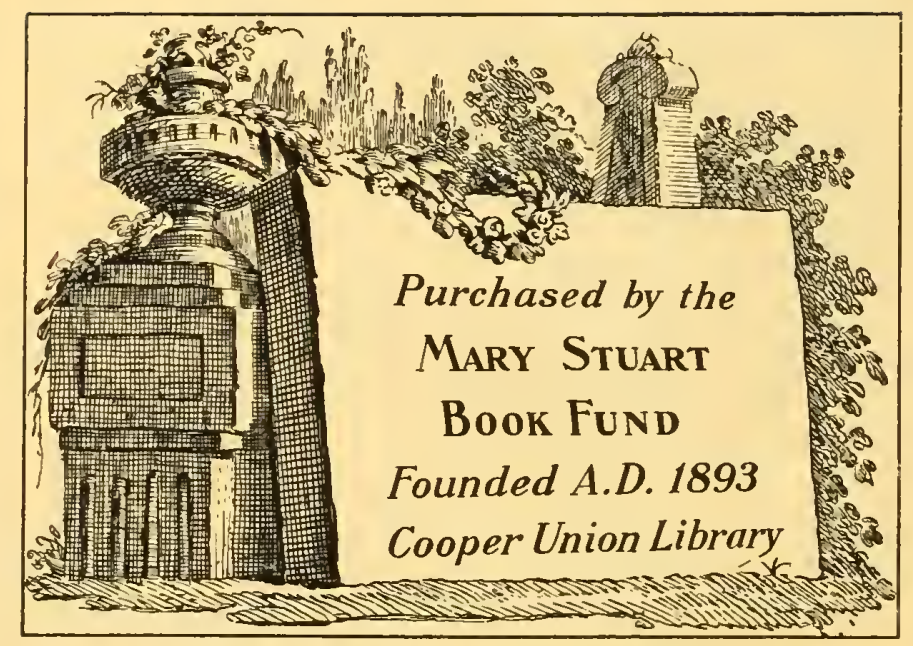


A mio Padre 


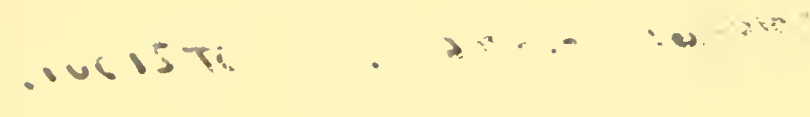




\section{GIORGIO SANGIORGI}

\section{CONTRIBUTI ALLO STUDIO DELL'ARTE TESSILE}

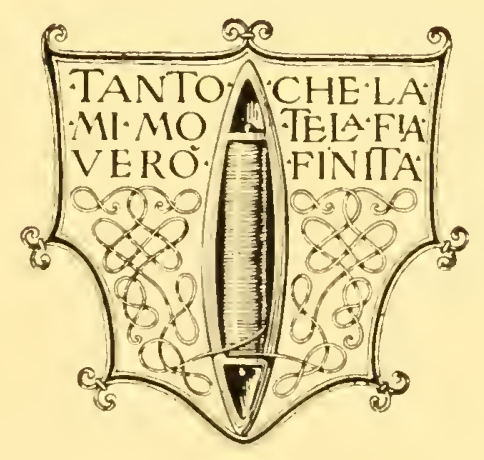

CASA EDITRICE D'ARTE BESTETTI \& TUMMINELLI MILA NO - ROMA 


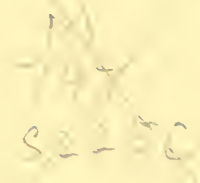




\section{A V VERTIMENTO}

L'intitolazione di questo libro dice e non promette piì di quanto intesero portare in contributo minimo allo studio dell arte tessile otto miei articoli pubblicati in questultimo lustro.

Dunque, non storia generale, nemmeno capitoli interi. bensi pagine sparse di un libro ancora da fare. Nella prima, che riferisco a mo" di prefazione da un mio diario este. tico, tento l'esegesi di una materia che prediligo ed elevo alle ragioni dell'arte; nella seconda adombro il metodo tecnico-analitico che terrò trattandola nelle seguenti, e che credo di avere utilmente inaugurato dal presupposto: che ogni veriti debba affermarsi col consenso dell'intimo all'estrinseco apparente. 


\section{DEL COLORE E DELLO STILE}

NELLE STOFFE ANTICHE 



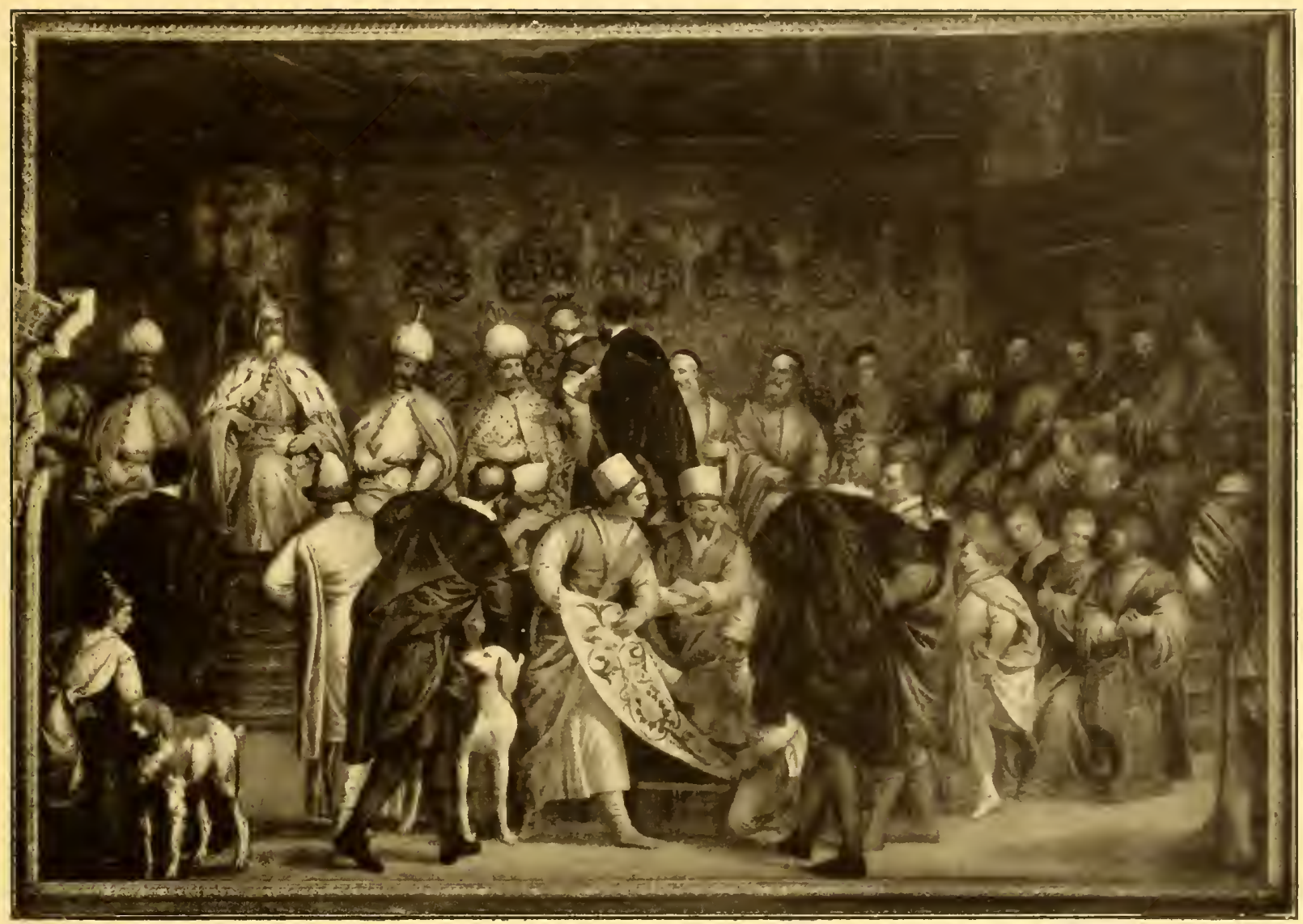

OFFERTA DI DONI TESSILI AL DOGE DI VENEZIA

\section{DEL COLORE E DELLO STILE NELLE STOFFE ANTICHE $\left(^{*}\right)$}

La mia sacrestia profana - come all'amico poeta piacque defunire il gnardaroha ove tengo quasi sempre chiuse le stoffe antiche che da anni vado raceogliendo - $\grave{e}$ in festa. I suoi cofani nuziali traboccano un tessile tesoro esotico e remoto di fluidi broccati, di sensuosi velluti, di vibratili bissi. di sete carezzevoli, di

Drappi contesti di porpore e d'oro,

Quasi manti di re fastosamente

Gittati alla rinfusa...

Ma prima di richindere le arehe mi piace indugiare sulle cose taciute in contemplazione, liberando alla luce tanta gloria di colore.

Fra le vibrazioni dell'etere cosmico, infunite in numero e velocità, locchio umano percepisee solo quelle che nel variare le apparenze della forma meglio concorrono alla

(*) Dalla Rassegna Italiana, anno 1919, fasc. XIX. 
illusione della vita. Infatti. se la luce fosse indissoluhile nei snoi elementi. negando alle cose la bella veste variopinta. lesistenza nostra sarcbbe come immersa in un al. bore monotono e triste forse quanto la continua notte del cieco. La gioia di vivere. come nel canto. si espande tutta nel tripudio delle tinte. dalle quali si rifugge sol quando ne vien meno. e al dolore cerchiamo rispondenza nella loro negazione.

Già il Goethe in Teoria dei Colori avvertì com'essi. i colori. agiscano sull'animo nostro suseitandovi sensazioni che ora ci riposano ora ci agitano fra la tristezza e la letizia: onde. nonchè convenuto, il lingnaggio simbolico delle linte. appare spontanea espres. sione del potere sensibile dell"iride sullo spirito. Comunque quanto potente stimolo sia il colore lo conferma praticamente quel senso di desolazione che ci prende come un in. cubo negli squallidi ambienti delle scuole, nelle caserme, negli ospedali. ove le scialbe inadorne pareti ammentano la nostalgia di quelle domestiche. negando allochio errabondo un punto di appoggio per riposare ricreandosi. Ma forse un giorno quando l'arte fosse alimento sociale non meno necessario del pane e dellacqua. detti asili si vorranno meno ingrati di adesso. come già in Inghilterra che si ricorre alla distrazione delle tinte per lenire lo spleen ai convalescenti.

Ed ora. rifacendoci alle remote origini del senso estetico. apprendiamo da Darwin che in alcuni animali il primo fascino è appunto per il colore. onde attraverso la selezione sessuale si produssero eapolavori di bellezza amaliatrice. e sono certi uccelli e certe farfalle esotici; le quali creature. se non nate a piacerei. incontrando la nostra anmirazione. dimustrano aver comune con noi la base sensoria del gusto. da eni sapemmo elevarei al privilegio dell arte disinteressata.

Qual dunque antichissima fonte edonistica. il eolore accende luomo primitivo di ammirazione infantile alla vista. non solo dei volatili e de’ fiori. bensi d'ogni cosa colo. rata; e per la gioia degli ocehi egli spinse la ricerea, nei progressivi stati di civiltà. funo alle viscere della terra per estrame le gemme che dall'eterna notte natale si destano a nuova vita nel veder la luce. Cosi sempre, dal Nord. ovegli interrompe con vibranti note del culto floreale la caligine perenne, al Sud, ove enstodisce allombra della dimora, nei tappeti. nei parati. nelle ceramiche e in ogn oggetto d'uso consueto, liride che fuori si discolve al sole, l’uomo dimostrò sempre di amarne la gioconda varietà nelle sue infinite espressioni naturali ed artistiche. E forse prima chegli si dilettasse nella contemplazione plastica delle cose, inquinata quasi sempre di spurii riflessi logici e considerazioni pratiche. fu più sensibile alla pura percezion cromatica: ma dal piacere primitivo che può destare una sol nota di colore - unità di senso - al multiplo sintetico delle più complesse sinfonie, quanto il cammino!

Partendo dalla Grecia arcaica, in difetto di documenti pittorici. vi supponiamo con l'ansilio della filologia. che suggerisce scarso ed improprio il lessico della gamma, debole pure il senso ottico del colore; ma già verso il secolo di Prassitele, anche dalla scultura stessa, comincia a trasparire nella sua indeterminazione impressionistica (mi si 
fassi il neologismo) il raffinarsi della sensibilita per esoo: la quale giunse poi di grarlo in grado fino ad esprimersi in forma estetica prevalente in sull aprire di quel medioevo. che

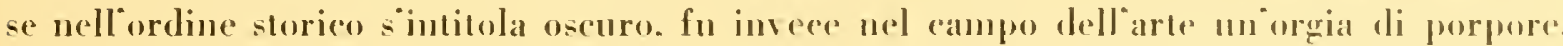

Contriburono alla metamorfosi le correnti artistiche e culturali a-iatiches già attive al nascente Impero: le fuali comineiarono appunto allora col minarme le fondamenta, funchè louda del colore, nata al sol levante. si diffuse per tutto looceidente, rifulgendo sulle ruine: e fu nettare alla nuova sete. che solo allalloa della Rinascenza comincio ad estinguersi: cosicchè all"epoca di Dante lanore barbarico frer i colori puri. è già morente in nostalgieo smarrimento di ombre e di luci fra il si a il no del hianeo e del nero. La stessa tavolozza letteraria del Poeta. al contrario dellomerioa. lo conferma: in essa ogni più delicata sfumatura dello spettro i tolta alle visioni naturalistiche per adombrare i moti dellanimo più tenui con vigile percezione es squisita sensibilita morale. Ritornava ormai il chiaro-seuro prassitelico. preludente. in ragionc inversa. al rigenerarsi della forma plastica. Così, ogni civiltà. ognepoca. dimostra aver avuta la propria lingua cromatica con la sua poesia.

Amato dal medioevo in gemmea purezza e nei snoi più vivari rontrasti. vi ritroviamo il eolore condensato nelle tessere de mosaici. circoscritto nel piombo delle vetrate. incastonato nelloro degli smalti. cotto sulle matioliche. intesinto nei tappeti e nelle stoffe: ovmuque profuso e non mai confuso.

E. fra le tante altre manifestazioni del hosso. furono sopratmuto le stoffe che più colorirono il medioevo: e specialmente in questo periodo. a similimdine della misteriosa volontà della natura che ricopre i volatili di smaglianti abiti nuziali. la vanità umana. animala anchiessa da un oscuro istinto sednttivo. chiede ausilio agli splendenti orpelli, onde meglio imperare. oltre che nel dominio dellamore. in quello politiro. Pereio non ci sorprenda se fun dai limiti dell'antichità. le fluide sete e gli ontri furono orgoglio e monopolio palatino. e se dalla chinsa reggia agli aperti circhi circonfulsero in una

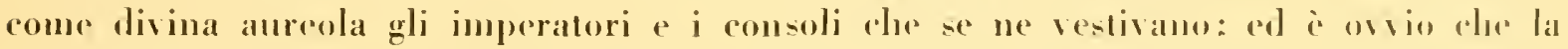
prerogativa suntuaria passasse nellevo seguente ai principi della Chiesa, ai re al duci. ai signori. ai dogi e ad ogni potente. ammaliando nei fasti religion a rivili la folla incolore. con lo sarzo degli esterni apparati. anziche illuminarla ron il hme intimo dellintelletto.

L’ insaziahile concupiscientia oculormm. gual la chiama il profeta Giovanni. per la stoffe spinse i raffinati d'un tempo a vere follie economiche e morali. di eni albuian notizia preeisa nelle storie. a l'eco poetica pur uella fialue. Velle Mille e mun notte. già di per sè stesse conserto simile aggli araheschi del piì fantastico broceato persiano,

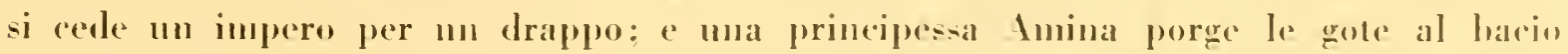
diun mercante. qual compenso richiesto fuer mal stoffa non venale di cui rasi in aghita.

Fuor diperbole. nel vero storico. non meno amate. ma per mistica rinuncia. le più preziose vesti venivano offerte dalle dame pairizic alle loro chicse per trafomarle 
ex voto in parati da usarsi ne" divini ufficii. Cosi fece Beatrice d'Este al santuario della Madonna del Monte. forse impetrando la grazia suprema d'esser resa madre: cosi si presume di altre. ove vediamo in chiese ricchezza di paramenti sfarzosi in contrasto di malcelate rabberciature.

Sotto i Papi dell"età d"oro. i broceati assursero a prezzi inauditi. certo superiori a quelli cui valgono adesso. sebbene fatti infinitamente più rari dal tempo. Leone $\mathrm{X}$ dona in appannaggio a suo fratello. che doveasi sposare Filiberta di Savoia, centomila ducati di cui trenta in vestiti. conteggiati come valuta corrente.

Cotanto estimate, le stoffe ebbero talvolta pur anche una lor propria missione, giacchè non senza intento eran le offerte di doni che accompagnavano le ambascerie famose. Per esempio quella dello sciì Abhas alla Repubblica di Venezia. resa celebre in un qnadro di Gabriele Cagliari. che ancor riflette in sintesi pittorica il sole della Persia, fu una mirabilia di colori guizzanti in tappeti e drappi innanzi al doge. E altrettanto memorabile negli annali del Micado è il ritorno degli amluasciatori giapponesi. carichi di opulenti velluti trapunti dooro. donati da Filippo II di Spagna e da Papa Sisto $T$ al lontano Imperatore. ignaro delle glorie di Aracne occidentale. Cosi una volta si raccomandavano gli interessi di stato a seducenti visioni. atte a ben disporre l'animo.

Nelle chiese. nelle reggie. sugli altari. nelle tombe. ovunque stoffe!

Al dilagare del crescente lusso. divenuto ormai una necessità dello spirito. invano si opposero le leggi suntuarie. che anzi ne anmentarono la brama. A un bando del rappresentante del governo papale in Bologna. che voleva ridotto l'uso delle troppo ricche vesti femminili. risponde Nicolosa Sanuti: "Entrare nei magisteri non si permette alle donne: non si permette loro nè il sacerdozio. nè la milizia. chè tali uffici delbbono essere virili. Ebbene: che ci siano anche tolti gli abbigliamenti, simbolo del nostro genio, a tutto potere non soffriremo ).

Simbolo del nostro genio dice dunque la Sanuti. e dice giusto: giacchè se mella molle stanchezza ereditaria delle decadenti società. affinasi vieppiù il senso dell eleganza e del colore. cui dimostrano esser meno sensibili i popoli forti, ne appar tal senso insito al sesso debole segnatamente. come hen eble ad osservare il Gozzi che ( la maggior sensihilità nellocchio delle donne le rende più atte a sentenziare della bellezza de' colori ).

E ciò posto. in aceordo con la legge darwiniana - la quale conferma esser dovuta la bellezza cromatica in certi animali a punti di approssimazione fissati dal gusto elettivo delle femine - si può per tanto indulgere alla vanità umana, cotanto censurata dai moralisti, considerandola appunto come unimmanente necessità naturale.

Dalle evocazioni storiche e generiche considerazioni. passiamo alla contemplazione diretta delle reliquie che abbiam sottocchio. lasciando che $\mathrm{i}$ loro colori morenti in sulle trame conteste di senso e di poesia ci parlino con le stesse vibrazioni di sottile estasi musicale già provata dalla squisita sensibilità di spente generazioni, e ci sugge- 
riscano la ragione logica del loro misterioso fascino.

Ci pare cosi di apprendere che in esse il piò modesto elemento grafico. insignificante a chi lo consideri dal solo aspetto oggettivo. non sia altro che una parola di nu testo musicale cui ogni nota corrisponda come al una sillaha; e nellestendere l’indagine si arriva a pensare che il disegno nelle grandi opere coloristiehe ornamentali, non sia direttamente voluto, bensi derivi dal comporre il rolore in sinfonia.

Onde certi ornati si distanti dal vero rhe neppur lo ricordano. si possono conside. rare addiritura quali residui inerti del tipico fenomeno medioevale di lenta e progressiva astrazione della forma (fenomeno che ha riscontro in quello morale delle aspirazioni ascetiche) volgente allespressione cromatica pura. raggiunta solo dall"arte islamica: la quale con i suoi geometrismi par segnare il trionfo lirico del colore. ridotto quasi in sintesi algelorica. Esagerando con l'esempio, si può dire ancora che in essa. e nella medioevale in genere, il disegno stia al colore come l’incisione fonografica alla propria emanazione acustica; e meglio infune ci possiamo convincere praticamente di questo rapporto. con l'osservare la traduzione fotografica di un liel velluto: il quale. privo in essa delle tinte. mostrerà i più suggestivi particolari ornamentali. confondersi in vicenda di oziosi pleonasmi senza vita.

Qui laargomento ci adduce alla menoria un passo di Kant, che sebhene rapido dis. solvesi nel corso dei nostri argomenti. è bene riferire perchè contrastandoli li delurida. e dice: "I colori che avvivano il disegno. appartengono all`attrattiva. e se possono render gradevole 111 oggetto alla sensazione, non lo fanno degno del bello. essendo essi quasi sempre sottoposti allesigente hellezza formale; e dove anche si può concedere allattrattiva, è sempre la forma che li nobilita ). Non cosi. a nostro arviso. i colori son veste attraente della forma: non dualismo fra questa e quelli. ma unità dignitosa che governa l’arte vera. Non mai ad una bella sculura si è desiderata la veste del colore. come non mai ad un bel dipinto la forma tattile; se non in egnal misura, i due predicati si compenetrano sempre, e dove ciò non aviene non vä arte, hensi materia e vernice. Tutt'al più possiamo ripetere col Helmholtz, ehe solo nellarte ornamentale il piacere del colore regna libero e svilıppasi dalle sue proprie leggi. Anclı nella pittura rappresentativa ciò che sulito ci tocea e diletta ì la macchio, ossia laccordo delle tinte in toni e luci. che appartiene appunto al momento primo della vera creazione estetica. e non è un posticcio attributo della forma.

Confermano in ispecie codesto asserito carattere unitario. e però lirico. gli schemi metrici desumibili dalle ormamentazioni tessili. Il solito arahesco, allontanandosi sentpre più dalla propria realistica origine vegetale. uelle ste ricorrenti volte e rivolte par talora seguire un ritmo il cui numero sia seguato dalla misurata estensione delle super. fici monocromatiche; mentre emergenti note di colore. seconlo la reciproca posizione densità e valore, rispondonsi in armonia perfetta con la rima haciata. replicata e disconante. Or mi sovviene di un damasco trecentino coperto di tralci di vite e decllera con- 
serti. i cui racemi e corimhi in alterno contrasto facevan come l'unirima d"uñingenua cantilena; al contrario, gli ornati di certi velluti gotici a rami carichi di cardi e pine. sono talvolta cosi robusti. per dire come d'Aununzio, che "eguagliano la misura dei grandi rersi memorabili ").

Per quanto sian vemuti osservando. si spiega come similitudini fra poesia e arte tessile. ricorrano frequentissime: "(il drappeggiamento del verso ". definisce il nostro Carducei; e nella remota patria della seta, la Cina (mi si perdoni il salto. ma la fantasia non conosce distanze). il pocta $W^{\top}$ ang-Ci-fu elegge con fine gusto dalle matasse seriche i suoi più leei colori. e cosi li ricama:

$$
\begin{aligned}
& \text { Con fiorito pennello } \\
& \text { dolci cose scriveste, } \\
& \text { e un drappo tesseste } \\
& \text { tessendo una canzon. }
\end{aligned}
$$

e di sè ancor dice per bocca della sua immamorata:

$$
\begin{aligned}
& \text { Alate frecce uscirono } \\
& \text { dall'arco de' suoi versi; } \\
& \text { Son le sue strofe serici } \\
& \text { drappi a color diversi. }
\end{aligned}
$$

In guisa inversa. I"arte tessile. nonchè similitudine poetica, può essere ella stessa ispirata dal canto. come appare nel seguente aneddoto.

Isidora Duncan. la danzatrice. studiosa di riposti ritmi che regolano ogni forma d’arte, non escluse le fahrili. indossando per ritorno all'Ellade - terra delle perfette arnonie - un classico peplo di lana bianca tessuto di sua mano a onde cerulee irregolari simili a yuelle del mare. venne richiesta da un amica se le ne avrebbe voluto fare un altro egnale... " ma non so - sogginnse - se il motivo mi ritornerà preciso perchè non ricordo il canto che tessendo cantavo m.

Il lavoro della tessitura. fra lo strepito delle telaia battenti. era accompaguato in altri tempi dalla gioia di lieti canti modulanti il polso della spola e del pettine: e ognopera compiasi con un sorriso di compiacenza che le raccomandava ai secoli, e che ancor rimane riflesso in esse qual suggello d'onesta bellezza: mentre le stoffe moderne echeggiano invece la blasfema dellinfernale travaglio meceanico

$$
\begin{array}{r}
\text {..... (E tre maledizion l'ordito fanno } \\
\text { Tessiam, tessiam, tessiamo }) . . .
\end{array}
$$

Ed ora. riconducendo il pensiero verso l'origine del tema, mi sembra intravedervi una legge che governi le armonie e che in quelle del colore sia più trasparente, e oserci delucidarla così :

Se nella quiete che segue i contrasti par alimentarsi la vita dell"universo (e forse l"universo stesso ne esce dal cozzo di forze antagoniste), certo dal dissidio di suoni, di 
linee e di luci si compone l'armonia dell’arte. la concordia discors. Nel minimo eerchio del nostro argomento troviamo rlue i colori simili tendono fondersi in monotonia: mentre i contrarii si repellono vilorando, per conciliarsi. Ma se il Weluer ha poluto indagare nel campo fisiologico le leggi e il numero dhe governano e regolano il mutrasto delle tinte, non si è ginnti ad intuire da quali interferenze germini il piarere che conduce alle creazioni darte. Nemmeno sappiamo quale importanza possa avere ucl. l'attività estetica dei colori leconomia chimica di quella porpora risiva, scoperta dal Boll; la quale nel sno svanire e rivelarsi secondo gli stimoli rhe riceve al fondo della camera ottiea, offende o arcareza la vista prima aneora di animare le piì elevate sfere dello spirito. Certo, per questo spiraglio sensorio chłe la vista. voglion essere vagliate le impressioni prime degne di elaborazione, poiehe solo le tinte armonizzanti a priori, e non se stonate. posson prender forma d"arte vera.

Siamo pure indotti a considerare dallo studio delle teeniche negli antichi monamenti, i più modesti artefiei della grande arte anonima medioevale. non solo poeti del colore, altresi ingenui scienziati. preconrenti la moderna pittura divisionista.

È noto in ottica: che i eolori primitivi combinati sulla retina dàno la pereezione del bianco: che accoppiati formano $i$ secontarii: che puesti ultimi, contrapposti, acpuistano maggior splendore e pureza: che i primitivi, arginati di nero o di bigio, irradiano il proprio tono eomplementare.

Orbene, della nozione empiriea di codesti sommarii principii documentano non pochi tessuti; tra i quali, meglio degli altri. un damaseo il cui fondo verde in appa. renza risulta tale dal contesto della trama azzurra col giallo dellordito rado. Similo. effetto era espressamente voluto in una speciale stoffa giapponese della Oro-Iri. che secondo il comulubo delle tinte primitive eangiava di eolore e nome. In questo genere privilegia la mia raecolta un velluto arancione la eni intensiti. che quasi direi coloricen, si deve a un sostrato di stamigna rerulea, che come per antitesi alimenta l'atrdore della sulperficie vellosa non meno inesamribile dello smeraldo del prato perenue generallo dalla terra bruna. Al contrario le stesse nozioni. applieate negaltivamente, si arvertomo dove, ad evitare i fenomeni di sintesi e di contrasto. i colori confinanti sono distanziati da un margine neutro che ne tutela il giusto valore contro il reeiproco assorbimento.

Alle provate intuizioni fisiche del colore. alte corrispondono relative alla forma. Abbiamo per esempio porpore bizantine a grandi discgni dentellati, i quali, visti in distanza, rendono meglio i profili nella loro severita che non se fosisero espressi in modo curvilineo. Il fatto si volle altribuire a ragioni enconiche. presupponendo un armalura del telaio inadeguata al passo del motivo troppo grande; ma la costanza di tal falto. anclue in tessuti più recenti e perfetti, suggerisce piuttosto una voluta sintesi lineare. ormai del resto acpuisita all"arte odierna.

Agginngiamo ancora che certe imperfozioni del discgno tesile. rerte asimmetrie. non ginstificate da diffeoltà meceaniche ei sembrano cereale apposta per rendere piò vivo e mohile leffetto del motivo, altrinenti uniforme nella sua precisa itcrazione: e de- 
vonsi forse a quel optical refinement, che in materia d'architettura medioevale è stato riconosciuto e studiato dal Goodyear come ragione di talune apparenti anomalie. quale per esempio la entasis dei templi greci.

Oltre agli accorgimenti. l'osservanza dei limiti tecnici imposti dai fini estetici condizione prima del buon gusto in ogni arte libera - rese eccellente la tessitòria antica. Uno di codesti fini pii caratteristici è di fermare tutta l'attenzione alla superficie su cui sono tracciati i disegni; incuantochè essi non pretendono rappresentare. come in pittura, le apparenze dello spazio con effetti di chiaroscuro, bensi intendono a riflettere piane architetture di tinte che ricreino locchio riposando la mente; e in questo senso accettasi la definizione pitagorica che il colore è superficie, perchè tale veramente vuol essere nelle opere ornamentali. Ad esempio. mentre un candido schermo dà libero adito alla fantasia dimmaginare ciò che dietro esso nasconda, un drappo operato. con i suoi mille arabeschi svolgentisi in ritmiche ricorrenze, intercetta lo sguardo divertendolo meglio che non possa un arazzo figurato. simulante prospettiche lontananze quasi attraverso finestra aperta su diun orizzonte paesistico o sur una storica rappresentazione. Non si pui adunque concedere grazia al recente telajo Jacquard; il quale travisa tale caratteristica di modesta dedizione alle necessità meccaniche dell"istrumento produttore, e conferisce ai suoi prodotti. con sforzo di congegni artificiosi. quegli attriluti più propri alla pittura; nè per tanto indulgere sapremmo alla traduzione arazziera delle grandi raffigurazioni raffaellesche. nè a quelle di certi artisti francesi asserviti alla Manifatura Golvelin.

Inaltra accertata osservanza pratica degli antichi tessieri si era quella di mantenere il rapporto fra i varii spartiti di pieghe e il tipo dei motivi, secondo la flessibilità e l'uso delle stoffe; sicchè le pesanti tende di velluto per padiglioni o troni. avendo ampio il portamento plastico. recano maggiore e rado il disegno, mentre minore e folto nelle leggere sete vestiarie, perchè sia patente attraverso le anfrattuosità più frequenti e complicate.

E pur dalla diversa qualità fisica della superficie tessile, or liscia nei rasi, or ruvida nei tabi, or morbida nei velluti. cangiando l'aspetto alle tinte. se ne trasse partito per la ricerca di effetti monocromici, specie nei damaschi che adombrano ornati in tralice all'oscillar delle luci de' torcieri e dei lamparlarii nelle chiese e nelle antiche sale patrizie.

Si vollero anche dalle stoffe duplici aspetti cangievoli secondo il punto di movenza, come dimostra un altro nostro velluto rosso su rosso a tronchi stilizzati da cui spiccano in oro piante di cardo divelte: le quali sembrano in distanza campeggiar sole, mentre avvicinandole ne riappare l'incerto disegno del fondo.

Tracce di simili mutevoli visioni le ritroviamo avvertite in Contemplazione della Morte dove si accenna di certi velluti « a opera. densi dina richezza profonda e diversa che si scopriva poco a poco "); e altresi in una novella di Edgard Pöe, la quale descrive una sala dalle ingenti pareti tappezzate in drappo d'oro con figure arabesche, che parte- 
ciparano al vero carattere orientale sol quando riguardate da un dato punto: "Per chi vi entrava avean l'aria di semplici ghirigori, ma nell"avanzare. la prima impressione spariva a gradi. finchè il visitatore vedeasi ad un tratto circondato da una ridda di spet. trali figure, sorte come per incanto da nu incubo angoscioso) ). E l’illusione era resa ancor piì suggestiva per un artificiosa corrente d’aria dietro le tappezzerie cluc le faceva palpitare come cosa viva.

Codesta originale invenzione del soffio animatore ci ricorda di certi temi grotteschi, che quasi emanati dalla decomposizione della forma, sembrano fatti per il movimento aereo delle danze, onde i loro smaglianti colori, nella variata e fatua successione di ombre e di luci. nello sparire e rivelarsi in halenanti flessuosità. acquistano la stessa potenza ammaliatrice della vihrante rosta del pavone. E quasi animale is la vita che attraversa la trama di certe stoffe in moto, vita che vediamo riprodotta con potenza fisionomica nelle grandi fuzioni pittoriche. dove i drappi dàno talvolta il maggior fascino e sono spesso il ricettacolo dello stile, se non il segno del hnon maestro...

... Il segno del huon maestro. perché, passando ad altro, gli artisti le anaron veramente le stoffe. Fra i tanti. lo dice nel trecento Lorenzo Veneziano con quella sua indimenticabile Incoronazione della Vergine ch’̀ tutta un loroccato. da cui sincarnano appena visibili i volti e le mani divini; lo dice nel quattrocento il Crivelli dai manti delle sue Madonne; nel cinque. la grande triade veneta: nel sei, il Gentileschi, già felicemente lefinito " il piì meraviglioso tessitore che mai alıhia lavorato fra i pittori »: lo proclama nel settecento il Tiepolo. l'agitatore titanico di drappi d'oro ai venti dell Olimpo; lo conferma infine l'intera pittura veneziana, che deve tanto alle stoffe d'oltremare la sua tavolozza sonora.

Ai nostri di ancora. prima che fossero ricercate dai collezionisti e rechnse nei musei, le troviamo profuse negli studii dei pittori, logore e sbiadite dagli anni. ma così piente a loro dim indefinita e mutevole richezza, da divenime necessaria fonte dispirazione. Cosi con lo stesso spirito onde Leonardo consigliava i discepoli a guardare nelle macchie formate dal caso per iscorgervi cose mirabili e varie, gli artisti sanuo anche meglio dalle stoffe antiche, seguendone i meandri capricciosi attraverso le pieghe mille. suscitare all'infinito le imaginazioni estetiche; le quali, se pur contrarie allesegesi ohiettiva, tingonsi talvolta di novellá poesia: onde cose una volta spregiate " inavertite. ritornano in voga per il nuovo significato sofistico che lodierno gusto sempre rimovantesi attribuisce loro.

Se merito o colpa del tempo pittoresco e mutevole dei gusti, non saprei. Certo il tempo che insensibile al genio umano. passa su le opere nostre. e tende restituirle al dominio della natura nelle specie originali tolte al seno di lei. dona pure alle stoffe quella nobiltà spirituale delle cose morenti. Chi abhia avvertito come una ruina vetusta. dalle colome momehe. dai fregi logori, dalle mura dirute ammantate diedera. partecipi quasi più al paesaggio geologico che non allarte umana, può anche in esse stof- 
fe seguire questo nostalgico passaggio dal fittizio al vero. dall'effimero all'eterno: e così gli orditi consunti. su cui i disegni della trama smarrisconsi in tenui sfumature, come le immagini precise in snllo sranire dalla menoria. ci rievocano stati d’animo che già prosammo, al variare di prati fioriti o al sussurro di aerei colori in tramonti lontani... "Les étoffes parlent una langue mnette. comme les fleurs. comme les soleils conchants ).

E poiche la rimemhranza di Baudelaire si accompagna appunto al tramonto. e le stoffe più non cantano alla luce morente. ma si raceolgono in silenzi dombra, convien richiudere le arche sul sommo dei colori. 
ORO TESTILE

E SAGGIO TECNICO-ANALITICO 



\section{ORO TESTILE E SAGGIO TECNICO-ANALITICO (*)}

Dell'oro ricamato e intessuto nelle vestimenta, fun dai tempi biblici, ricchissime si dimostrano le fonti letterarie con accenni e specifiche menzioni; e l'argomento precipuo per la storia del lusso, già possiede una sua bibliografia, in gran parte filologica. intenta a ricostruire dagli etimi la ragion tecnica delleco di tanti remoti splendori. Ma se a risparmiarci il dublioso lavoro di ipotesi. gli effmeri tessuti dai maggiori abissi della storia non sono potuti ginngere a noi nella loro integrità, non mancano tuttavia gli incorrotti avanzi tombali di oro testile, sufficienti a svelare piì sicuramente alemi segreti pratici di Penelope arcaica.

Si conosce per essi. che le tumiche dei Greci e degli Etruschi recavano lalvolta bratteole impresse a vaghi ornamenti propri al palpito dei veli. come meduse, farfalle, libellule e colombe. Sappiano altresi - e i ritrovamenti ellenistici della Crimea lo confermano - che looro non consentendo esser filato. venju battuto in sot. tili striscie laminari. onde conserto alla scta piegavasi ai vanitosi capricei di Aracne palatina, forse nella stessa tecnica primitiva d'alterno intreccio suggeritaci lalle reliquie del sepolcreto longohardo di Castel Trosino.

Se non in quale modo pratico usato, quanta la profusione d'oro nelle vesti al

(*) Da Dedalo, anno I, fascicolo II. crepuscolo dell'Impero. si può imaginare per le quaranta liblore che se ne ricavarono dalla combustione del manto che arvolgeva la salma dell’imperatrice Naria, consorte di Onorio. riesumata. pontificando Paolo HII, in occasione dei lavori alla basilica di S. Pietro (1).

Al naseente medioevo l'oro sembra divenire più docile al telaio, perchè si trovo il modo di ammentarne la resistenza avolgentolo a un'anima di cotone o d"altra materia allatta; na non ancora in questa nuova tecnica volle obbedire alle maggiori esigenze pratiche ed economiche della tessitura corrente, tanto che continuo a rimanere privilegio di pochi. massime nelle pazinnti acupitture, secondo la vecchia tradizione occidentale, resa insigne dal cosidetto " Velo di Classe ), e in tessuti propriamente delti se ne avverte la presenza al hodecimo secolo. solo in certi galloni (aurifrisia) di regia manifattura panormitana.

Nei seguenti invece va diffondendosi per i centri della civilà islamitica nn muovo genere d"oro lamellare, reso più resistente al ginoco della spola e del pettine. modiante un sostrato di membrana. Codesto oro membranateo. che ivi imanzi profuse allarte tessile facili fulgori. par sia un surrogato del papirifero cinese. che ra appunto di cartat dorata tagliata a sottili striscice comes tullavia in hroceati moderni del luowo.

Di siffatto oro papirifero - rhe non poteva esser imitato in Occidente per la 
maneanza di carta serica - abbiam potuto nondimeno constatare la presenza sporadica in qualche hroceato ispano-moresco del duecento, ritenendolo $\mathrm{m}$ prodotto d"importazione pervenuto da quelle stesse vie carovaniere attivate dal commercio romano e percorse dai mercanti di seta prima che da noi se ne conoscesse la coltura.

In Italia nel secolo decimoquarto. loro membranaceo lamellare di derivazione orientale si perfeziona in filiforme-spirale, caratterizzando in ispecie i drappi di Lncea famosi anche per esso.

La conoscenza tecnica di codesto oro testile spiega precisamente il significato della parola orpello. dal latino auri pellis, o pelle dorata. la quale dara pure il nome al filo ottenutone: "Hanno supplicato alla prefata Altezza Serenissima. che si proilbisca tale orpello tagliato e filato ad uso d'oro, acciò non se possino fare trine. nè frange nè altri lavori ") (2). Onde chiara risulta la similitudine del fiorentino Allegri: " $\mathrm{A}$. vete voi finissimi capelli che paion tanti orpelli ). Finita la produzione e l'uso di tal sorta oro. ne rimase il nome a signifieare ogni aurea apparenza. e in senso traslato eblue valore di finzione.

Liarginpello, per analogia, era dnuque filo di pelle argentata. di cui non manca la presenza in drappi del trecento. Arginpello e orpello si usavano filare in accia o sullaccia chera fil di canepa o di lino: accia alba ad filandum aurum (3). Nella nota delle spese fatte nel 1271 per un paliotto all Abazia di Westminster è ricordata loperazione del tagliare le memlırane dorate in listette da filare: " Et in operacione dicti auri. et scissura et filatura ejusdem III j l.: XIIIs). (4)
Negli antichi inventarii liturgici spesso torna menzione oro di Cipro. di Colonia e di Bologna che certamente era membra. naceo tuttiuno. salvo loorigine e la qualità, la quale si può dedurre dal prezzo dei loroc cati che lo contengono, secondo cindica un trattato del sec. $\mathrm{XV}$ su L'Arte della seta in Firenze (5).

\section{Una cappella d'imperia-}

le d'oro di Bologna . la libbra L. 40 Una cappella d'imperia-

le d'oro di Cologna. " " " 50 Una cappella d'oro di

Cipro . . . . " " "

" L'oro di Bologna riluce e fa vergogna "): ’̀ il falso che costa meno: e si riconosce ancora annerito dal tempo in tessnti andanti. Loro di Colonia. il medioere. ¿̀ forse quello un po grossetto che caratterizza i tipici stoloni renani recanti emblemi ed iscrizioni sacre in istile gotico. E il più caro. l'oro di Cipro. sidentifica per incorrotto splendore in sottili tessuti d'origine orientale.

Con lavanzare del secolo XV, l'uso dell'oro membranaceo si fa sempre più raro, e peggiorandone la qualità. cede il posto al metallico: il quale ebbe estesa applieazione nei velluti. che rarissimamente in Italia conservano oro animale. di cui invece la sopravivenza più di frequente si averte in quelli spagnuoli. fin'anche al primo seicento.

Il muovo oro metallico era ottenuto da III filo dargento dorato. schiacciato in lamina e arvolta a spira nella solita gnisa: e tale restò il tipo definitivo ancora in vita: mentre il membranaceo si smarri del tutto negli stoloni e fregi figurati fioren- 


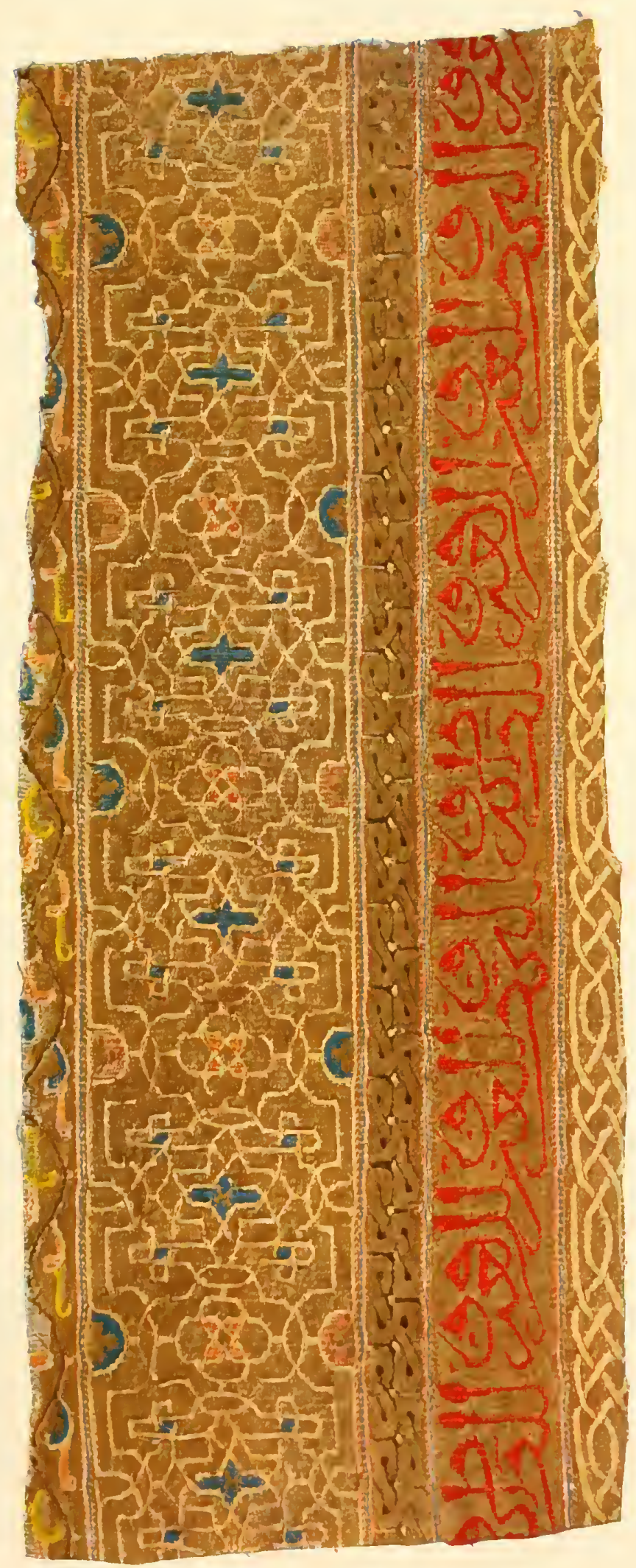





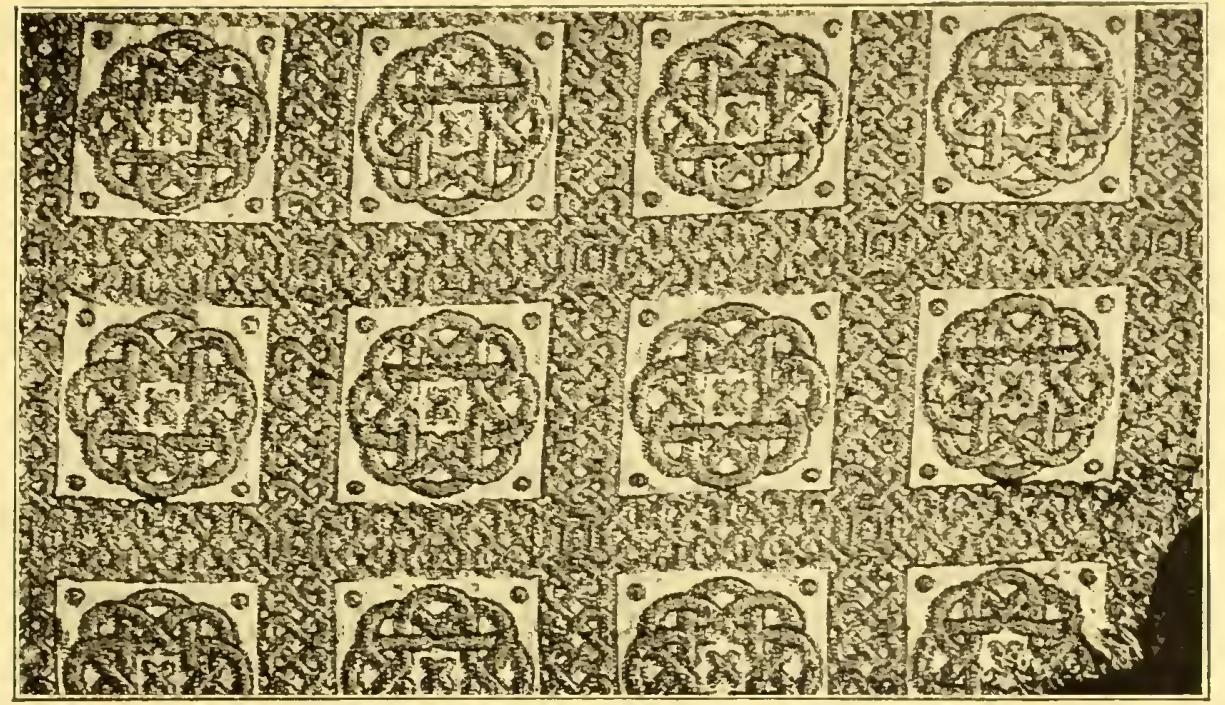

Гig. 2. - TESSITO ISPAYO-MORESCO, HBIDE

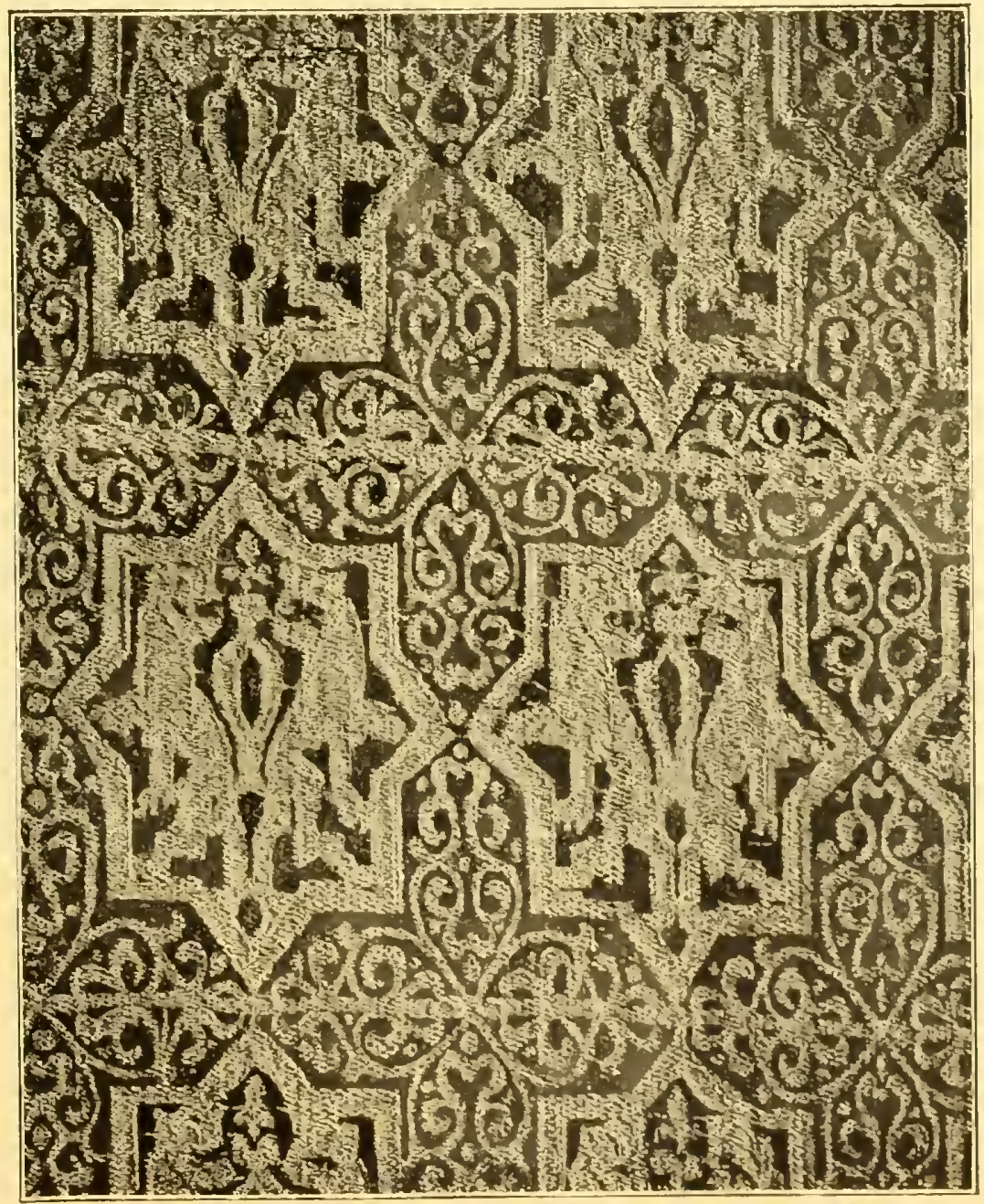


tini dell'ultima decadenza dell'Arte di Por Santa Maria. vinta dalle nuove manifatture di Venezia e Milano. più sollecite ad accogliere i benefici d’ogni innovazione.

Questi brevi cenni tecnici dimostrano quanto vantaggio possa offrire il metodo analitico, nel discernere e identificare antichi tessuti. traenti cosi spesso in inganno per estrinseche analogie dei loro ornati. Perocchè nessun'arte quanto la tessile. offre aspetti più varii ed ambigui: nè vale a differenziarli il raffronto con altre; le quali, se pur coeve e geograficamente concentriche, per ragioni specifiche di commercio. di tecnica e di tradizione. non sempre hanno lo sviluppo parallelo, ué comuni i dialetti ornamentali.

Per esempio mentre vediamo nelle basiliche romaniche pietrificati fra le volute di capitelli e fregi i paurosi sogni bestiarii di un qualche favoloso physiologus, vivace e più verosimile si muove invece la fanna nei drappi italo-orientalizzanti, quale solo in pittura la seppero ritrarre poi Paolo Uccello, Vittore Pisano e Gentile da Fabriano. Le industrie nel medioevo eran chinse come le caste sociali, e raramente alleate, anche se affini quanto la tessile e quella del ricamo; le quali. anzi. si differenziano in antagonismo pur nello svolgimento stilistico. Come può esser dunque possibile. non dico una storia, ma un singolo giudizio in materia. che si fondi sul solo raffronto formalistico. quando si verificano altresi esempi di mo. tivi stereotipi per due o tre secoli con inapprezzabili varianti? e quando tornano frequenti prove di eclettismo commerciale in prodotti di diverse manifatture concorrenti. quale la più chiara offertaci dal gruppo se- rico egizio fra l'antichità e il medioevo, riproducente modelli delle più varie origini?

Solo lo studio dei materiali e delle tecniche, applicato a quei monumenti di certa datazione ed origine, ci può fornire i caposaldi di $n$ metodo tecnico-analitico. ai quali ricorre validamente come a vere pietre di paragone. Abbandonando dunque le convenute classificazioni, mal fondate su pochi caratteri estrinseci incerti, nell'accingerci allo studio diretto dei tessuti - che direi quasi fisiologico - ravvisiamo fra di essi lesistenza di vere famiglie. classi e specie, perfettamente differenziabili per caratteri intrinseci.

A meglio illustrare codesto proposto metodo, passiamo ora ad un ordine più specifico di possibili ricerche. Nel menzionato trattato su $L^{\prime}$ Arte della Seta sono fissate le varie altezze dei teli operati, in rapporto alla qualità e il luogo doorigine loro (6): orbene, in pratica, quei dati collimano esattamente nei drappi da noi confrontati; i quali ci risultano più bassi se fiorentini. medii se veneziani. maggiori se dell'Asia Minore, e questultimi altrimenti riconoscibili da tipiche imperfezioni di tessitura. Ivi ancora nel trattato ritroviamo il tipo statuito delle cimose in rapporto al colore della stoffa (7): onde per esse si può distinguere la differente patria di certi velluti cesellati a foglie lobate e pigne fiorite, simili nel disegno tanto se spagnuoli o se italiani.

Solo quest ordine di osservazioni minime può condurre a quella sicura conoscenza della materia. che troppo spesso si vuol chianare intuitiva, e che nel caso nostro ci rende agevole il gindicare un tessuto dal rovescio e da un frammento di pochi centimetri. prima ancora di averne visto il di- 


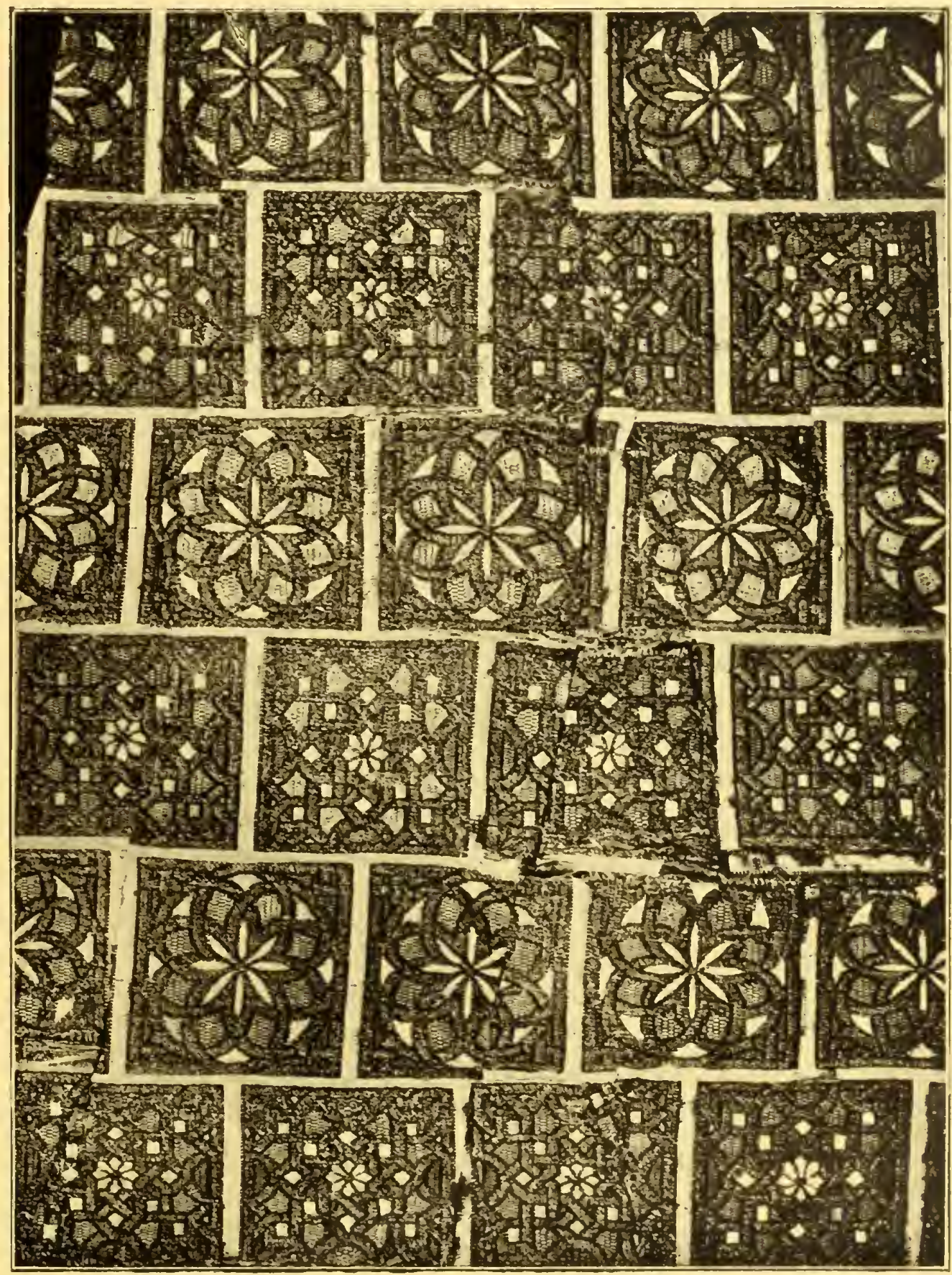

Fig. 4. - TFSSUTO ISPANO-MORESCO, IBIDFM.

segno intero. Passiamo dunque dagli spunti teorici ad un saggio pratico.

Per una fortunata occasione mi si offerse la possibilità di poter studiare ed analiz. zare direttamente alcuni frammenti del famoso paramento ispano-moresco della Cattedrale di Lérida; il quale si complone di 


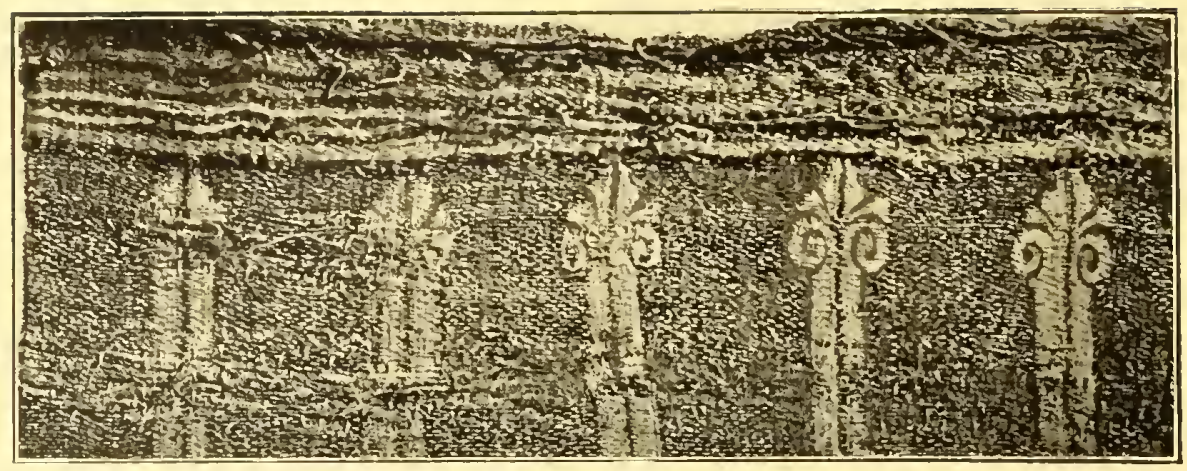

Fig, 5. - TESSUTO ISPANO-MORESCO, IBIDEM.

due dalmatiche e un piviale che figurarono all'Esposizione retrospettiva di Saragozza nel 1908 (8). e che oggi son passati al Museo di Barcellona.

Il frammento primo in importanza, ̀̀ parte di un chrysocham o fregio d'oro (fig. 1) pertinente ad una delle due menzionate dalmatiche. cncitovi insieme ad altri in guisa di tabula. sul petto. Reca una zona di intrecei geometrici in seta lianca con note di azzurro e rosa, sotto cui corre un iserizione in rossi caratteri naski. che traduce augurii di: "Felicità fortuna, grazia, forza, durevoli ") (9). Iı esso frammento si distingue il tipo dellopera dovuta al telaio verticale e analoga a quella degli arazzi coptici, la cui tecnica passò agli Arabi, e si mantenne viva nei tiraz, o manifatture di Stato; dalle quali uscirono meraviglie di pazienza e di gusto. come il celebre fregio aulico, coevo ad Haron-er-Rashid, nel Museo Araloo del Cairo (10).

Il secondo in ordine, è un campione di una stoffa celeste a scomparti quadri con rosette, intrecciati d'un cordiglio d'oro (fig. 2); della quale stoffa son fatte le due dalmatiche suddette.

Il terzo: unaltra parte delle medesime, ma iu loroccato azzurro e rosa, con quadru- pedi rampanti entro formelle stellari ( fig. 3 ).

Il quarto: un lembo del piviale, a riquadri con intrecci caleidoscopici in gem. mei colori incastonati d'oro (fig. 4).

Quinto: un rattoppo del piviale di sopra. in teletta d'oro con resti d'un iscrizione cui rimangono certi pinnacoli, che, come vedremo. son le aste maggiori di alcune lettere cufiche (fig. 5).

Quest iultimo frammento è quello che mi diè lo spunto alla precisa identificazione cronologica dell`intera serie dei paramenti leridensi, e nel seguente modo. In esso, sebbene assai mutilo, notai da prima che le parti superstiti del disegno s'identificavano al motivo di un altro maggior brano di teletta d'oro di provenienza diversa. ma di sicura datazione; il quale mostra per intero il fregio cufico, formato dalla parola Allah, replicata in doppio ordine rovescio. sopra una zona di ornati geometrici ( fig. 6). Cotesto con altri pezzi suddivisi fra varii musei e collezioni private. faceva parte del manto dell"Infante Filippo. figlio di Ferdinando il Santo e fratello di Alfonso X, morto nel 1252 e sepolto in Villalcazar di Sirga, presso Palancia (11).

Ma come la tombale reliquia, data la provenienza, si presentava meno conservata 


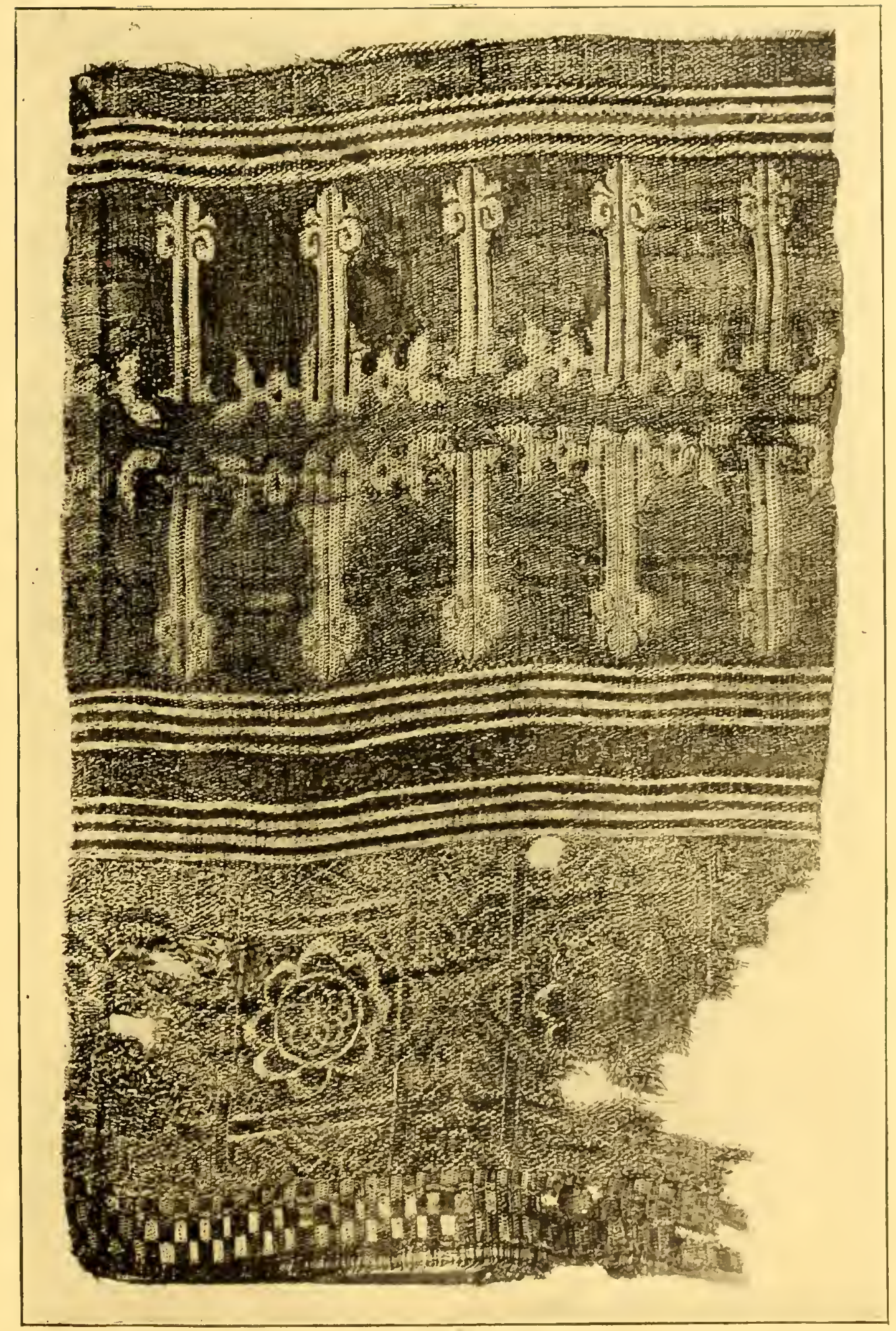

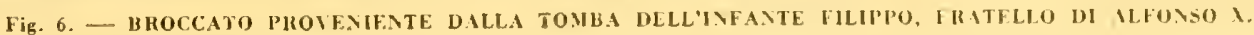


dell"altra sua simile. e l"oro n"era un po" seurito, per accertarmi di una supposta origine manifatturiera comune, volli intraprendere l'analisi comparativa delloro nei dne esemplari: dalla quale mi risultó la perfetta identità di esso. documentata da dıe fili serici, l'uno giallo e l"altro rosso. ritorti insieme e avolti di strisciette carta dorata (oro papirifero).

Cosi edotto. esteso l'esame agli altri broccati del medesimo gruppo. con mia sor-

(1) HiLSEN, Scari scnutori nel Rinascimento. in Marzocco, anno XVIII, n. 13

(2) Bandi Fiorentini, XXVIIJ.

(3) BINI, I Lucchesi a Venezia, p. 77.

(4) ROCK. Textiles Fabrics, p. XXXV.

(5) Pubblicato a cura di G. GARG1OLLl, ediz. Barbèra, 1868 , p. 100 .

(6) Cap. LXI. Uno statulo del 1514 ha pure disposizioni sull'alezza che devono avere $i$ damanchi e $i$ broccati. Vedi M. MAGISTRETTI, Due inventari del Duomo di Milano nel secolo $\mathrm{IJ}^{\circ}$, in Arch. St. Lomb. 1909, 11. 285 ।.

(7) Cap. XLIV.

(8) BERTAUX, Exposition rétrospective de Sarugosse, presa riscontrai pure in ciascumo di essi la istessa caratteristica: onde la prova irrefutalile d'un unica origine intrinsecamente accertata intorno alla metà del duecento, in cui mori il prefato Infante Filippo.

Ecco dunque come minimi lembi di remoti splendori. dispersi al vento delloblio per vie diverse, li ha potuti ricongiungere idealmente nel tempo, lo studio d'un sottil filo conduttore, restituendoli alla loro vera luce nativa.

Paris 1910, p. 171, lav, 46-47,

(9) Traduzione di Max van Berkem dall'esemplare di Lérida.

(10) ALY BEY BAHGAT, Les manufactures d'étoffes en Egypte: Communication faite a IInstitut Egyptien dans la séance du 6 Avril 1903.

(11) F. SIMON Y NIETOS, Los antiguos Campos goticos. Nadrid, 1895, p. 128, nota; R. AMADOR DE LOS RIOS, Restos del traje del Infante D. Felipe, in: Museo Español de Antiguedades, T. IX, 1. 104 e segg. La parte uaggiore del maulo si trova nel Museo Archeologico di Madrid. La sua analogia estrinseca con le stoffe di Lérilla è stala anche avvertita dal Berlaux, Op. cit. p. 171. 
LE STOFFE E LE VESTI TOMBALI

DI CANGRANDE $I^{\circ}$ DELLA SCALA 



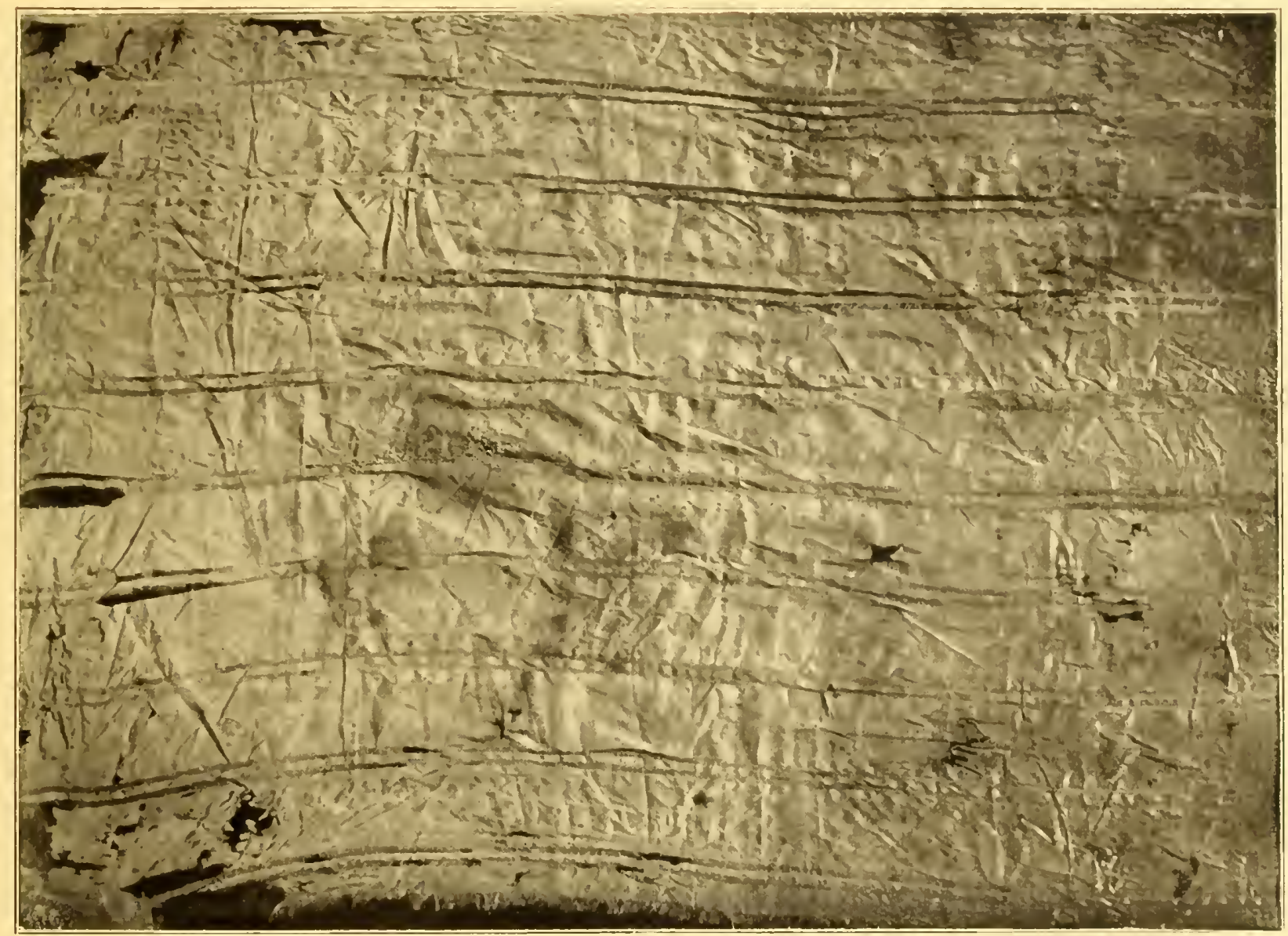

Fig. 1, - VELO SLL VOLTO DI CAVGRAXDE

\section{LE STOFFE E LE VESTI TOMBALI DI CANGRANDE I" DELIA SCALA(*)}

Ricorrendo il secentenario dantescos, il di ventisette del passato luglio aprivasi in Verona, nella Chiesa di Santa Maria Anticar la tomba di Cangrande lo della Seala. nella lavita ardita speranza di rinvenimi III qualclue ricorde che al culto di Dante si riferise (1). La speranza fu vana, ma in compenso si poterono riconosere insirme alla salma mummificata aleune lielle stoffe

(*) Dal Bollettino d'Arte del Ministero della P. I., Aprile, 1922. vive e palpitanti. che doposenoli di asemro silenzio, ai tempi del Pocta ci ridestano com flehili note di colore espuilli d"oro.

Vel loro complesso, quali vidi provitsoriamente serorinate nel Museo Civien di Terona rhe le custodisce. si presentano in honona parte immoni dalle insidie del tempo. e qualche tratlo di csese sembra romes appena swolto dalle pezze diun a mertante di gran Iraffice dopere di drapperie "), presente il signore del luogo.

lid ora, fuori dogni estrinsera conside- 


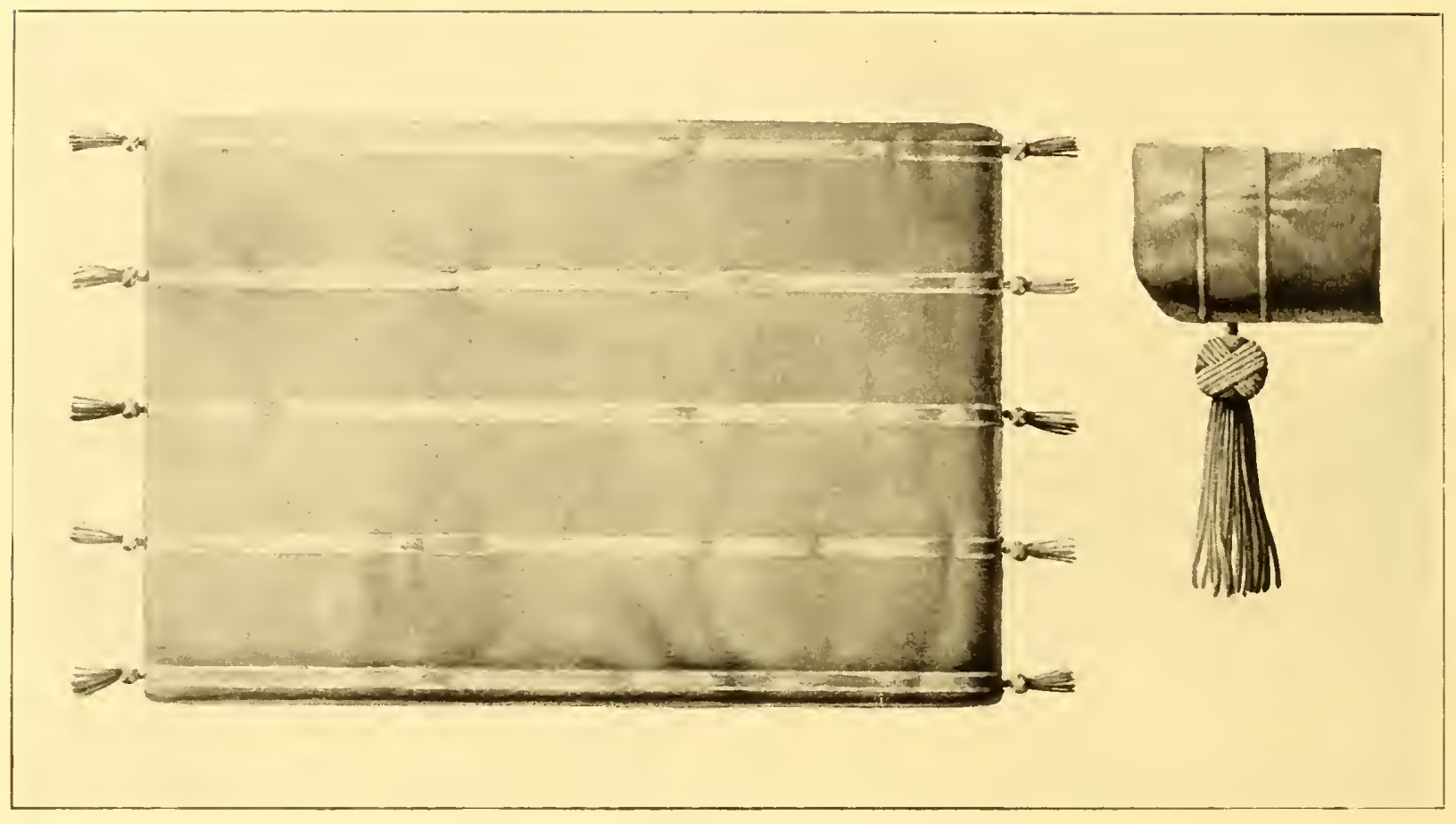

Fig. 2. - LORICIIFRE DI CA.TGR.A.DE (in pristinum).

razione, salutiamo il felice ritrovamento col darne precisa notizia. giusto $u$ incarico rieevuto dal Ministero della Pubblica Istruzione.

Undici i tipi tessili che abbiam potuto individuare, di cui quattro precipui, ed eccone intanto un puro elenco preliminare:

A) Telo di broccato d'oro con iscrizioni arabe (2), diviso in tre parti: alla maggiore, rettangolare ( $\mathrm{m} .1 .10 \times 0.80$ ), eran conginnti in origine, al lato destro, due minori lembi. formanti insieme il velo fur nelore, esteso circa quanto il vano dell'arca (fig. 3).

Le iscrizioni che lo percorrono in fregi paralleli ripetono una breve frase in bel carattere naski. il cui preciso significato sfugge agli esperti di lingua araba per essere storpiata o corrotta, sebbene ne re- -stino chiari alcuni gruppi di lettere che farebbero pensare ad una delle solite eulogie (3). A codesti fregi inscritti si avvicendano altri minori, ancli essi in seta gialla e oro, scompartiti in formelle alterne distinte da palmette e reticoli a dischi intersecati; e in sottordine fra gli uni e gli altri sono intercalati doppi margini azzurri lumeggiati di bianco a spartiti geometrici con draghi e coppie di anitre, fra rosette.

Alla testata del telo in sul vivagno, o radore, si legge impresso il mutilo monogramma:

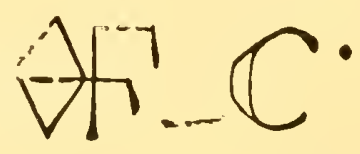

Il quale non ci sembra una marca di fabbrica - chè come tale sareblye intessuta - bensi forse un segno di pertinenza degli 


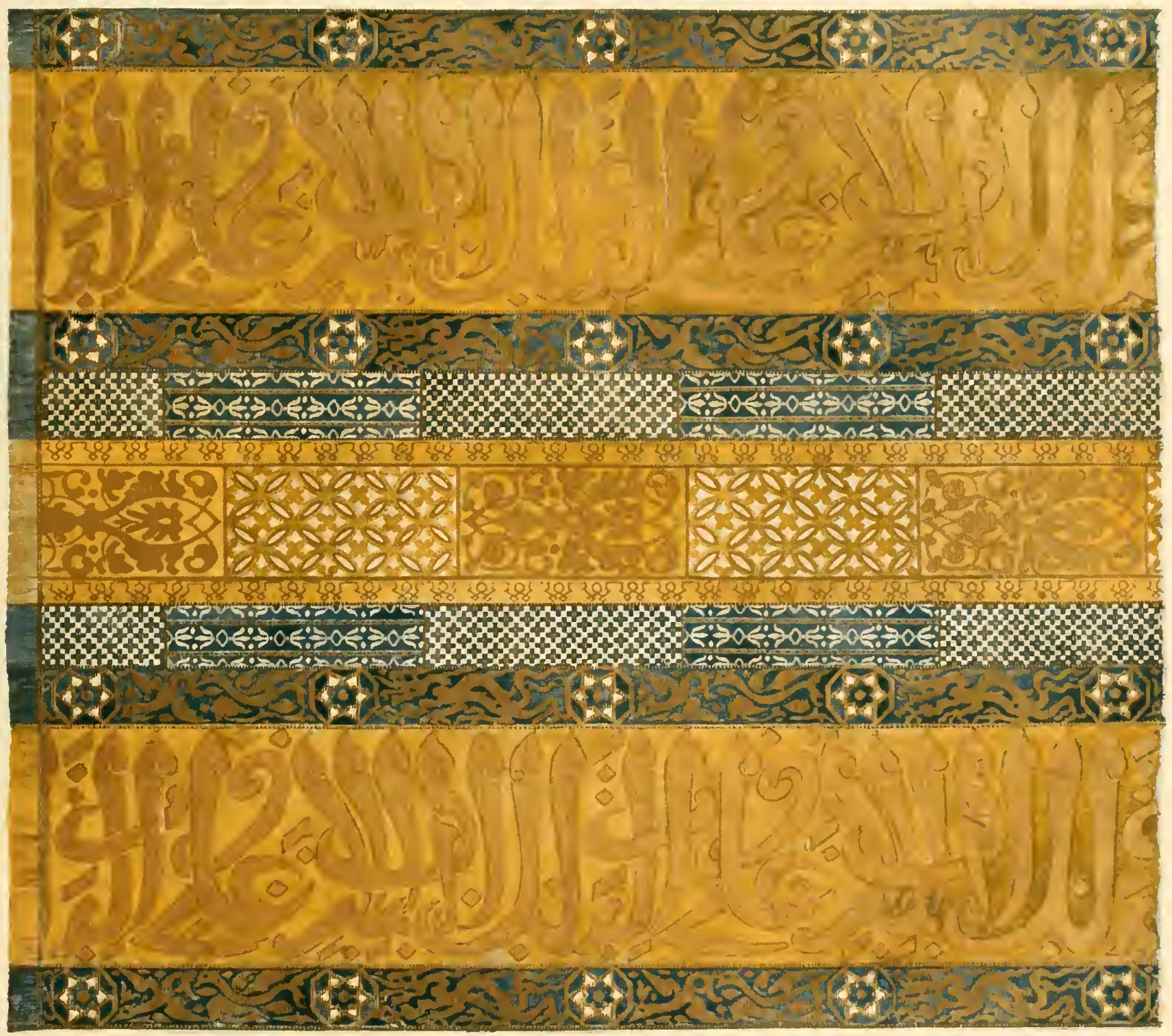





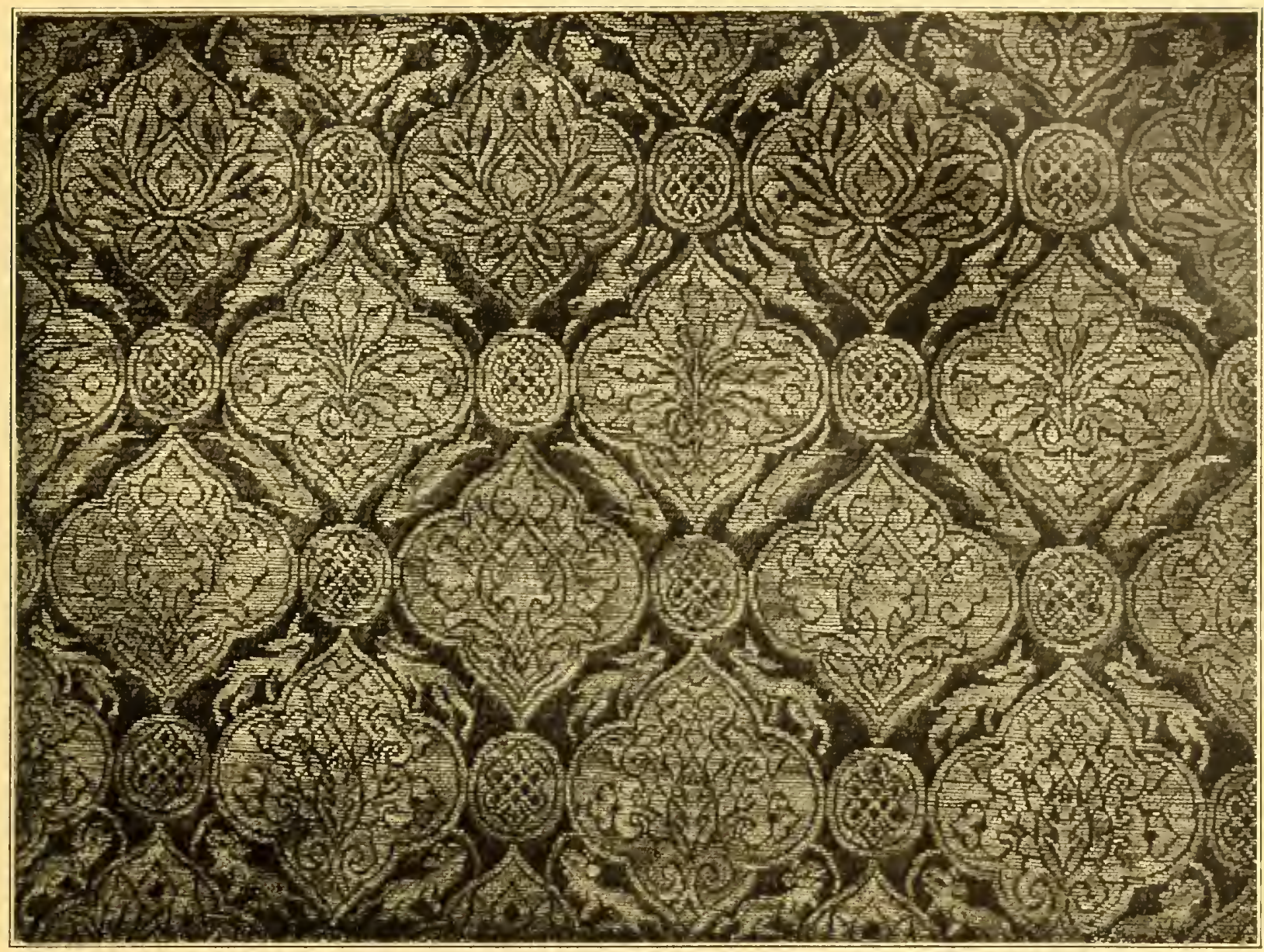

Fig. 4. - IL BROCCATO IN ORO E AIGENTO SE CUI GIACEA LA SILMA.

Scaligeri o più probabilmente del mereante che vendette la pezza.

I tre frammenti, ben conservati al mezzo, lanno i margini laceri e lisj.

Tecnica: oro laminare membranaceo: cimose gialle.

B) Velo di taffetà giallastro a doppie righe d'argento $(\mathrm{m} .1,10 \times 0.80)$, che secondo il verbale, quale sudario, copriva il volto alla salma (fig. 1).

Teenica: argento filiforme membranaceo, annerito.

C) Origliere di taffetà verde-chiaro a liste rosa marginate d'argento, e desinenti ai lati brevi in cinque nappette isocrome (m. $0.44 \times 0.30)$. Inbottito di piume e in parte consmo. Tenne trovato rimosso ai piedi della salma ( fig. 2).

Tecnica: argento filiforme membranaceo, ammerito.

D) Telo d'un broceato verde a formelle romboidali appena lohate. eontenenti quattro tipi di palmette d'oro in eampo argento, disposte in serie orizzontali, e congiunte da scutoli a intrecei geometrici. fra pesci, lepri. uceelli e leoni imnanzi una mezza luna, ehe eolnano gli spazi marginali (n. $2 \times 0,72$ ). 11 telo, trovato disteso al 


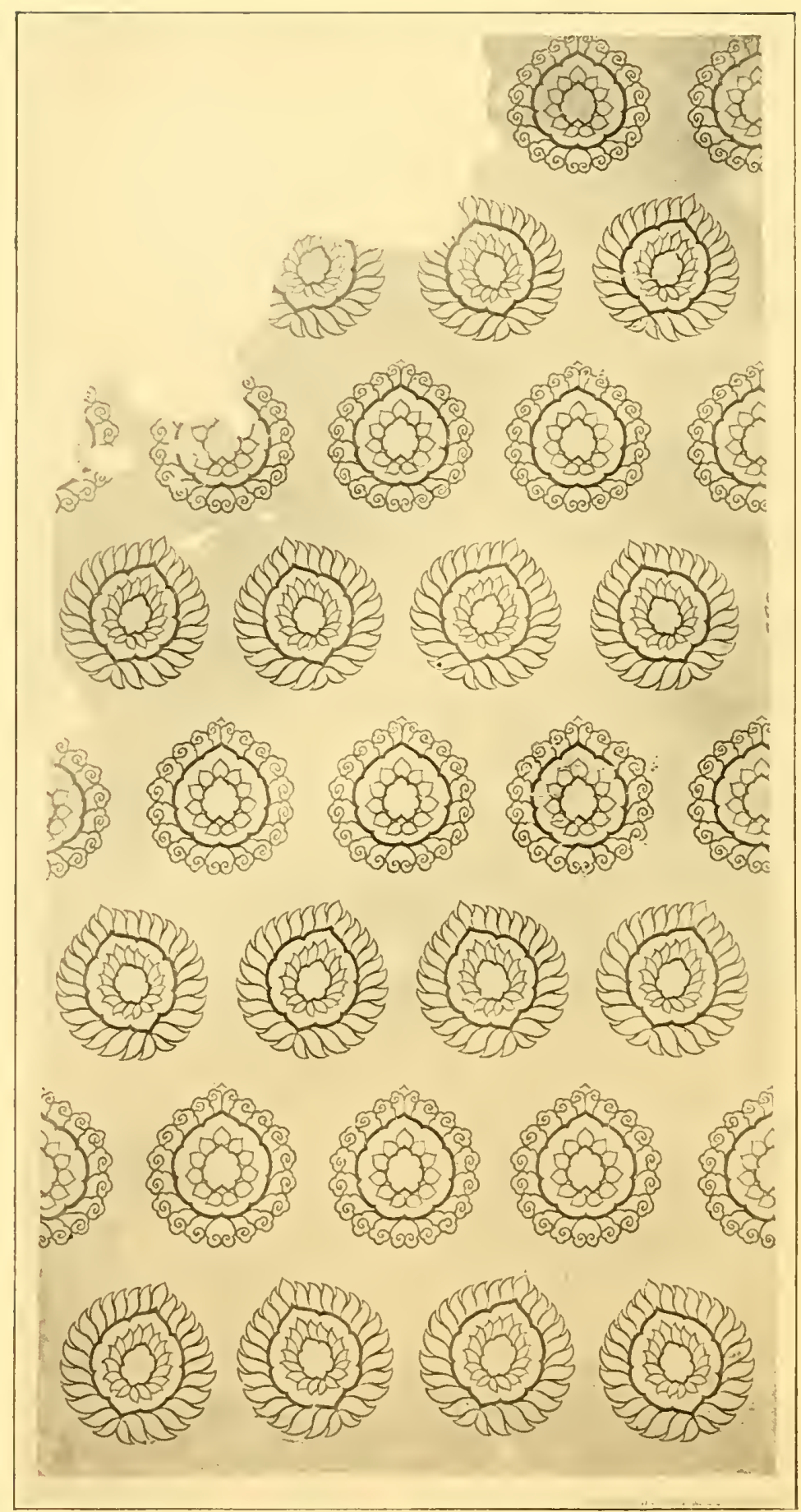

Fig. 5. - RHCOSTRLZIONE DI UN MOTINo IESTIARIO.

fondo dell'arca, mostra il tembo inferiore tagliato a mezzo. poscia sgarrato a sghtembo. Ottima conservazione; qualehe chiazza d"u. midità. e foro al punto di contatto eol pomo ferreo della spada (fig. 4).

Nella stessa stoffa: due altri pezzi gemelli, 


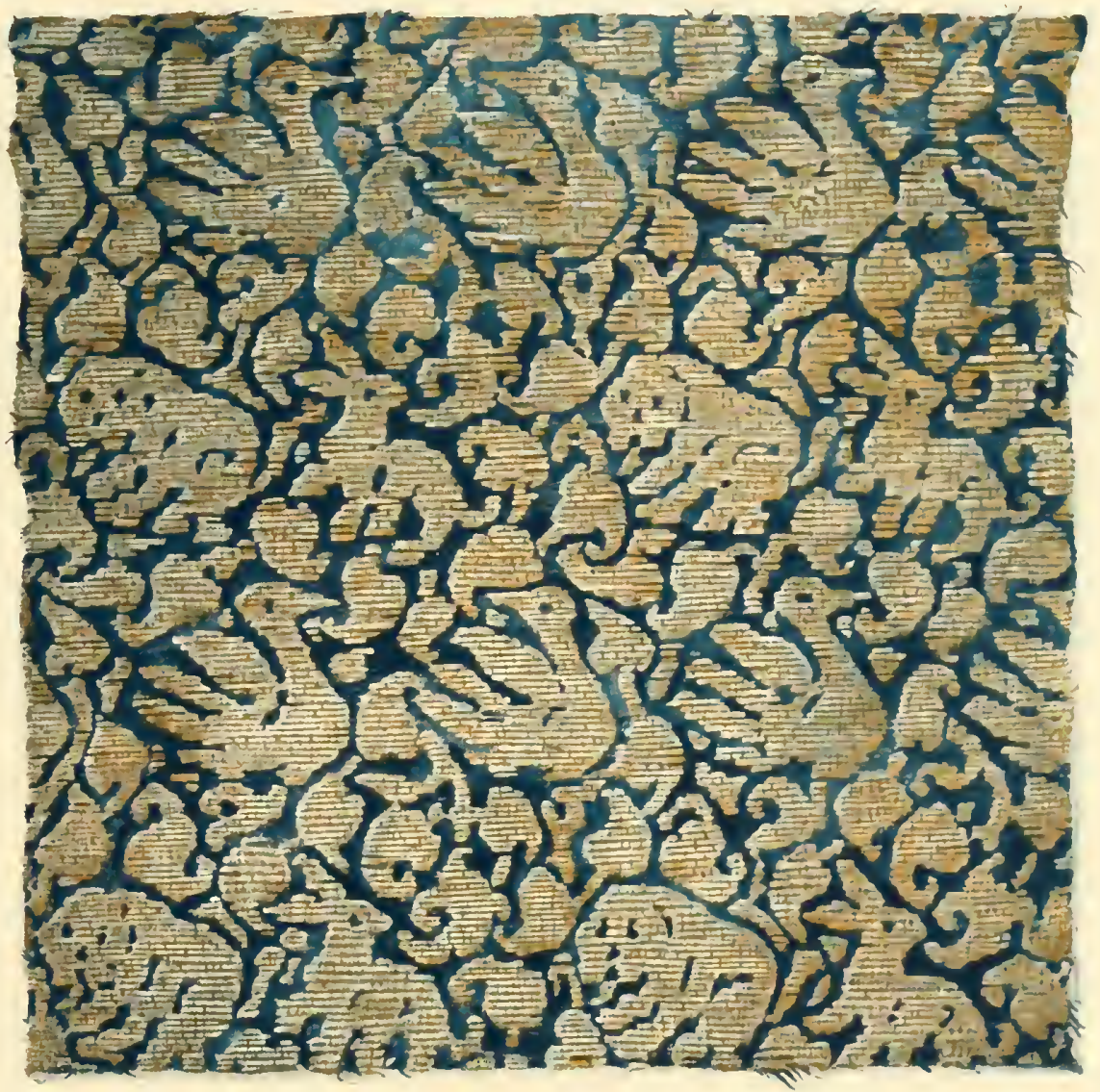

FIE - IL BROCCATC DELLA TUNCA

DI CANGRANDE ( N PRIS INUM) 


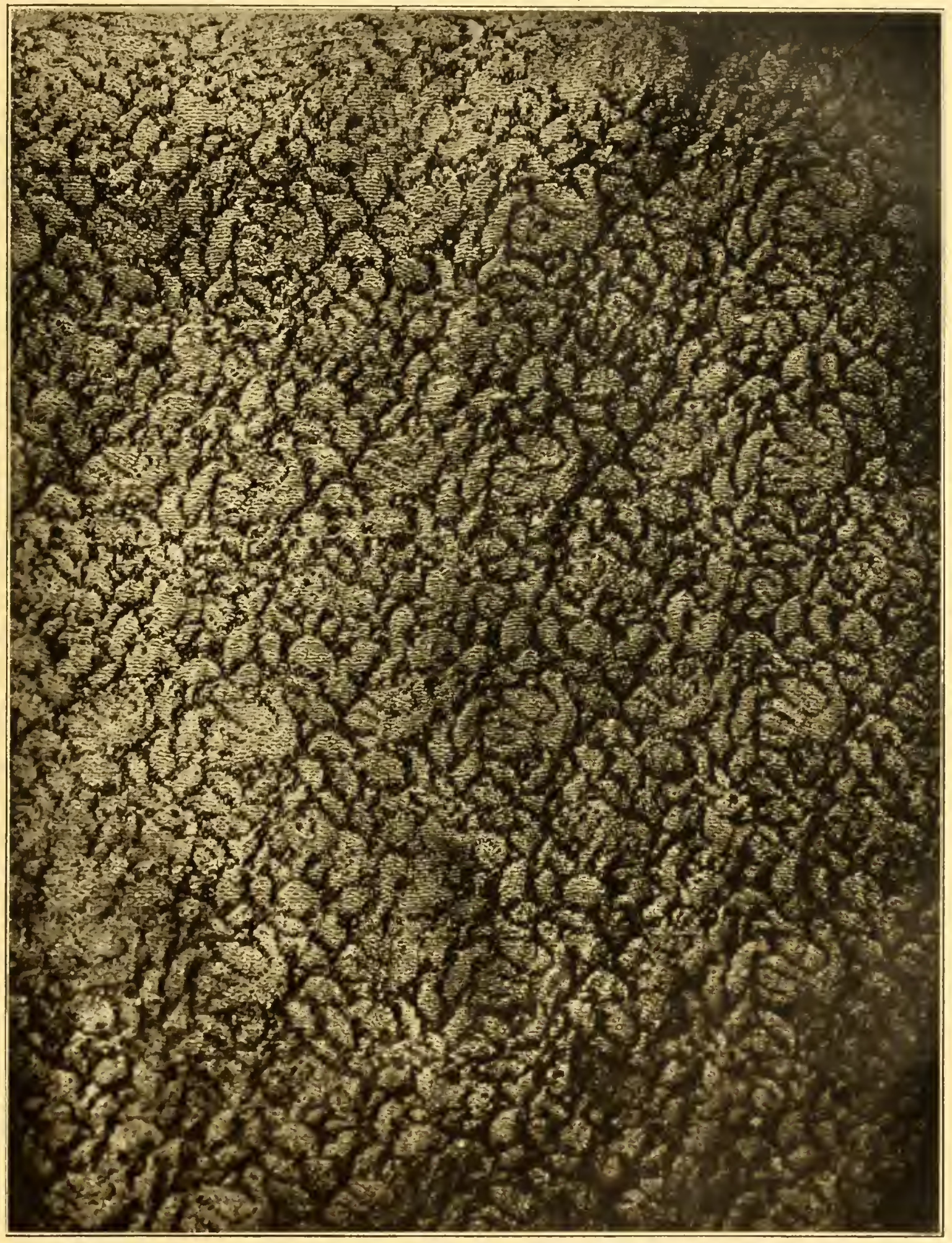


dal profilo mistilineo, pertinenti al vestiario. La loro peggior conservazione altera notevolmente l"aspetto al tessuto, che privo della parte argentea corrotta appare anche più leggero.

Tecnica: oro e argento laminari membranacei; cimose gialle ribadite da un lato con segni di scucitura.

E) Telo d'un broccato blu-cupo la cui opera in oro è quasi scomparsa e annerita nei residui, onde appena se ne legge in tralice il motivo di pigne profilate da foglioline di tipo lobato e lanceolato, disposte in quincunce (fig. 5). Il telo allato destro è privo di cimosa, e alla parte superiore lacero in guisa da far supporre una maggior lunghezza originaria (m. $0,94 \times 0,48)$.

Tecnica: oro laminare membranaceo, del quale riscontrasi la presenza allo stato pristino in un piccolo brandello preservato, or confuso con la stoffa seguente. Cimose gialle.

F) Elementi dodici di una tunica, integri e frammentarii. in broccato d'oro a piccolo disegno di cigni natanti verso destra, e di leoni e conigli alterni, gradienti in senso opposto, fra foglioline sparse in campo blu-cupo (fig. 6). Conservazione mediocre, salvo in alcuni lirani ove l'oro soprav. vive al disegno incerto.

Tecnica: oro laminare membranaceo; cimose gialle.

G) Telo di una seta crocea ad opera d'argento simile alla precedente, ma col disegno in minor proporzione e meglio perspicuo, sebben annerito nelle parti metalliche (m. 1 x 0,55). Nella stessa stoffa due tasselli uguali ( fig. 7).

Tecnica: argento laminare membranaceo; cimose gialle d"amho i lati.

II) Elementi nove di nna soprarveste, integri e frammentarii, in seta rosso sanguigno broccata a pigne in rilievo, con inscrittivi boccioli di loto, disposti fra minuscoli ornati vegetali, in campo granito d'oro ( fig. 8). Conservazione perfetta nel breve tratto riprodotto; per il resto l'oro cade in particole carboniose.

Tecnica: oro laminare membranaceo.

I) Calzari in panno rosso mattone. dal piede a punta. lunghi fino al ginocchio. Rotti in più pezzi.

L) Due tasselli di tela. ritagliati dalle fasciature avvolgenti la salma: uno damascato a piccoli rombi come le vecchie tovaglie calabresi. l'altro inadorno.

M) Velluto chermisi sul fodero della spada; la quale è munita di un passamano in cotone verde che l'assicurava alla vita. Particolare curiosità tecnica: la presenza d'un galloncino d'oro metallico lungo la costa dalla guaina. il quale stabilisce un termine a quo assai remoto per l'esistenza di questa qualità d'oro, rarissima alla fine del trecento, e già comme al secolo seguente.

Prime intrinseche caratteristiche commui alle descritte stoffe ormamentali (escluse le secondarie B. C. I. L. M.), sono la tecnica e il tipo unico dell'oro laminare membranaceo, - quasi fosse stato srolto da una singola spola - nonchè le cimose similiari clıe determinano le corrispondenti altezze dei teli. Caratteristiche queste clie affermano oltre la provenienza storica una altresi comune origine manifatturiera; la quale proveremo individuare, prendendo in esame primieramente quell'esemplare clıe più ricco si dimostra di caratteri stilistici, cioè il broceato A.

Nel suo immediato aspetto sintetico si 


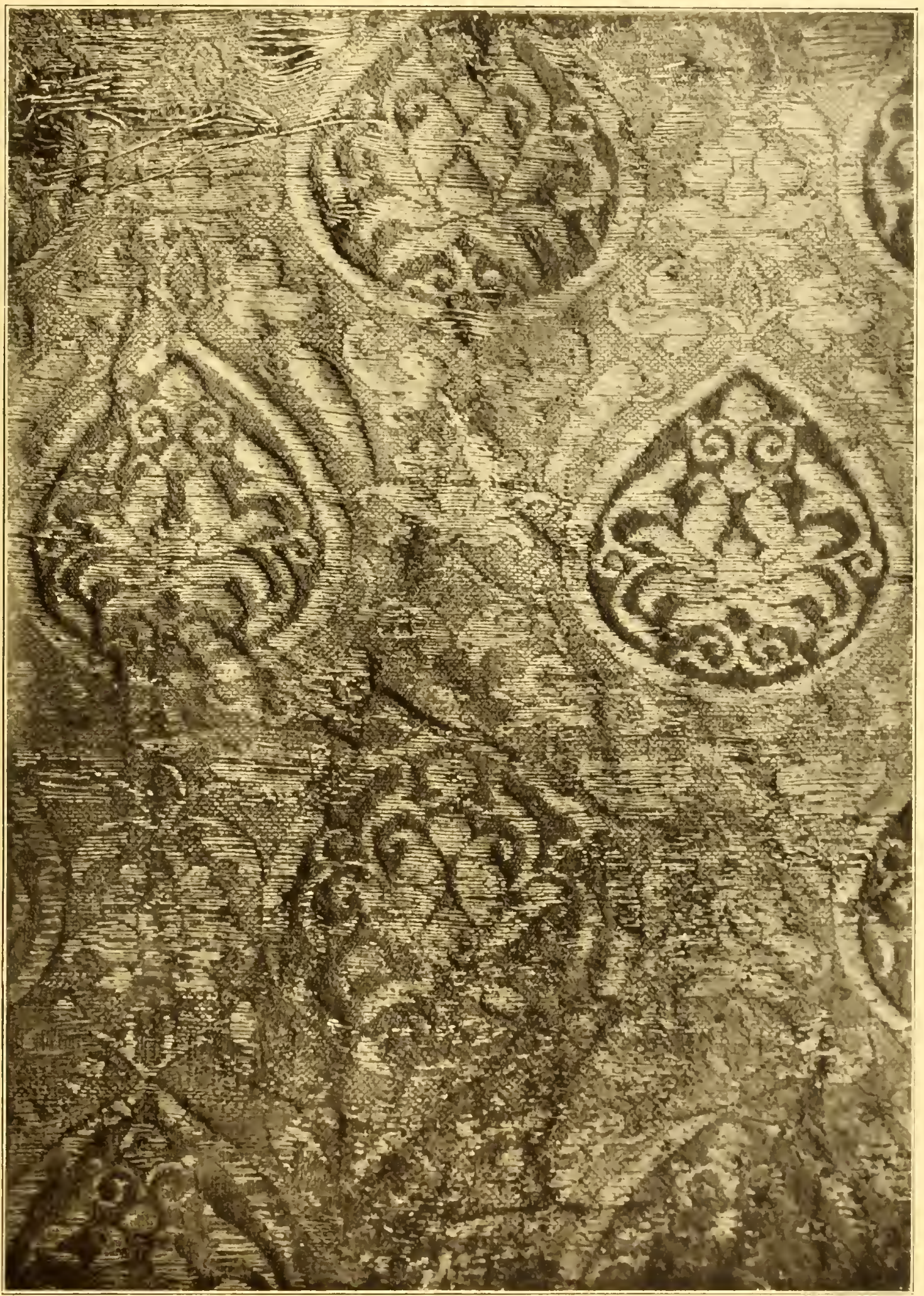

Fig. 8. - II. BKOCCATO ROSO-F NGUIGNO E ORO DELLA SOPRAWINTE DI CMGRANDE 


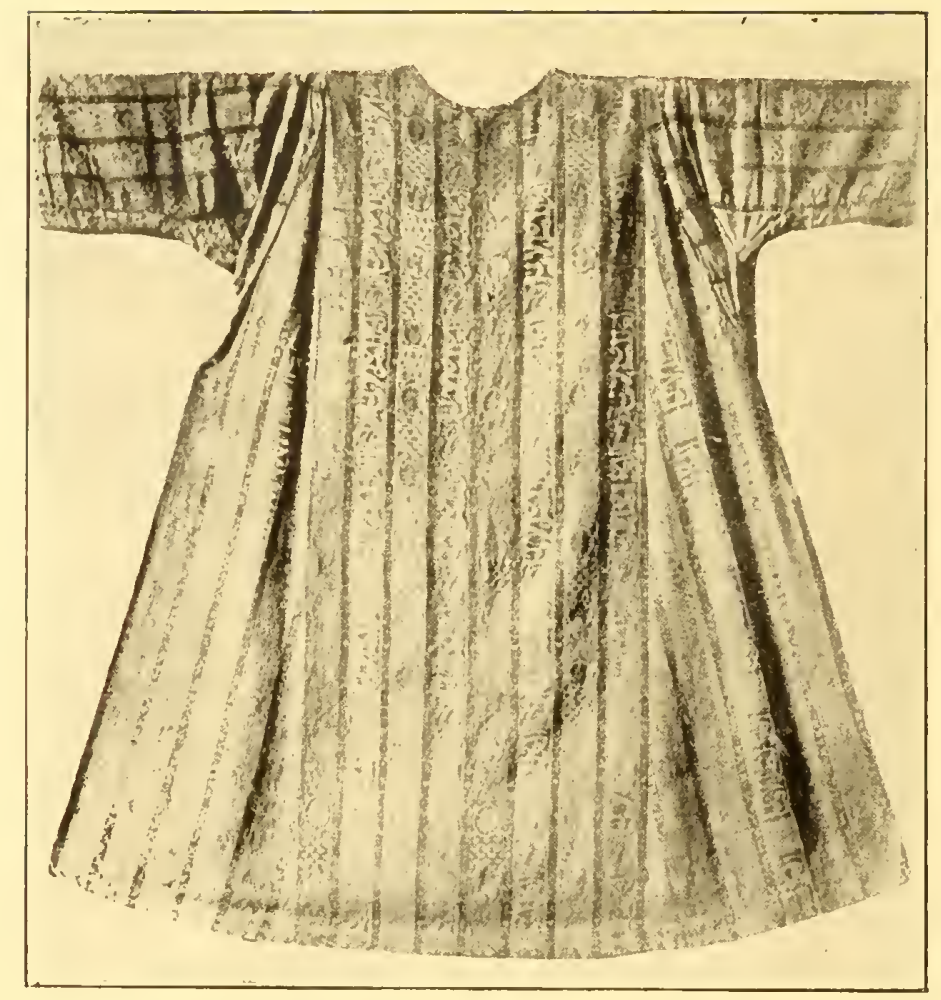

Fig. 9. - TONaCElla NElla CATTEDRALE Di RATisboNa (dal Falke).

impongono i fregi d'oro listati d'azzurro, che secondo l'inventario della Regia Chiesa di S. Nicola di Bari, rogato l’anno 1362 (4), par siano uno dei motivi geometrici più in voga allora: ivi su 430 paramenti elencati i due terzi risultano cum listis; e con liste rispondono i veli delle madonne trecentesclie.

Riguardo allo stile particolare, i motivi di detto lroceato rivelano due distinte originazioni etniche: gli mi si riferiscono al mondo islamitico, quali le iscrizioni e le palmente rifiorite; gli altri alla Cina, quali i draghi, le coppie di anitre e il reticolo, che vuolsi derivato dal simbolo budkaico ts’ien. La coesistenza di simili elementi eterogenei non appar nuova in quest'epoca; e fin dal nascente medioevo, fra le porpore egizio-bizantine già si avverte l’influsso estre- mo-asiatico (5), che poi al trecento diverrà addirittura prevalente.

Codesto fenomeno d'ilridismo par conseguire ad un arvenimento politico lontano, cioè alla egemonia mongolica, per la yuale l' impero fondato da Genghis Khan sestese ad abbracciare, oltre alla Cina. la Turchia Uiguriana, il Turchestan, la Persia con le regioni limitrofe, e parte della Russia e dell’Ungheria. minaeciando la stessa Europa oceidentale [1258-1369]. In simili rivolgimenti la funanza fu decimata. e l'emissione di carta-moneta senza valore agevolo l'esportazione dei prodotti industriali. fra cui specialnente le stoffe. contese fra i commercianti arahi e italiani ivi accorsi a far bottino.

Nel 1337 la sola casa Salinbeni di Siena aequistava in Pisa da un solito mereante di 


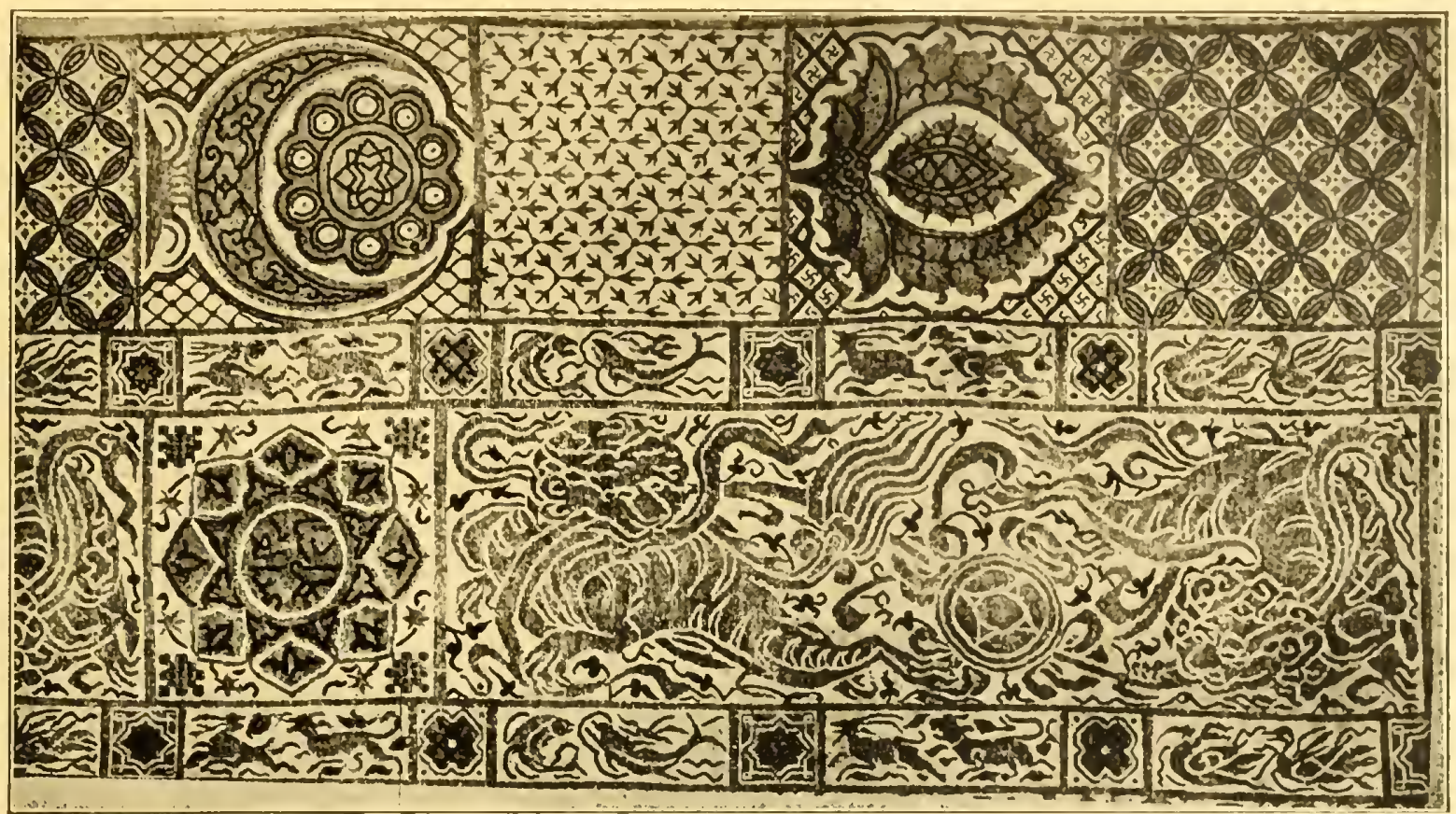

Fig. 10. - PAHTICOLARE D1 UNALTKA TOVACELA NeliA CATTEDRALE DI RATISBONA

Soria per centoquindici mila fiorini - circa due milioni e mezzo di nostra moneta seiamiti orientali di tutti i colori, panni di seta, doro, eccelera; e dentro quellanno fu tutto pressocche venduto (6). Fra i superstiti saggi di codesti prodotti d’importazione va menzionala per esempio la dalmaliea dell’incoronazione di Carlo IV 1346 nel Tesoro di Vienna, elıe si distingue per i motivi pretlamente cinesi (7).

Ma non solo le opere, bensi le maestranze stesse, dovettero emigrare in cerea di lavoro più rimunerativo, contribuendo eosi alla diffusione dellarte loro oltre la Muraglia. fino alle coste mediterance, e promovendone il gusto, per il tranite di Tenezia. nell'Italia superiore e centrale; dove le stoffe importate e imitate escritarono con i loro nuovi disegni zoografiei, pieni d'ineonsueta vivacita. un fascino profondo fra gli artisti, mortificati nella tradizione romanica. Rias.

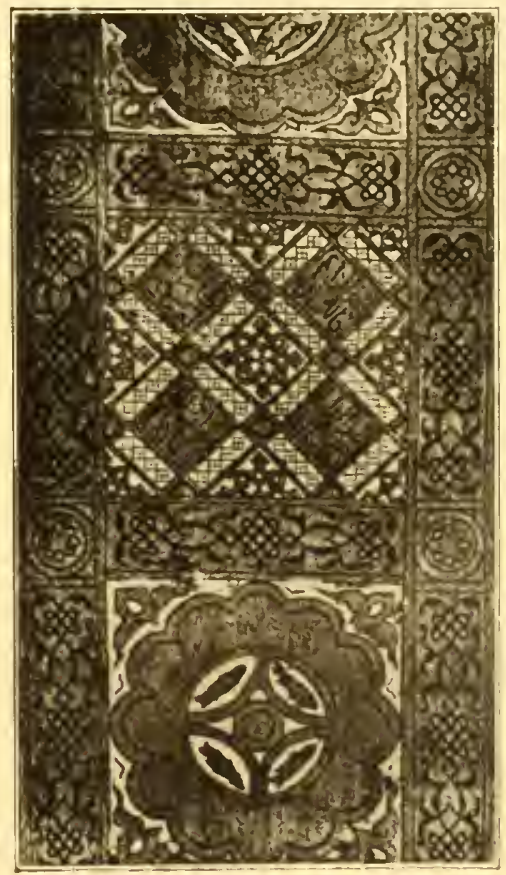

[IIIDE I].

sumendo. si puo asserire senzal esagerazione, che si elohe dalloriente islanico a noi una vera chineiserie primitiva quale quat- 
tro secoli dopo in tutta Europa (8).

Ciò premesso. la nostra stoffa scaligera risente per chiari segni codesta corrente d'esotismo, e per tanto va inquadrata in un gruppo tessile ben definito in caratteri islamo-cinesi; nel quale si ammoverano fra le edizioni principe. le tonacelle cosidette di Guglielmo II di Sicilia. nella Cattedrale di Ratisbona ( figg. 9 e 10 ). e la casula del Museo di Brunswick. il cui drappo ancor più si approssima al nostro. massime nei fregi azzurri ( $f$ g. 11). Nella testè aumentata famiglia tessile, oltre ai comuni tratti fisionomici. notiamo perfetta consanguineità nella tecnica. nella qualità dell oro e nelle cimose isocrome.

Questa determinazione di affinità ci conduce di fronte a due tesi divergenii nella storia delle stoffe: la vecchia siciliana (Fisehbach. Cole. Kendrick). e la mova cinese, propugnata dal Falke (9). La prima muove dalla falsa versione Karabacek di una scritta araha nella tonacella secouda Ratishona, alla quale si fece dire nientemeno: "Questo indumento da festa venne fatto nellofficina del maestro Abdul Aziz per Guglielmo II [ $†$ 1189] ). Laonde la tendenza ad arcaicizzare i tessuti di codesto tipo, in relazione alle fittizie caratteristiche panormitane dedotte passivamente fino ad oggi dal menzionato monumento.

Dalla demolizione della tesi siciliana sorge la seconda, cosi posta in breve. Constatata la presenza di elementi estremo-orientali, e data -- ma non riconosciuta, come vedremo - l'origine cinese delloro membranaceo, si attribuisce alla Cina stessa la manifattura del suddetto gruppo, ritenendovi le iscrizioni arabe come interpolate a scopo di esportazione.
Noi. tra le due vedute estreme ci atterremo ad una media probabilità, suggeritaci da quanto verremo esponendo. Intanto circa l'asserita origine cinese dell'oro membranaceo devesi subito rettificare - e questo ha importanza fondamentale - chessa specie d'oro corrisponde altrimenti ad un surrogato arabo-occidentale di quello papirifero, che è bensi proprio alla Cina dai tempi più remoti fino ad oggi (10); e ciò premesso. prescindendo da altre considerazioni tecniche. a stabilire se l'ibridismo in questione si debba a un fenomeno di osmosi o di endosmosi, o, in più chiari termini, se corleste stoffe siano cino-islamizzanti o islamo-cinesizzanti ci aiuta la stessa seconda tonacella Ratisbona, che piì modestamente or si riduce a dire. per bocca di esperti orientalisti. che ne intrapresero la lezione definitiva, esser " opera del maestro Abdul Aziz ): il quale maestro, come suggerisce il nome, non credo voglia passare per cinese.

A questo proposito non vale opporre che artefici e commercianti arabi ve n'erano in gran numero a Karakorum, sede del Gran Klian mongolo. dai tempi di Genghis fmo a Timur. giacchè per converso nello stesso periodo maestranze cinesi emigrarono verso occidente; e ci risulta, ad esempio. che Hulagu Khan nel 1266 trasferì in Persia un centinaio di famiglie artigiane arl esercitarvi le arti loro (11). Comunque gli seambi commerciali fra i due paesi furono sempre attivissimi, e talvolta accompagnati da omaggi lessili fra Gran Cani e Califfi (12).

Del resto. senzaltri sussidii storici, possiamo constatare noi stessi in più prolanti documenti dellarte persiana stessa, nonchè l’influsso, linfiltrazione continua 


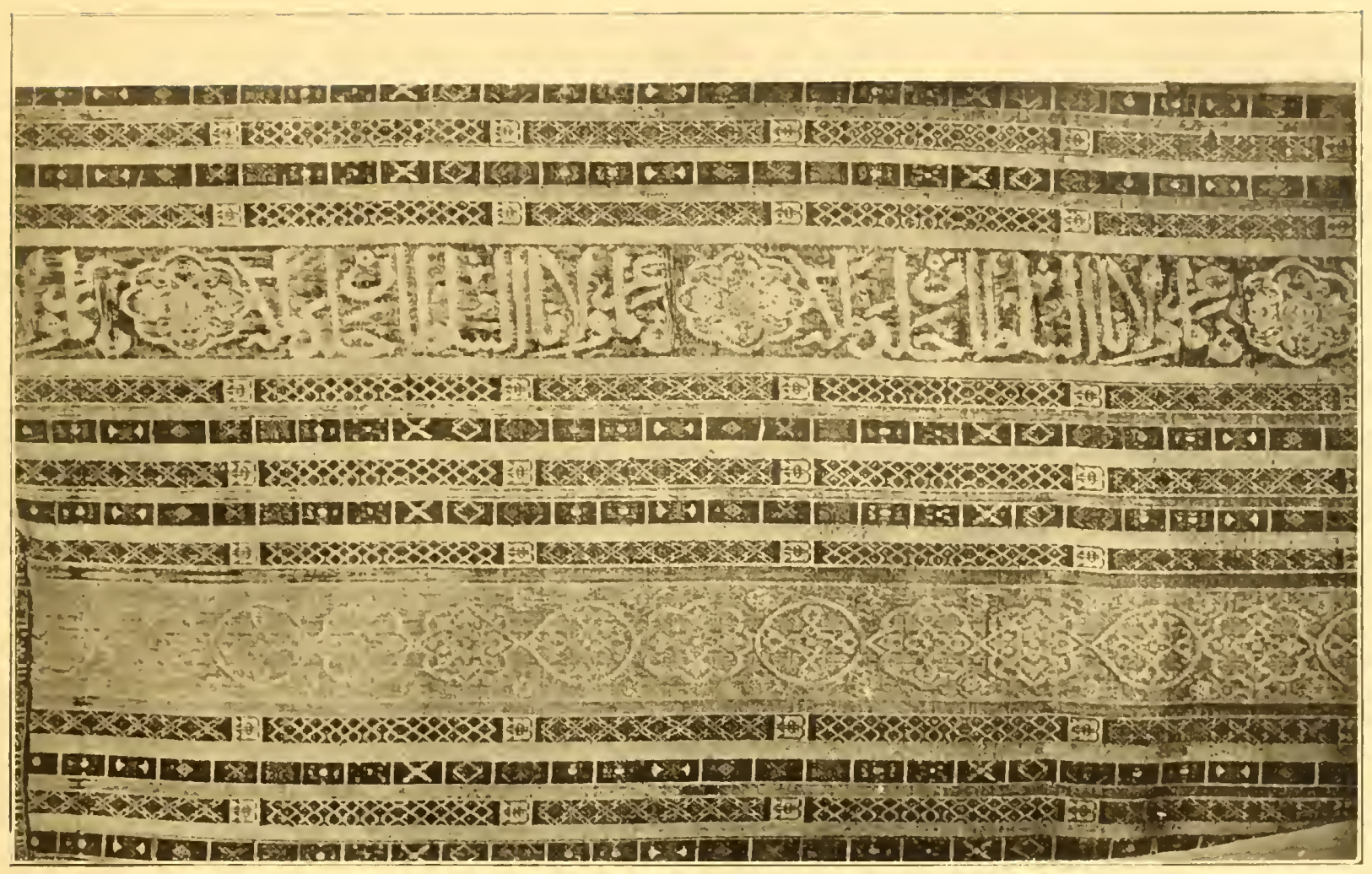

Fig. 11. - BROCCATO NEL MLEO DI BRLISAICK (dal Fathe)

di elementi serici, sia nei tappeti - che rispecchiano draghi più o meno imperiali. e nubi stilizzate a nastri - sia in agemine, sia in miniature. e sia infine in decorazioni murali, fra cui tipiche quelle della moschea principe di Varamin [1322-1412], ricche di motivi floreali cinesi distinti fra altra specie d'ornati indigeni (13).

Altrimenti. non volendo ancora escludere del tutto la tesi falkiana, mi son posto in confronto il seguente quesito negativo. Stabilito che il dritto del velo funebre di Cangrande, da cui abbiam preso le mosse, sia in rapporto alla direzione orizzontale delle proprie iscrizioni arabe, come mai gli ornati intermedii ad esse corrono in senso verticale?

Si dovrebbe rispondere, ammettendo per ipotesi provvisoria fosse lamtore del tessuto un cinese ignaro della lingua araba, esser logico che egli abhia subordinato gli ornati floreali, in senso verticale, ossia parallelo alla sua grafia nazionale. Ma più altento raffrouto eon le decorazioni della tomba principessa Bika $[$ † 1371], nella menzionata moschea di Varamin, e di Chal Sindelı in Samarcanda [1392], ecco risolvere di movo il dilemma in nostro favore; giacchè ivi non solo ritroviamo iscrizioni verticali contrastanti alla direzione degli ornati. hensi tipiche ripartiture, else, per la loro eontenenza. fammo quasi della stoffa scaligera e delle altre affini vere imitazioni tessili di codeste decorazioni parietali (14).

Inoltre, i draghi nella stoffa scaligera 

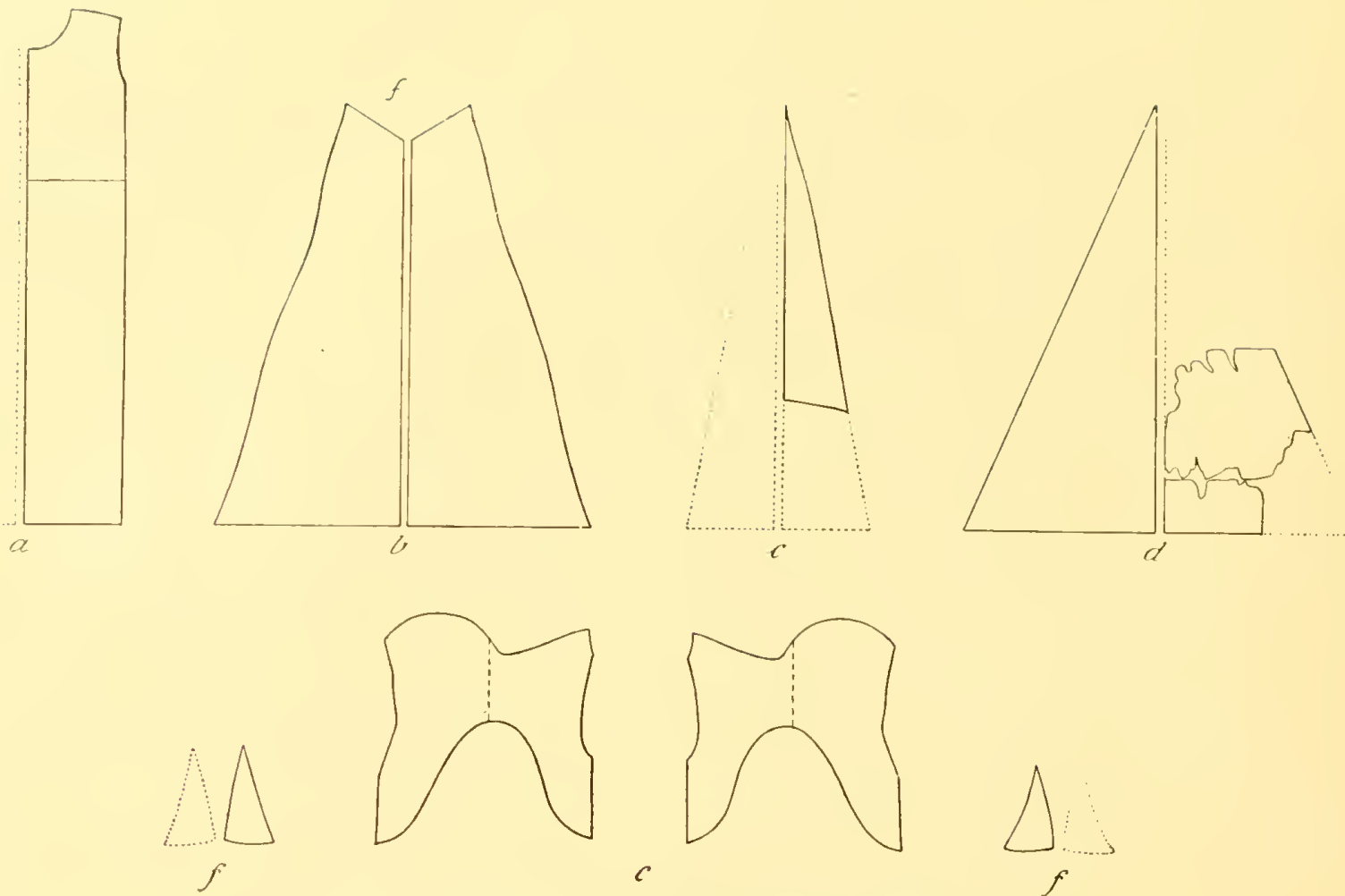

$c$

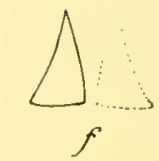

Fig. 12. - EI.EMENTI DELLA TLVICA MANICATA BLL E ORO

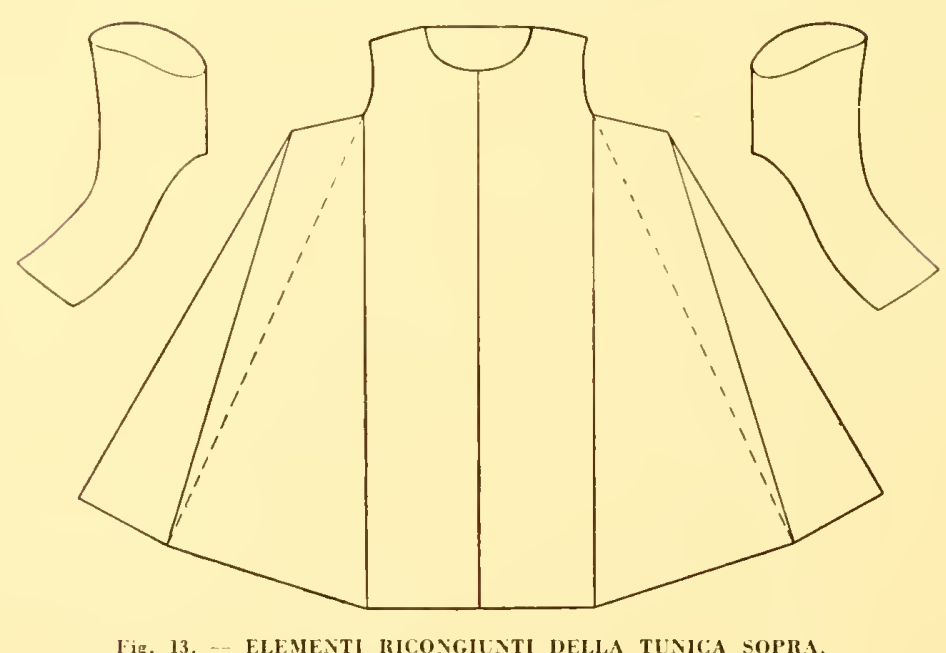

stanno per ismarrire i profili loro come già lontani dai modelli originali; mentre invece gli ornati arabeschi vi son tracciati con quella sieurezza propria a chi sa quel che vuolesprimere: e questi in notevole preponderanza sugli altri, onde l'indice del- la bilancia estetica volgesi vieppiù verso la sfera musulmana.

Ed ora, cosi edotti, chiediamo il sussidio di nuovi huni alle altre stoffe veronesi che per l'accertata comune origine tecnica sono autorizzate darci. 

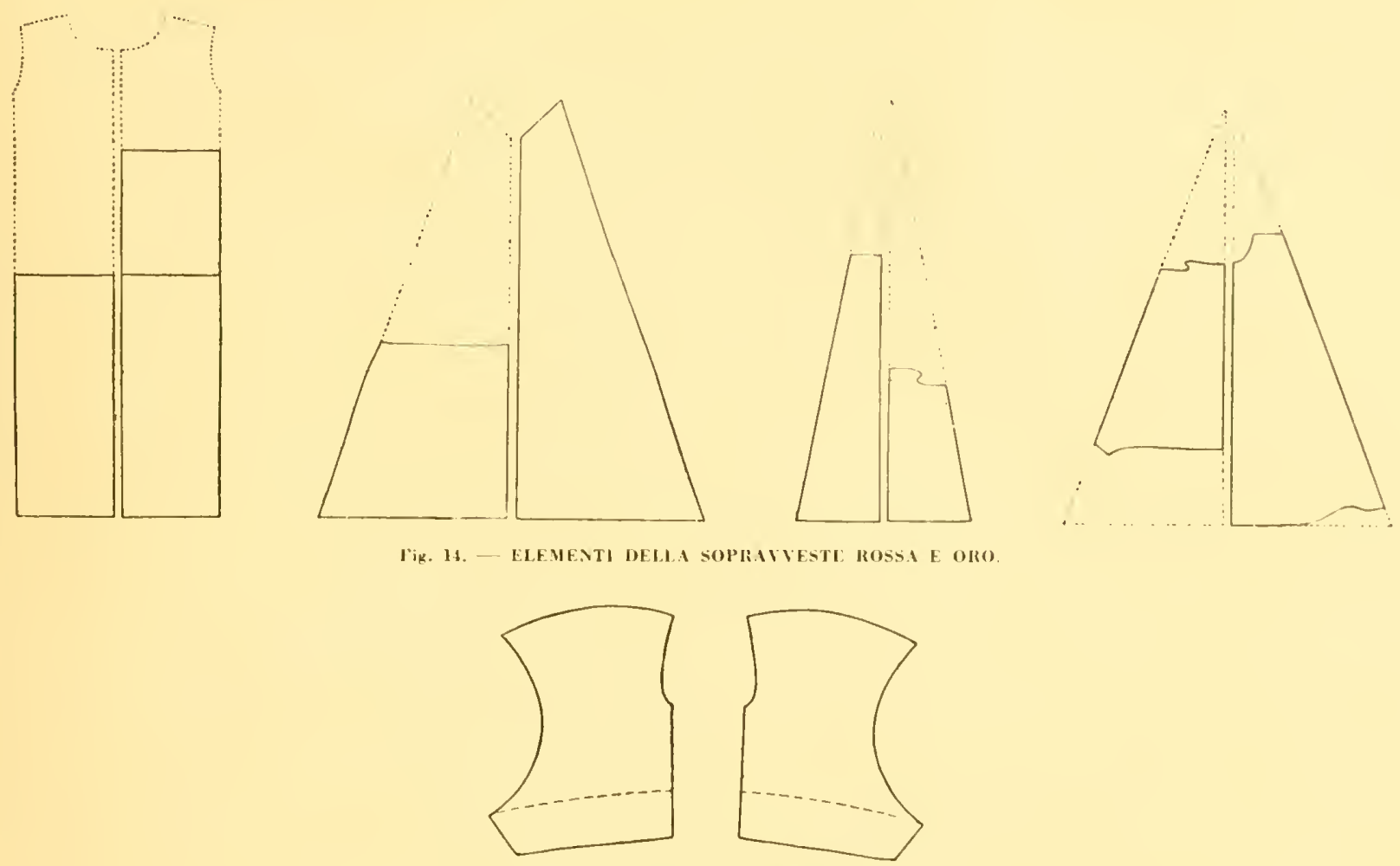

Fig. 15. - FLEMFNTI DFI, CAPPICCIO

Nel broccato D de argento et auro per usare una defunizione coeva - le formelle ammandorlate sono una delle corniei più tipiche dellarte iranica, che insieme alle iscrillevi palmette ritornano negli usufruiti termini di confronto. e singolarmente si rilevano germane di quelle negli scomparti maggiori del velo sealigero primo. A lor volta i noduli inestriealibi fra le formelle - derivati dal simbolo huddaico ichang - suggellano in un sol legame questo esemplare con gli altri di Ratishona, re ranti l’istesso marchio e gli stessi pesci: mentre i lioncelli miranti la mezzaluna ritornano nella stoffa 358 del Falke, ritemutavi persiana o mamelucra.

Il broccato E. sebben ridotto al solo ordito. adombra due tipi di pigne diversi derivati dalla simbolica sfera fiammegriante einese - quali si riseontrano pure in altri tessuli arabici. come in quallo di prove nienza tombale egizia seguato col nome di Mulanmed Nasir 1293-310 (15). A puesto proposito. secondo narra lo storico Mhulferla. emiro mameluceos. detto daliffo ricevette in rlono nel 1323 dal Gran Cante nongolo, per mezzo d"un ambaseria, ben selle cento pezze di stoffa; delle quali si puci individuare un saggio superstite nel drappo

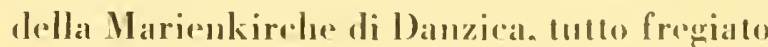
a draghi cinesi fra ucerelli addossati. resanti melle ali ma dedicatoria al prefato eatiffo Nasir, in caralteri cufici (16).

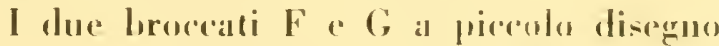
di animali, sidentificano nel tipo terenien ornamentale ad una stoffa rinvenuta nella Lomblia di Bencodetto Xl † 1304 in San Domenico di Perugia: la qual, stoffa ri- 
tiensi d'origine cinese. non contrastandovi la qualità papirifera dell oro intessuto (17). ehe nelle due scaligere - come si ¿̀ detto - ci risulta invece membranaceo.

Il broccato II ritorna spesso definito ad pineas rel folias negli inventarii sincroni come in quello della Chiesa di S. Francesco d"Assisi (18) -- fra panni tartarici che in senso lato riferivansi all"Oriente.

Il velo $\mathrm{B}$ e l’analoga stoffa ad listas de argento, escono per la specie del filo spirale dal gruppo definito. e sono da ascriversi, appunto per essa, probabilmente alla manifattura nostrale.

Volendo ora giungere ad una fondata conclusione. simpongono alcune ovvie eselusioni geografiche. Palermo. no: perchè ivi al tempo del gruppo scaligero. l"industria tessile languiva. e quella palatina. eui esso dovrebbe appartenere. era del tutto spenta; mentre son venuti meno a noi i riferimenti della vecchia teoria siciliana, dando luogo ad una tipologia del tutto diversa da questa in argomento (19). Lucca neppure: chè dopo fu presa nel 314 da Uguceione della Faggiuola. le sue maestranze sbandaronsi; e i tipi di codesto centro italico. se pur atlivo negli anni seguenti, ebbero di già as. sorbito nello stile araldico toscano i modelli sienlo-arabi. Venezia. infine, tanto meno, risorgendovi allora le prime tessorie in sulla tradizione lucchese, volgente a forme goticlue nuove (20).

Così l'industria tessile italiana appare, in eodesto periodo, in via di rinnovamento, mentre la sua teenica fissavasi con l'oro spirale filiforme, e con le eimose tipiche a cordelloni: caratteristiche altrettanto estranee al gruppo sealigero.
Nel quale gruppo. dandovi un ultimo sguardo generale. notiamo ancora una volta laspro contatto dellelemento islamico con l'estremo-orientale, che, in via logica, dol. hiano supporre avvenuto in una stazione internedia, ove le due correnti artistiehe, in direzioni opposte deflnenti, soffermaronsi esitando.

Tale stazione va dunque ricercata in Mesopotamia, o. più verosimilmente. in Persia. non solo per estrinseehe ragioni storiche, bensi perchè viciniore alla sfera d"influenza serica. Quivi appunto passando Marco Polo nel 270 già notava nel suo itinerario le industrie tessili in fiore, descrivendone la varietà delle opere e de" motivi (21); mentre contemporaneamente i testi arabi ne confermano il primato (22). E ancora. in linea indiretta. l"inscritta stof. fa di Ratishona legge in lingua più propria alla Persia ustad per maestro. mentre in altri centri susava meglio miallim.

Ma non pertanto, nè per le stabilite analogie fra il velo scaligero primo e larte iranica, si può del tutto escludere il vasto territorio della Siria e dell'Egitto sotto i Ma. melucchi, quantunque tali regioni fossero allora in crisi politica ed economiea, e le stoffe orientalizzanti ivi rinvenute in $\mathrm{El}$ Azan e in Dronka (23) si debbano piuttosto considerare importate che non ascrivere all'industria del luogo.

Limitianoci adunque a rimpatriare idealmente i bei broccati scaligeri. per mezzo - se volete - di una galea veneziana, deponendoli provvisoriamente presso le coste siriache, in attesa che la via carovaniera del ritorno sia più libera dalle attuali nebbie ipotetiche. 


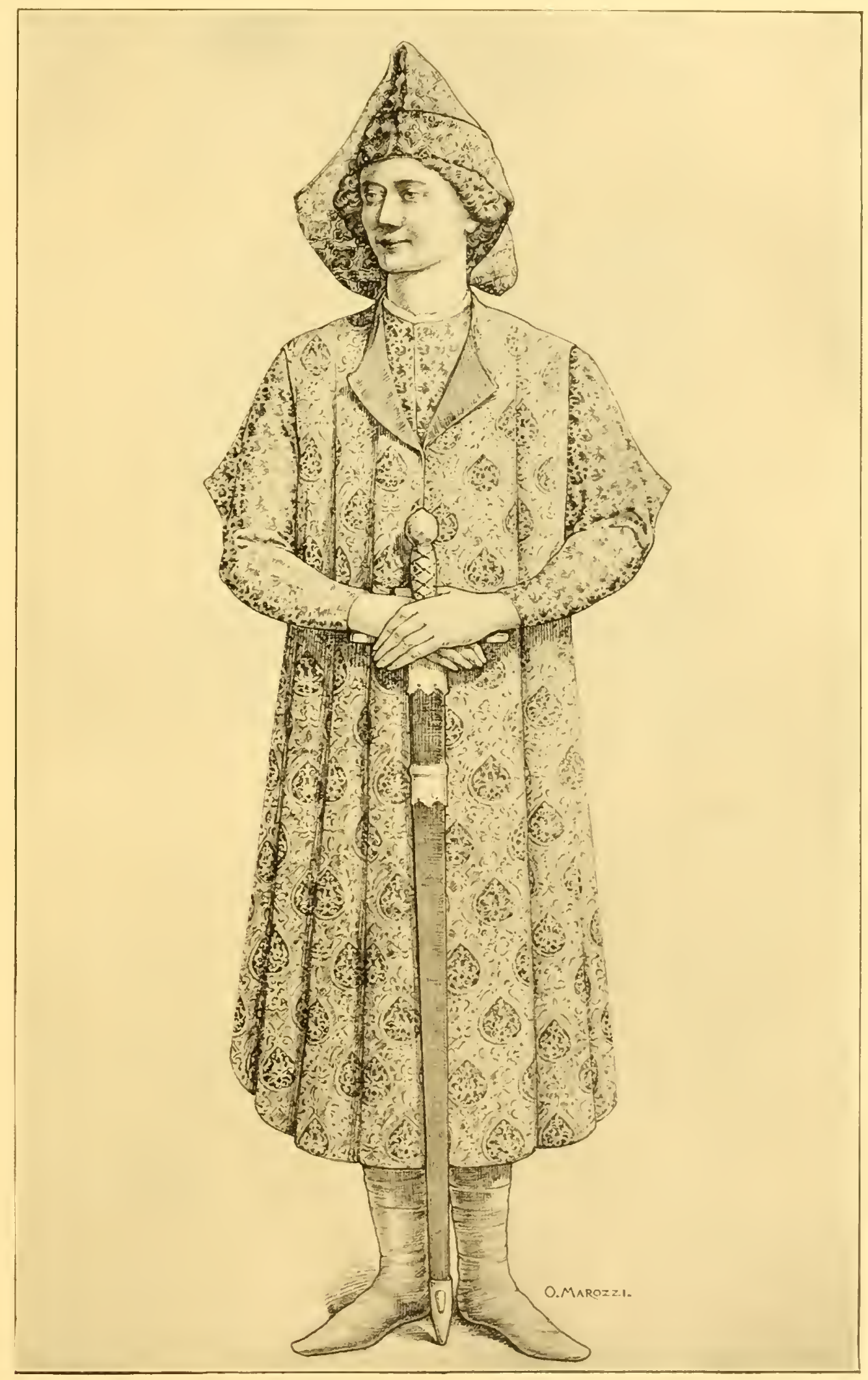

Fig. 16. - CANGRANDE « NOBILISEIMAVENTE D'LYA SUA ROBA VESTITO (Ricostruzione grafica dagli elementi originali). 
Studiati i diversi tipi di stoffe che formano il eorredo funelore di Cangrande. consideriamone $\mathrm{i}$ ritagli in rapporto alla foggia delle restimenta.

Notasi innanzi tutto che $\mathrm{i}$ varii elementi sono disgiunti e non serbano tracee di asole e di bottoni nè di punti. pur conservando i segni delle costure: aggiungasi poi che nimn d"essi fu ritrovato al suo posto d"origine. ma confusi e ammassati. in gran parte ai piedi della salma, eome se la tomba fosse stata manomessa.

Na nonostante il disordine. raggrupandoli secondo i motivi. abluam potuto rieavare primieramente dal broccato blu a piccoli disegni doro (F) il grafico fig. 12, integrato nelle parti maneanti dal presupposto che ogni singolo pezzo dovesse avere il suo gemello a riscontro: si ottennero cosi le sagome necessarie a mettere insieme una tunica manicata ampiamente eadente, o meglio gonnella. come la chiamavano ai tempi di Boceaccio (24).

La coppia di ritagli a ne stabiliscono intanto la parte anteriore indicata dalla inenrvatura dello scollo e dal garbo dell. Illa. niche; i due triangoli smussati $b$, o ghe. roni. conginnti ai lati del petto insieme ai minori $c$. anmentano la lalza della veste, compita dagli altri $d$. posti a tergo. I due ritagli e. ripicgati lungo la linea tratteggiata. formano le maniche. caratterizzate da una punta prominente alla parte posteriore del loraceio. e ehiusi ai polsi con lagginnta dei tasselli abbinati $f$. resa necessaria per il fatto che la larghezza del telo in cui furono ricavate non ne consen. tiva la capienza.

Cosi riordinati i membra disjecta, integrano lo schema geometrico fig. 13: il quale. salvo il tipo eivile delle maniche. corrisponde al modello talare della rife. rita tonacella di Ratishona e di quella liturgica di Sant'Elia. ove egualmente $\mathrm{i}$ gheroni dal verlice smussato. son cueiti alla parte anteriore ad ampliare il giro ascellare (25). Le misure che risultano dalla proposta rieostruzione sono: lunghezza della tmiea. m. 1.28: perimetro della gomnella. 111. 3.20: larghezza delle spalle, m. 0.18: le quali misure ben sattagliano ai comnotati else di Cangrande dà una cronaca contemporanea: "nomo di bell"aspetto e di alta statura e forte in guerra ". (26).

Oltre codesta prima tuniea si è potnta individuare una sopravreste nel drappo sanguigno a pigne d'oro $(\mathrm{H})$. simile alla precedente. ma priva di maniche. La figura 14 ne mostra gli elementi in relazione alle parti mancanti punteggiate. Se in origine avesse avute le sue larghe soprammaniche. aperte davanti. quali sono foggiate sul catalet. to di Cangrande. non siamo in grado dire se non quando verranno ricuperati alcuni avanzi delle vesti. che ci risultano lasciati nell'arca dopo lodierna ricognizione: nondimeno fin da ora se ne può anche anmettere lassenza. senza trasgredire alla moda treeentina qual'é documentata in dipinti coevi.

Passiamo ora allesame dei due pezzi gemelli ritagliati nello stesso broceato d'oro e argento del telo D. secondo le sagome figura 15. Eschuso il loro nesso colle suddette vesti. per la forma che in nessm verso consente combaciarvi. siam giunti a riferirli al cappnceio: si cuciseano infatti a mo" di sacco. col lato inferiore aperto, e si otterrà una specie di berrettone, ehe. revoluto acconciamente intorno al capo, lun- 


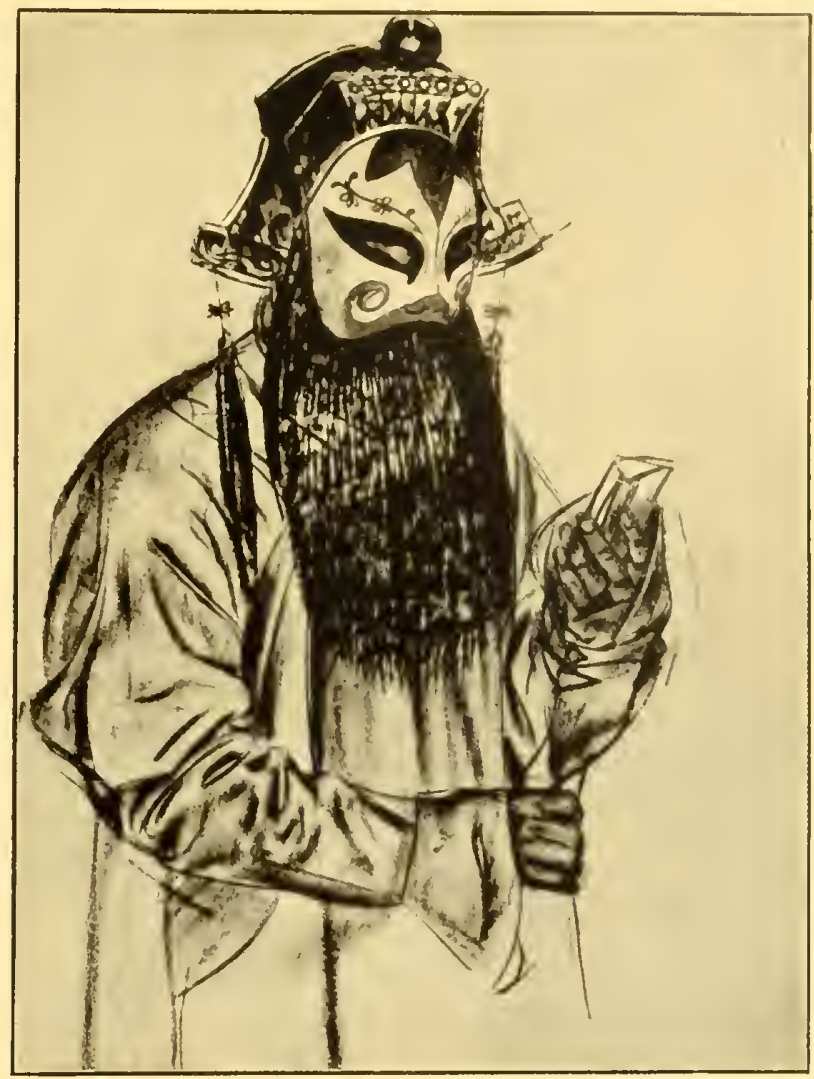

Fig. 17. Costune di UN GUERriero CINESE, CHF, RICORII 1I. THO DELLA MAVCA DI CAVGRANDE.

go la tratteggiata. forma il mazzocchio; mentre la maggior ricchezza che ne avanza, cadendo rasente la gota sinistra. secondo liuso, va a lambire la spalla (27).

Il raffronto con raffigurazioni pittoriche e segnatanente con gli affreschi di Ambrogio Lorenzetti nel Palazo Commuale di Siena [1337-39]. non contraddice alla nostra restituzione delle vesti e del cappuccio relativo (fig. 16); mentre, dati i profili combacianti delle varie parti, non ei sembra possibile proporne altrimenti sintassi più semplice.

Solo la divisata foggia delle maniche puntute ai cubiti si distacca dal comune, e saccorda al gusto orientale delle stoffe identificandosi al taglio di certe giacche cinesi usa- te da guerrieri (fig. 17) (28).

Codeste esotiche riecreatezze faumo pensare a una speciale predilezione per l'arte cinese stimolata forse dal recente ricordo delle meraviglie della corte del Gran Canc mongolo, narrate con imaginosa fedeltà dia Marco Polo nel suo Milione; a sembrano quasi adombrare l'origine dello strano nome dello Scaligero impostogli forse per antonomasia.

Estranei al contume cosi carallerizzato. rimangono due elenenti di cui non appare evidente l'originale destinazione: a sono il telo blu-cupo a pigne (E). e il croeero a mi. nuti disegui (G). cui fan seguito due tasselli dell’istessa stoff i.

Come semplice ipotesi, si può pensare che 
il primo fosse inserito nella tunica manicata al posto del dorso. giacchè la larghezza $(0.48)$ collina con quella del petto: e per altro il suo cattivo stato di conserva. zione è tale da far supporre abbia subito il decubito della salma. Del resto. vesti di diversa specie tessuti si riscontrano e nelle miniature e nei dipinti stessi del Loren. zetti; e nel caso presente è più ammissibile la discordanza. per il fatto che la tunica blu veniva coperta a tergo dalla sopraveste rossa. D’altra parte è lecito anche l'inferire - data la rarità di codeste stoffe oltremarine - che il broccato non fosse bastato alla confezione dellintero indumento; tanto più che la sopravveste risulta dal disegno rovescio di un gherone, tagliata in economia: e ciò appunto per la sezione trasversale al telo producentevi in poca lunghezza due triangoli eguali in senso opposto.

In quanto all"altro telo croceo. non trova nessuna logica ubicazione nei descritti indu. menti. nè si può attribuire con certezza al letto funebre compiuto dalle stoffe A, B, C e D.

A questo proposito s'impone il ricupero degli avanzi. che, come abhiam detto, furono lasciati nellarca: coi quali forse sarà facile risolvere il problema di questi due pezzi sbandati. o comunque completare le parti mancanti delle tuniche. salvo in quei tratti distrutti dal tempo.

$\mathrm{U}^{\top}$ n particolare di non licve importanza

11) L'atto di ricognizione è pubblicato da A. AVENA. La salma e la tomba di Cangrande I della Scala, in: Dante e Verona, Verona 1921.

(2) Per le tessili iscrizioni arabicbe in genere. ffr. GUEST, Notice of some arabic inscriptions on textiles at the South Kensington Museum, in: Journal of the R. Asiatic Society, 1906; pag. 337. occorre rilevare dallesame dei frammenti cosi ordinati c ricongiunti: cioè che non rimane fra d'essi alcuna vestigia di fodere; le quali non è attendibile siano andate totalmente marcite, quando han resistito nelle istesse condizioni tessuti ben più effimeri e leggeri. Questa assenza in rapporto al fatto già notato. che ogni parte del vestiario è scucita e priva di asole e bottoni. e che le vesti sono alquanto risigate. noi la spiegheremmo ricostruendo nel seguente modo i pietosi uffici che precedettero la tumula. zione di Cangrande.

Il 22 luglio del 1329 egli morì in Tre viso: indi la salma. trasportata a Santa Maria della Pecana. venne imbalsamata - «aromatis arte refectum ) (29) — e avvolta nelle bende di due specie di lini, quali forse eran presti in quel luogo. Il 25 seguente $\mathrm{fu}$ tumulata in Verona (30); e poco innanzi, ritagliate espressamente le vesti sepolcrali, messe insieme in fretta con qualche imbasto e senza fodere. le adattarono alla meglio alle spoglie ormai irrigidite (31).

Poscia, con mano tremante per la triste bisogna. dal broccato oro e argento, praticatovi un taglio. interrotto da un impeziente sgarro (32). ne spiccarono un telo, che, steso al fondo del sarcofago cosparso d'erbe aromatiche, accolse la salma; e questa cover. tarono d'un altro prezioso drappo inaugurato da una pezza intonsa (33). sul quale, infine, il tempo distese il suo incorruttibile velo nero.

(3) Secondo il prof. M. Guidi. l'ipolesi di un imita zione fantastica di lettere arabiche a seopo ornamentale par certo sia da escludere, mentre egli propenderebbe a ritenere l'iscrizione una delle solite ele rieorrono in oggetti artistici. auguranti gloria, felicita, lunga vita, ece. a colui il quale quelli eran destinati, ma corrotta e resa illeggibile causa la poca fedeltà eui un eventuale ori- 
ginale può esser stato riprodotto da un artefice impe rito di serittura iraba.

Ma il dott. E. Kuhnel di Berlino, considerando il valore calligrafico dei caratteri, non anmette la corruzione del testo come dovuta a imperizia, e suppone che l'iserizione, forse sacra, sia stata appositamente storpiata per. ehè i cristiani, ai quali la stoffia, qual prodotto di esportazione, era forse destinata, non avessero a profanarla.

Il prof. U. Monneret, invere, invertito l'ordine di al"me parole leggihili, ne rirava un trisagio riblotto, attri. buendolo, qual eriptogramma, a un arabo-eristiamo ehe per prudenza abbia voluto dissimulare l'abiura.

Fra tali dubbiezze, noi ci asterremo dall'appoggiare la tesi dellorigine di codesta stoffa su dati filologiei, auche per non incorrere in nuove deviazioni di giudizio, dopo l'esperienza della falsa lezione Karabacek - else riferiremo nelle pagine seguenti - responsabile della leggemdi siciliana relativa ai tessuti di Ratishona. Nondimeno. seb. bene ignari di lingua araba, crediano essere più prossimi al vero, spiegando nel modo seguente l"incongruenza fra il earattere litterale e il contenuto storpiato delliserizione.

Innanzi tutto oceorre riportarei all'offieina da eui usei il lessuto in questione, e seguirne verosimilmente le fasi preliminari alla sua lavorazione; le quali s'iniziano con l'opera del diseguatore, intento a traceiare con meditata cura le parole dettategli, elie egli ripete in zone parallele longitudinali al telo, secondo l'uso orientale, colmandlo gli spazi fra l'una e l'altra con gli usufruiti ormati che già possiede. Poscia il disegno, cosi composito, passa al tessiere chè lo metta in opera: ma la prima obiezione che questi dovrà fare nel considerarlo, si è che la leg. genda supera in lunghezza il passo massimo del suo telaio. adeguato alla seguenza di notivi normali. Allora - come inducono a eredere altri tessuti dagli ornati contratti, sem. plifieati e talvolta deformati - egli non esitera a mutilare la leggenda riducemdola con garlo estetico al necessario rapporto; ma senza tener conto delle ipotesi complicate cui il suo ingenuo lavoro di cesura potrà a distanza di secoli suseitare.

(4) R. ROGADEO, Il tesoro della R. Chiesa di S. Ni. cola di Bari, in: L'Arse, amo V, fase. IXXX e XI.XlI.

(5) STRZYGoWSKI, Seidenstoffe arts Aegypten im Kaiser Friedrich Museum, in: Jahrbuch der Preus. Kunsts., 1903.

(6) Da una cronaea edita dal BARTOLOTTI e riferita da GANDINI, Ars textrina, Roma, 1887.

(7) DREGER, Europäische W"eberei und Sticherei, Wien 1904, tav. 187.

(8) MUNSTERBERGER, Chinesiche Kunstgeschichte, Esslingen, 1910, vol. II, pag. 339.

(9) FALKE, Kunstgesehichte del Seidenweberei, Berlin, 1913, vol. 11, p. 50 e segg.

(10) Vedi il mio articolo precedente, Oro testile, ece. Anche il prof. Vacea sinologo della R. Università di Roma, ebbe a confermarmi ehe: nel XlY see. in Cina non si è mai usato l'oro membranaceo. Per altro, quello pa- pirifero cinese, per la sua struttura laminare lo troviamo definito oro batuto ne I liaggi di M. POL.O.

(II) BUSHELL, L'are chinois, l'aris I9]0, pag. 22.

(12) MARTIN, A history of oriental carpets, Vienus 1908, eap. Il (The art during the dominion of the Mon. gols orer l'ersia).

(13) SARRE, Denkmäler persichen Baukunst, Berlin, 1901 .

(14) SALADIN, Manuel d'are Musulman, vol. I, eap. IV.

115) FALKE, op. rit., vol. Il, fig. 360.

(16) KARABACEK, Die liturg. Gencünder mit arab. Inschriften in Danzig, in: Mitteilungen des Oesterr., mus., V.

117 Chinese and Japanese brocades, in: Bulletin of the Metropolitan Museum, vol. XV, n. 2. Cfr. pure per il tipo della tes-itura e del disegno FALKE, op. cit., vol. II, fig. 321 .

(18) FRATTINI, Storia della Basilica e del Comento di San Francesco d'Assisi, l'rato, 1882.

(19) Animali addossati e allrontati entro dischi, di earattere bizantino-arahico le caratterizza: efr. FALKE, op. rit., vol. 1, p. 119 e segg.

(20) BINI, I Lucchesi a V'enezin, Lucea, 1853.

(21) M. POLO, I ritggi: "Quivi lin Yezd) si la. vora drappi d'oro e di sita clue si portano per molte contrade "; "Le donne [di Caramania] lavorano tutte cose a seta e ad oro e a urcelli e a bestie, nobilmente; e lavorano di cortine e di altre cose molte riceamente; e coltri e guanciali e tutte cose ".

(22) Debbo questa notizia al prof. Monneret, e la se. guente al dott. Kuhnel.

123) FALKE, op. cit., vol. 11, pag. 54.

1241 MERKEL. Come restirano gli nomini del "Decameron ", Roma, 1898: "La gomnella era usata tanto dagli nomini ehe dalle domne; quella da nomo era piuttosto lunga ed aveva maniche ".

(25) BRAUN, Die liurgische Gemandung, 1907, 75.

(26) ORTI-MANARA, Cronaca inedita dei tempi degli Scaligeri, Verona, 1852, pag. 11.

(27) VARCHI, Storia fiorentina, ed. Milanesi.

1231 CIll.CHIA.CIIIEN, The Chinese thertre, Londo11. 1922.

(29) IERRETO DE' FERRETI, le Opere: Istimto Sto. rico Ituliuno, pag. 100, V. 352 trifer. dall'AVEXA nell'op. rit.).

(30) La salna par certo sia stata messa prowisoriamente nella tomba dei padri in Santa Maria Antica, indi a pochi anni traslata nell'attuale area monumentale sulla porta della stessal rluiesa lefr. AVENA, op. eit.l.

(31) L'uso d’imbastire in fretta le vesti, se non al defunto, a rhi doveva accompragnarlo in gramaglie alla sepoltura, dava a guesti il nome d'Lmbastiti.

1321 Codestil azione drammatira ei viene suggerita dalle riferite earatterintiche del hrocento stesso lettera D.

(33) Diro intonsal perchè reea, rome si is viso piin : .1. pra, la testata del telo col marebio del mercante. 

VELLUTI AULICI 



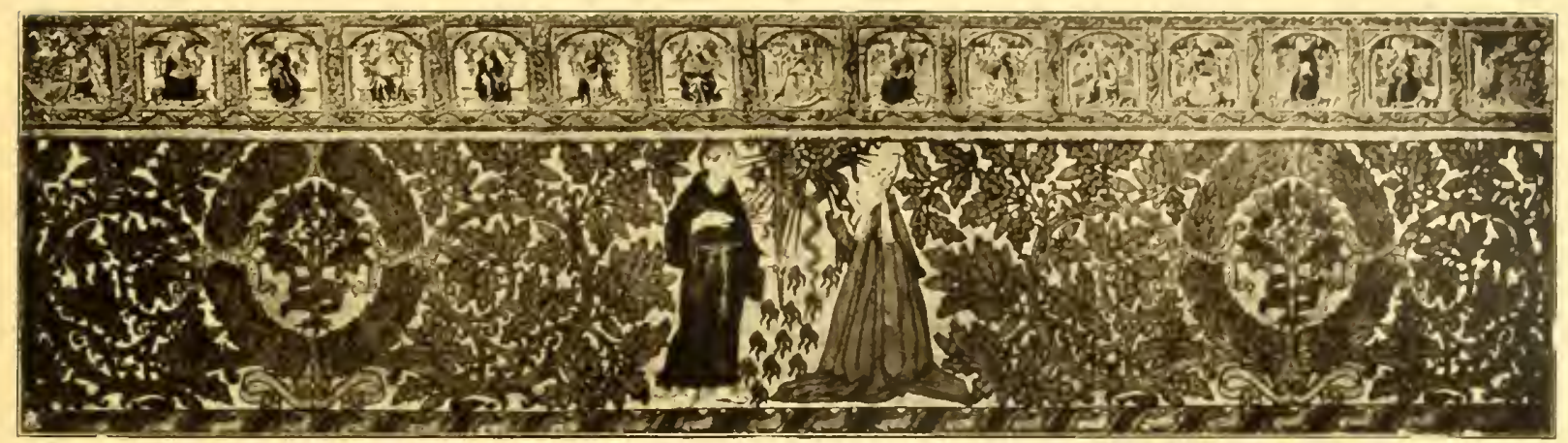

Fig. 1. - PAllotto DI SIsto IV (Basilica di Assisi).

\section{VELLUTI AULICI (*)}

I precipui caratteri generici dell'arte tessile nei secoli passati, si distinguono per una propria flora ornamentale, dovuta non solo a necessità tecniche e a un senso di riservatezza schiva di rapporti con le industrie affini, ma ben anche al libero affermarsi del gusto del colore, astraente dalle forme precise ed oggettive. Lento ne fu lo sviluppo per opera di mmili ed oscuri tes. sieri; i quali, se non legarono il nome ai singoli prodotti, diedero all arte loro fisionomia nazionale per eccellenza, tipica e varia di colori quanto italiche primavere.

Ma oltre le variopinte messi, cresciute al. laria libera, si vollero coltivare in chiusi tepidarii rare specie per diletto di pochi magnifici signori: ciò sono le stoffe di stile illustre, dense di quella special ricchezza pomposa delle cose regali; le quali, sebben elette, riflettono piuttosto lintenzione adulativa degli artefici e la vanità dei committenti, che non meglio il tipo genuino dellarte spontanea e corrente. Primeggiano fra queste speciose opere, che intitoliamo auli-

(*) Dalla Rassegna d'Arte, anno 1920, fasc. 9.10. ehe alle corti che le accolsero e le favorirono, i drappi aurei qui rimiti in esame, e che ai loro tempi si definirono enfatica. mente ( $i$ sovrani velluti del mondo ).

All'arte di Antonio del Pollajuolo deve la precedenza il paliotto regalato nel 1475 da Sisto IV della Rovere alla basilica d'Assisi (fig. 1). Nel mezzo vi è affigurato il pontefice genuflesso in adorazione di S. Francesco: ai lati, due corone di quercia incorniciano l'araldica rovere, avvolta il fusto d'un filatterio inscritto SIXTUS IIII PONT. MAXIMUS: sottostanti ad esse corone. ceppi hipartiti dilatano frondenti rami carichi di ghiande, rattenuti da nastri svolazzanti. Qual frontespizio. sovrasta il palio e l’illustra uı fregio in ricamo, diviso in qualtordici scomparti: con la Vergine e il Bambino, il me. diano; con Santi. i laterali: con le armi papali sorrette da angioli. i due estremi. Pure in ricamo su seta riportata. sono i volti e le estremità delle due figure titolari, non arrivando la tessitura a finezze fisionomiche.

La qualità del drappo ì Ia più ricca che telaio possa dare: fondo aureo, ornati cann- 


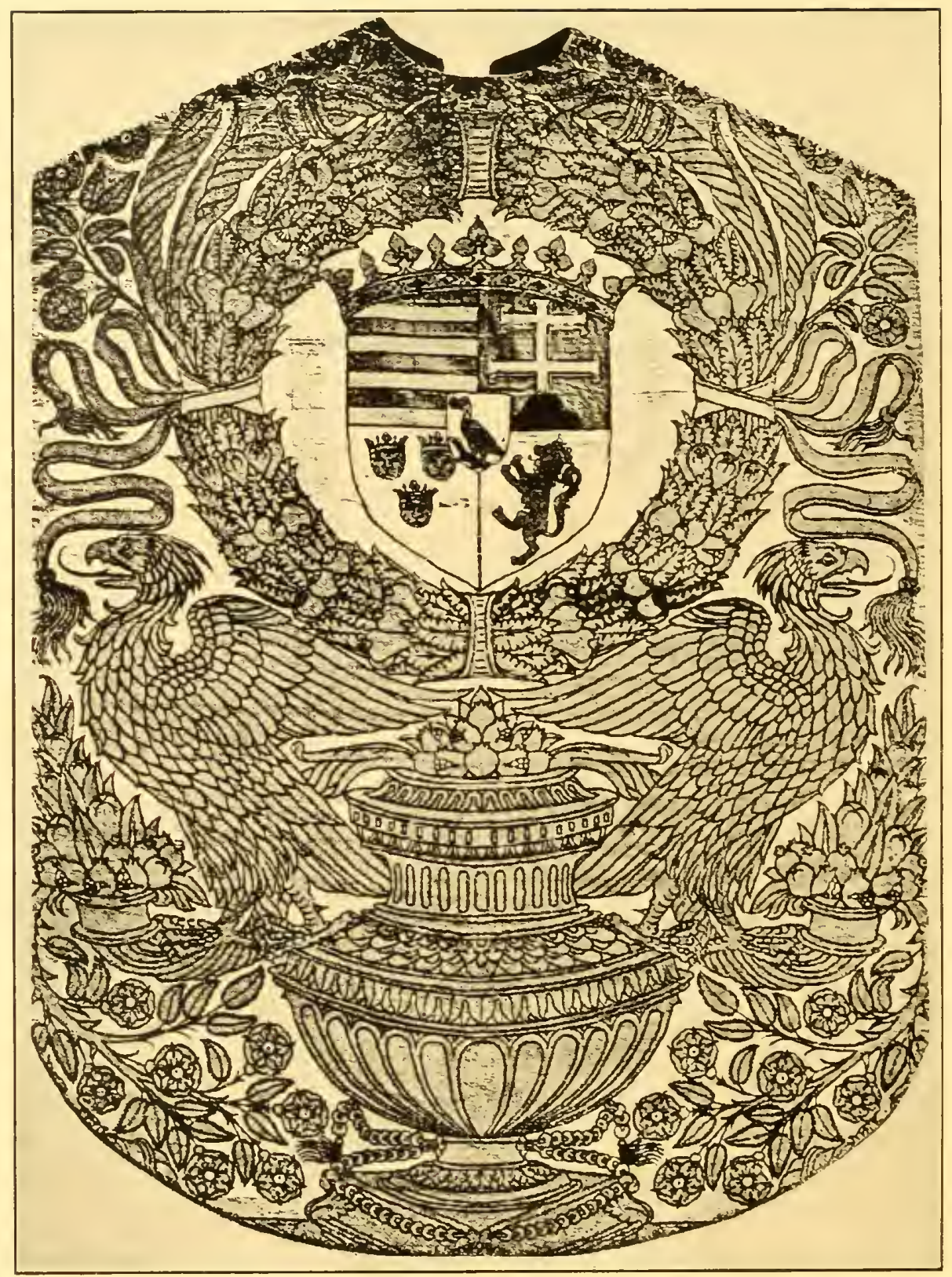

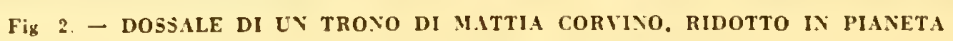
(Castello reale di Oefen, presso Budapest).

piti di foltissimi riccetti d'oro e d'argento. e delineati in velluto rosso.

Il disegno nitido e preciso nelle parti ri canate, quasi inciso col bulino; la stretta somiglianza stilistica della figura di S. Francesco con quella di S. Jacopo nella tavola del Pollajuolo, fatta per la chiesa di San Miniato; e più particolarmente le estremità del Santo ossute e nervose con il mignolo della destra contratto. e la testa del mede. simo dagli zigoni sporgenti - seguendo il Venturi - sono caratteri sufficienti per non 


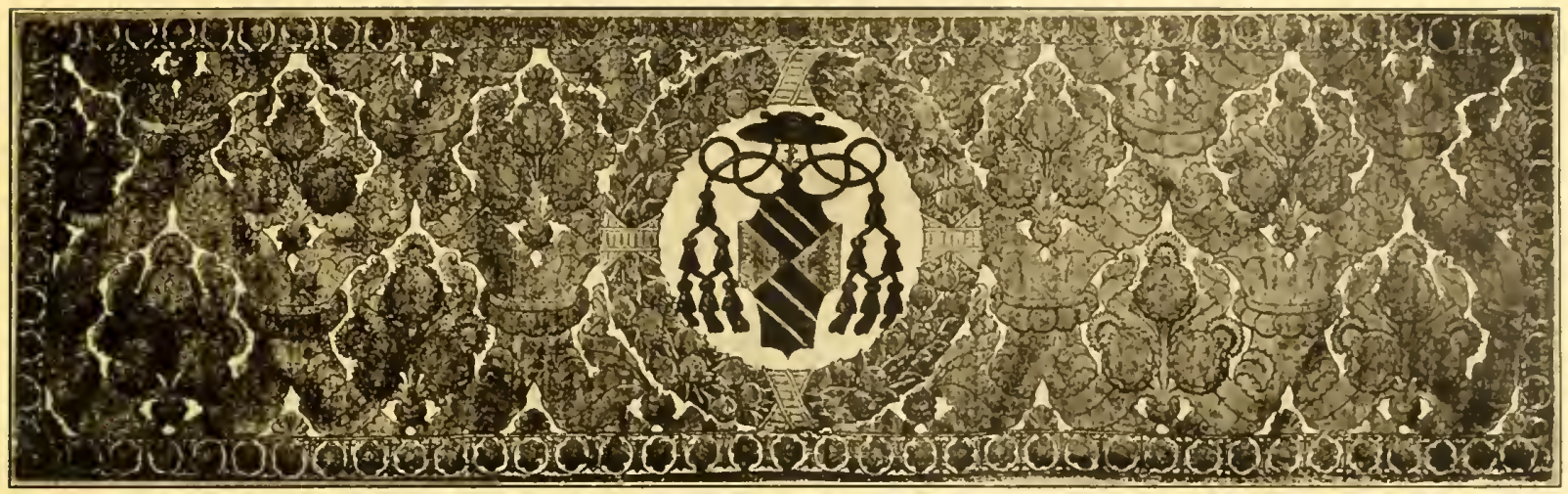

Fig. 3. - PALjotto DEllarcivescovo PEDRo govzales DE MENDOZA (Duomo di Toledo).

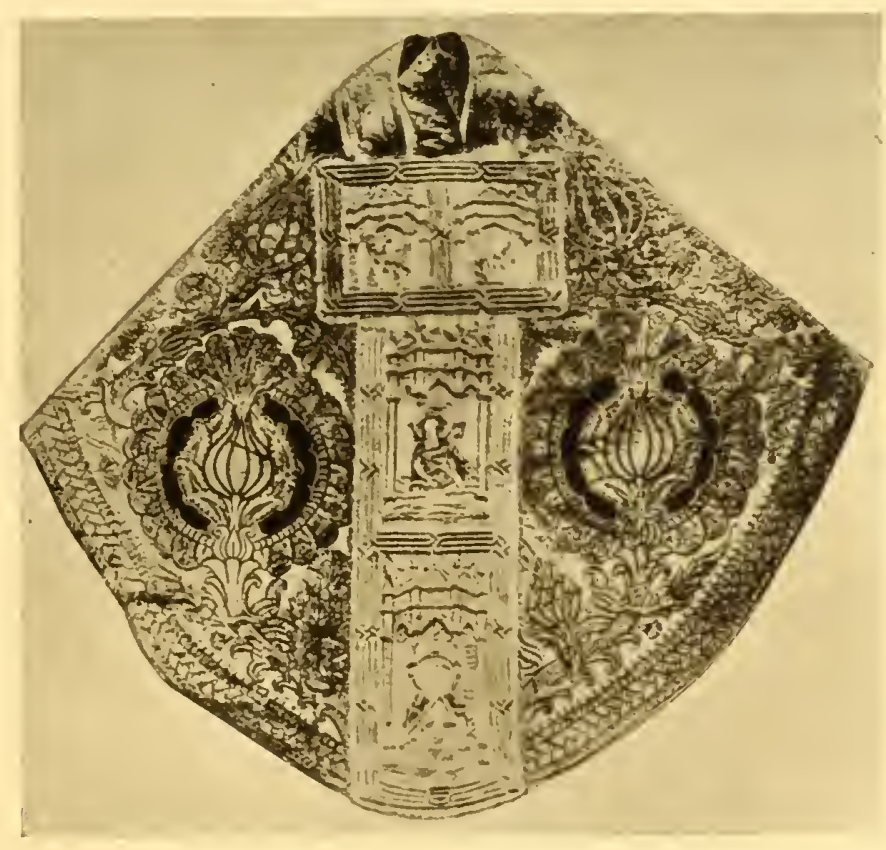

Fig. 4. - CASUla Di CAlisto III (Velencia).

ammettere dubhi sulla paternità del earto. ne originale di codesto palio (1). Anche molteplici appaiono i punti di contatto fra le figure ricamate nel fregio relativo, con quelle delle Virtù disegnate in parte da Antonio per ornare la Sala del Magistrato della Mercanzia.

Allattribuzione del Venturi acconsente pure il Falke (2), e la estende per ben fondate analogie a due gemelli dossali di trono, fatti espressamente per Mattia Corvino, re d"Lugheria 1458-1490: liuno, integro, in possesso della faniglia Erdödy (3): laltro, ridotto in forma di pianeta. nella cappella del Castello di Oefen, presso Badapest (fig. 2): entrambi nella tecnica elaborata identici. e simili nello stile grafieo al paliotto d'Assisi. 


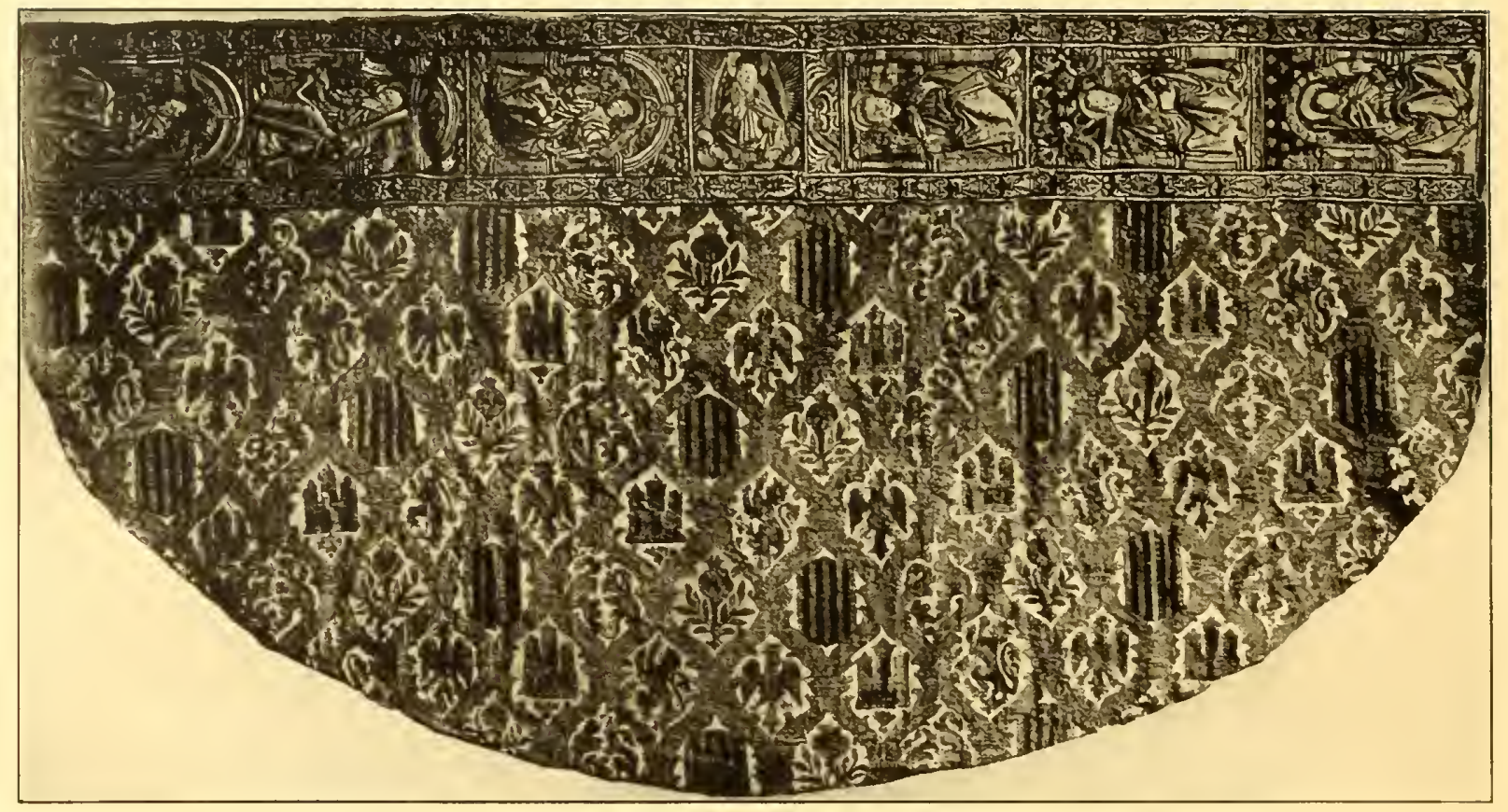

Fig. 5. - PIVIALE doxato DA isabella la Cattolica al prino vfocovo di gra.iata

(Museo storico dei tessuti, in Lione).

Mostra il primo esemplare Erdödy lo stemma reale entro corona di quercia e frut. ti; sotto cui campeggia un vaso a baccelli e a squane, fiancheggiato da superbe aquile che poggiano sopra cornucopie colme di melagrane, fra rami di rose e nastri svolazzanti: il tutto, incorniciato in una nicchia sorretta da pilastrini aventi per base un pavimento di formelle quadre in prospettiva fuggente. Il ricco disegno intricato, ma nitido, proprio all'incisione e al niello, e lo scorcio del pavimento, tipico nelle tarsie corali, come in quelle di Monteoliveto, fan quasi dimenticare la natura tessile dell'opera. ritraente dalla fornı, più clıe dal colore, la maggior ricerca di effetto; la quale ben si addice, del resto, all’immobilità architettonica dei seggi e degli altari cui cotesti drappi eran destinati nelle grandi solennità, mentre invece la varietà del colore più pittoricamente vuol vivere solo nelle mobili vesti dei personaggi.

Tornando alla attribuzione comune ai tre drappi, la corrobora un passo del Vasari nella vita del Pollajuolo, ove dice che: “ col disegno di costui furono fatte per S. Giovanni di Fiorenza due tonacelle ed una pianeta e piviale di broccato riccio sopra riccio, tessuti tutti di un pezzo, senza alcuna cucitura; e per fregi ed ornamenti di quelle furono ricamate le storie della vita di San Giovanni ). Ed infatti codesti fregi, esistenti tuttavia nell'Opera di Santa Maria del Fiore, offrono al confronto perfetta identità di stile con i ricani del paliotto d'Assisi; il cui broccato riccio sopra riccio è appunto inconsutile, e non in teli cuciti secondo luso consueto.

Altresì in un sol pezzo di broccato riccio figurato troviamo descritto un piviale nel li- 


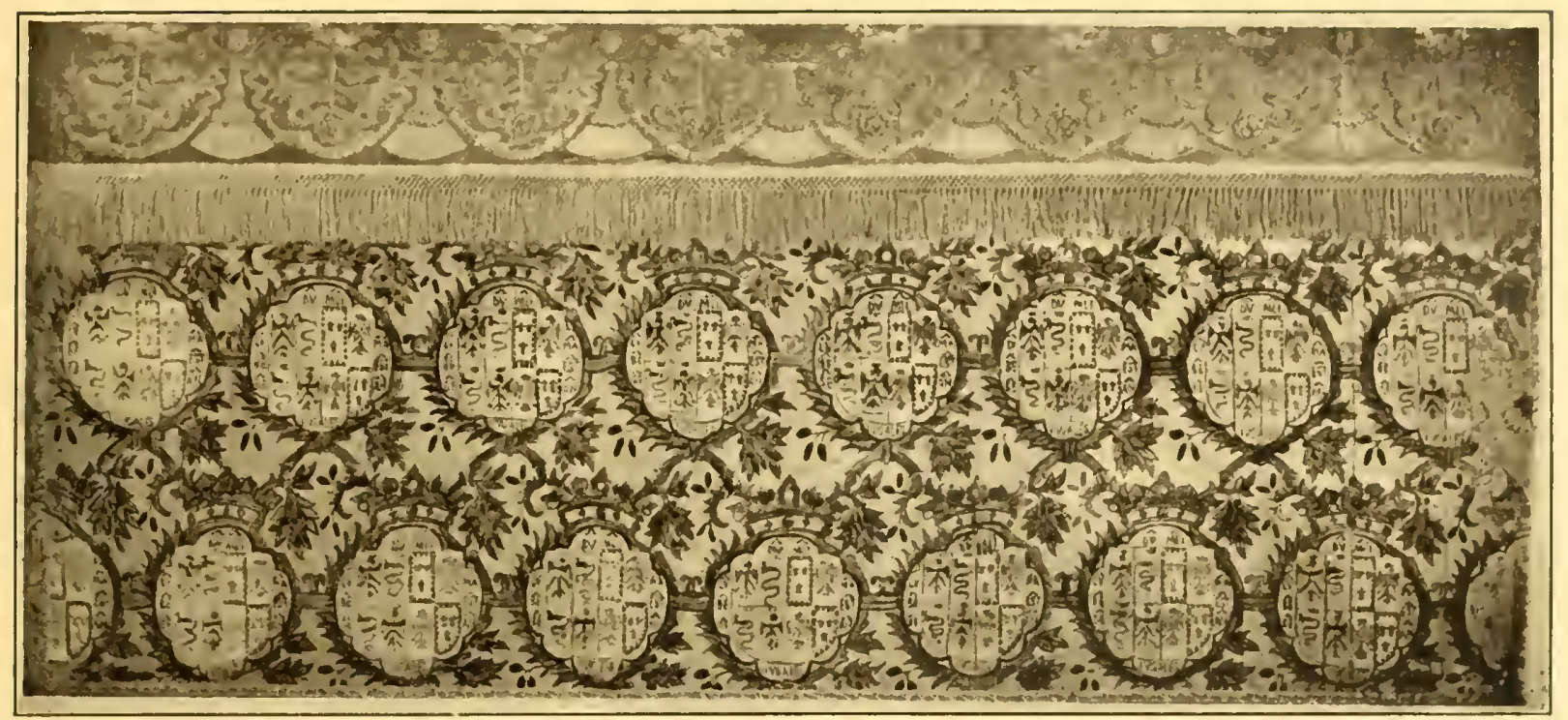

Fig. 6. - PALlotTo DI LLDOVICo IL, NORO F. REATRICE D'ESTE

(Santuario del Sacro Monte, sopra Varese).

bro degli introiti e delle uscite sotto il pontificato d'Innocenzo VIII [1484-92], successore al prefato Sisto IV: ( pro uno piviale in un petio brochati auri et argenti ricci eum Baptismo »... (4). Ed ivi apprendia. mo anche come lamore del fasto. spingesse detio papa a pagare per undici canne de brochato auri ricio ben altrettante migliaia di ducati doro (5). Fra le meraviglie tessili che gli appartennero, a precisarne l'im. pronta personale, va ricordata una cintura di spada, intessuti d"amlo i lati in rosso oro e argento, il suo stemma. il nome e l'anno 1491 in cui fu donata al langravio Guglielno I d'Assia (6).

Come sintende. le stoffe più ricereate e di maggior valore intrinseco erano allora i broceati riccio sopra riccio che usavansi talvolta in pagamento invece del numerario (7). Fra queste, altri saggi cospicui si ammirano nella casula di Calisto III [1455-58] in Valencia, appositamente tes. suta col suo passamano svolgentesi in curva alla balza inferiore (fig. 4); e nel paliotto del dnomo di Toledo, dedicato all'arcivescovo Pedro Gonzales de Mendoza † 14951. detto il Re di Castiglia (fig. 3). Ivi il suo stemma campeggia - girato dalla solita corona policarpa - in campo estofado d'oro a tronchi conserti carichi di foglie lobate e cardi fioriti; e lo stile ornamentale nella sua robusta grandiosità s'adegua al denso velluto di ben tre spessori soprarriccio qual bassorilievo tessile.

Sempre alla stessa tecuica e alla manifattura nostra, appartiene il piviale offerto da Isalella la Cattolica al primo vescovo di Granata. in occasione dellesodo definitivo dei Mori nel 1492: il quale piviale (fig. 5) - nel Museo di Lione - mostra. fra rami piegati a losanghe attraverso coronc, lo stem. ma della regina con quelli delle proviucie liberate di Leone, Castiglia. Aragona e Gra. nata.

Un altro capolavoro di oreficeria tessile. cosi finemente cesellato da sembrar nscito 


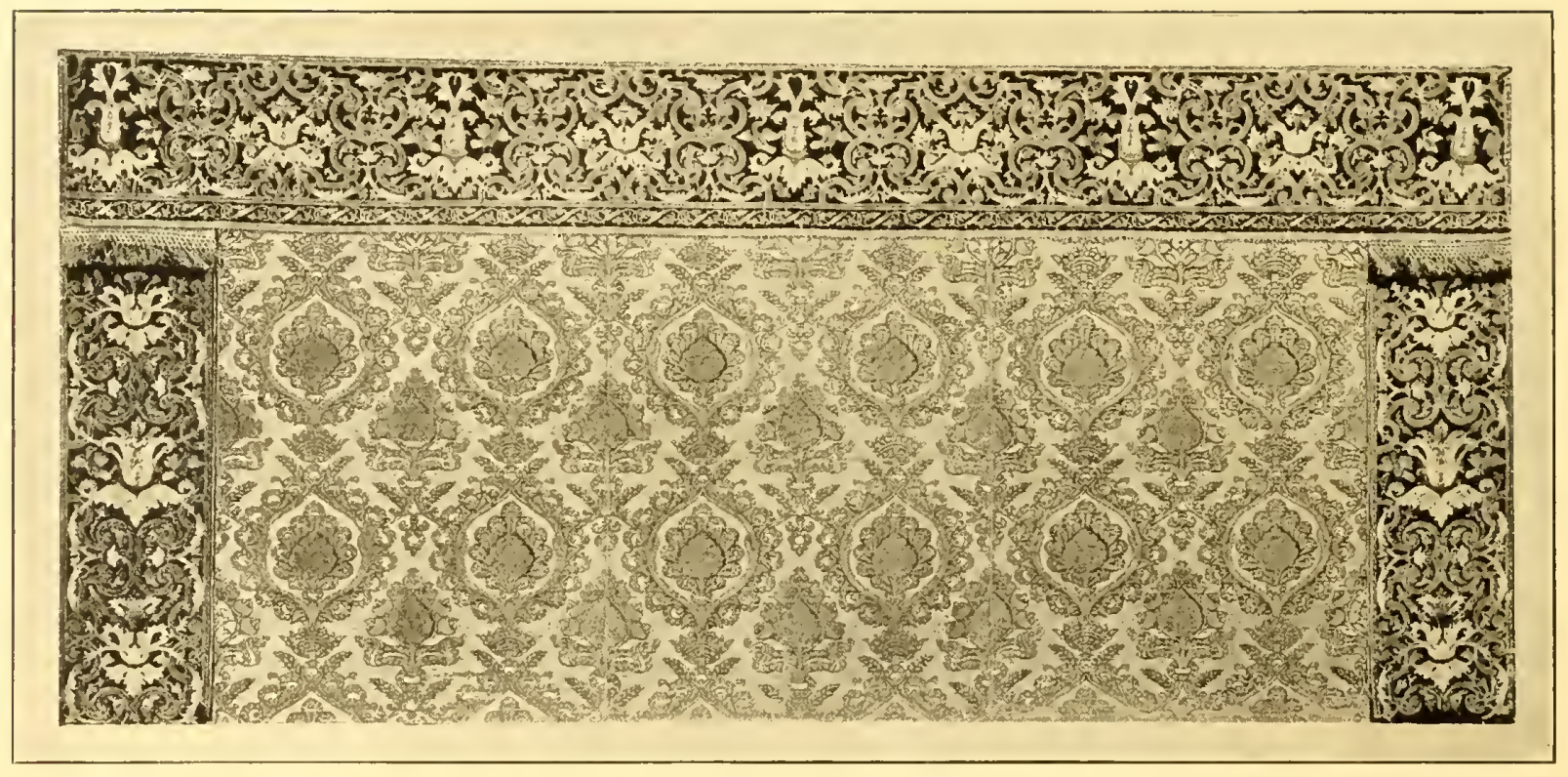

Fig. 7. - PALIOTTO SForzesco (Collezione privata).

dalla medesima fabbrica del menzionato palio di Sisto IV, o dagli stessi armadii del suo successore, tanta n`è la ricchezza, si riconosce nel paliotto donato da Ludovico il Moro e Beatrice d'Este al Santuario del Sacro Monte, sopra Varese (fig. 6). In esso, fronde di lauro incorniciano le armi abbinate delle due famiglie gentilizie, e recano intorno i monogranmi DV MLI LV BE SF MA EST AN JUGALES. che si sciolgono: "Duces Mediolani Ludovicus Maria Sfortia Beatrix Anua Estensis Jugales ». Il fondo del broccato è d'oro, come pure gli ornamenti in soprariccio, profilati di velluto rosso; mentre gli stemmi, contesti in seta policroma, sembrano di smalto. In ricamo n'è invece la testata con l'impresa della spazzola e del caduceo, fra biscioni aff rontati.

Allo stesso telaio, onde usci cotesto broccato, si deve certamente quest'altro, qui riferito a fig. 7 , risultando di teli della mede- sima opera e precisa altezza del primo ( $\mathrm{cm} .57)$, cuciti in tre pezzi che formano il solito paliotto; ma il disegno ne ritorna in più modesta edizione, priva degli stemmi e con le sole corone ducali alla sommità di ghirlande di fiori e spiche, entrovi pigne alzate d'argento con penne intorno. Questo il classico motivo milanese, quale possiamo riconoscere in dipinti. e che varia dal veneziano - meno determinato e più coloristico - per la maggior precisione grafica nel raggruppare e distinguere gli elementi floreali. Larghi fregi di velluto chermisi ricamato doro a disegno mistilineo con uccelli e festoncini, limitano tre lati al frontale; e, sebbene posteriori al broccato di circa mezzo secolo, sono pur essi di gusto lombardesco.

La tecnica di questi due tessuti sforzeschi par rispondere precisamente a quella di un famoso broceato che Ludovico il Moro, nell'estate del 92. pagò in ragione di quaranta 


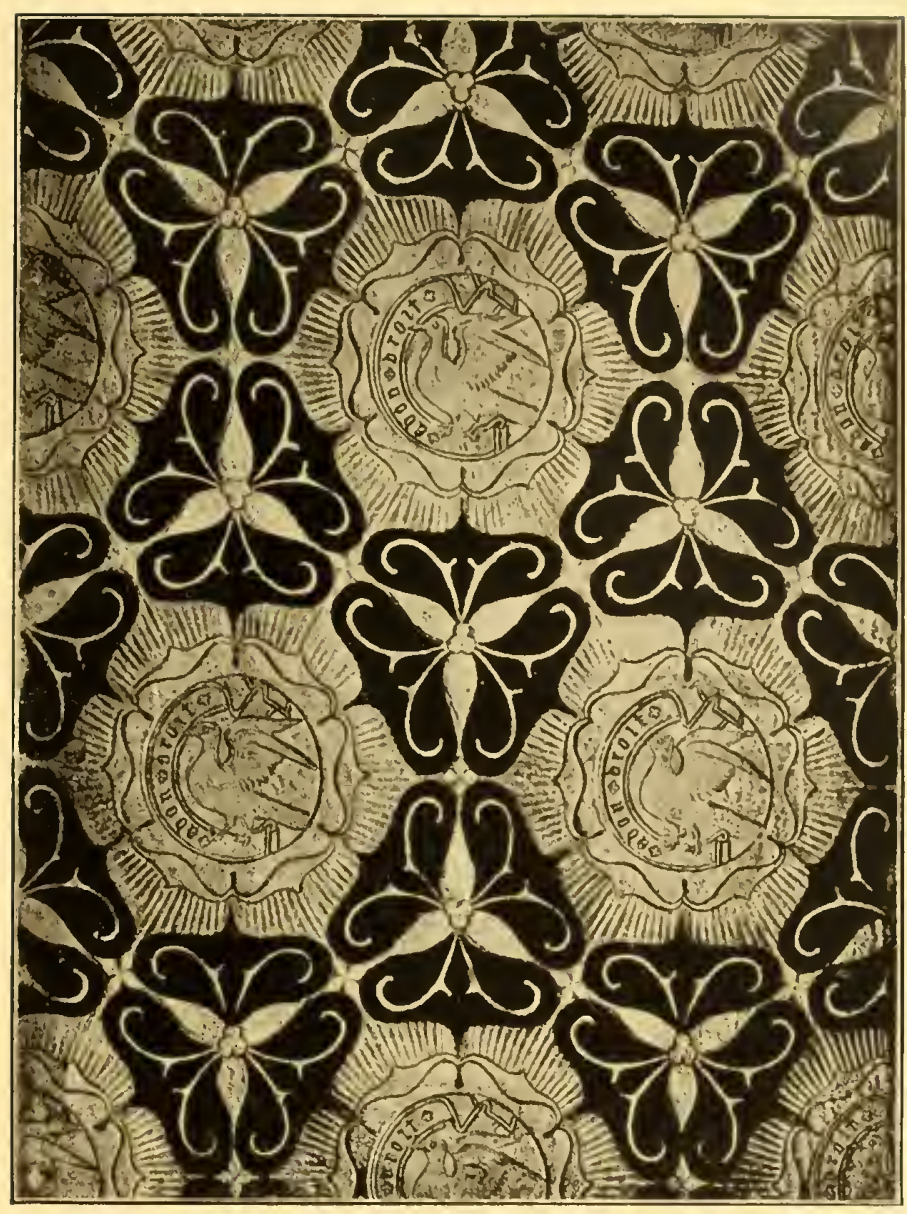

Fig. 8. - Velluto SForzesco (Museo Poldi-Pezzoli, in Milano).

ducati il braccio, per farme una veste a Beatrice consorte: era in "rize soprorizo d"oro cum qualche argento. lavorato ad una divisa che si dimanda el fanale, zoè el porto de Genua che sono due torre. cum uno bre. ve che dice: 'Tal trabalio mes places par tal thesauros non perder " (8).

Tale traffico mi piace per non perdere tanto tesoro: parole sehiette che rivelano inestinguibile sete di hosso, ostentate in una veste quasi per riconoscenza all'attivo commercio. fonte di tante raffinate dovizie e delizie!

La caratteristica tecnica negli enume- rati soprarizzi dioro e argenlo si eonferma illustre per destinazione, ritrovandola anche imitata dal pemmello di Hans Hohrein. con analitica precisione, in una veste indosso ad Enrico V'III d"Inghilierra. La quale veste ci fa appunto pensare a quel drappo menzionato imperiale d"oro in un trattato del secolo decimoquinto sul Larle della seta in Firenze, fra i piò pesanti a cari in ragione del metallo contemutovi (9).

Sebben fuori della tecnica fin qui seguita. dobliamo nondimemo, per il proprio stile aulico. annoverare alemaltre stoffe ancora. rifacendoci alla corte sforze- 


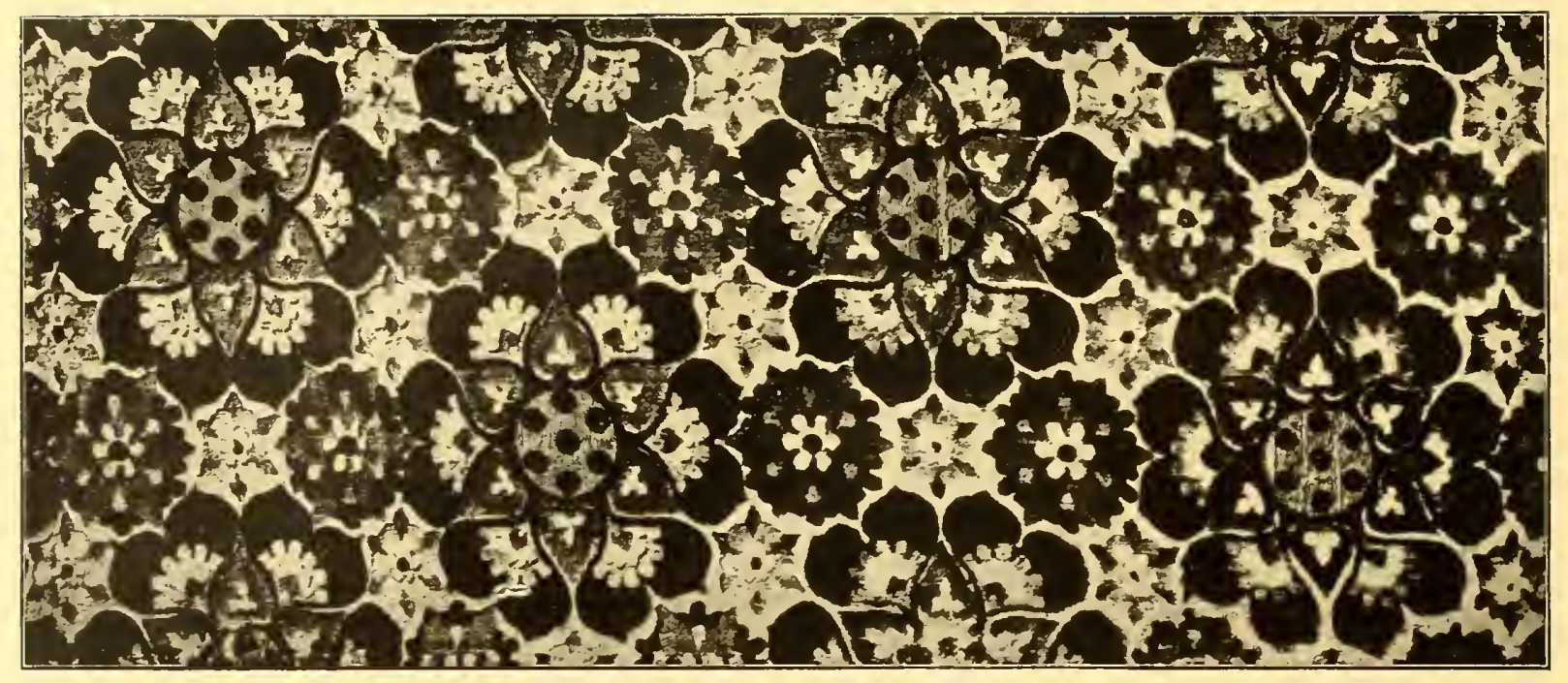

Fig. 9. - Velleto MEDICEO (Collezione Franchelti, in Firenzel.

sca. cui appariene il famoso velluto - ora nel Museo Poldi-Pezzoli di Milano - irradiato in campo rosso. di rosoni d'oro con le colomle in argento. recanti nu polizzino che dice: "A bon droit ) (fig. 8). Detto velluto. trasformato in paliotto per devota offerta. i forse quello stesso che Beatrice d'Este indossava in Venezia, quando fu ricevuta dalla Serenissima. al cui cospetto apparve. secondo una cronaca. " cum la veste de broccato d'oro de le columbine ) (10).

Son questi gli ultimi riflessi del lusso fantastico della corte di Ludovico, allorchè allarte del tessere si chicse il maggiore splendore. e fu la più intimamente amata come meglio consona al ginsto dei tempi. Nelle cronache di allora. i nomi delle stoffe preziose risuonano così spesso. e li accompagna lanta dovizia di ricordi, da trasformare alla nostra fantasia gli stessi episodii storici in rappresentazioni sceniche, dove gli attori illustri sembran mossi da una ricerca d'arte coreografica più che non da ragioni pratiche. E pur gli stessi misfatti che si vogliono attribuire al Moro. liberati col tempo dal. lorrore che ineutono, ci appaiono quasi intonato sfondo oscuro al rutilante sfarzo del suo mondo.

In Milano. se non solo per lui, ma certo per di lui impulso. Ia tessitura nel " 74 teneva occupati ai velluti hen quindicimila operai, le cui opere eran richieste avidamente dovunque. fin nella Germania. che mandava commercianti a farne incetta (11). c dove tuttavia insieme alle stoffe veneziane continuano a riflettere italico colore. dai sacri dipinti tedeschi ritraenti i più smaglianti aspetti del lusso ecclesiastico.

Ed ora l'esame di un altro velluto. ci conduce alla corte medicea al tempo del Magnifico (fig. 9); e palpita tutto di grandi rose dai petali simili ad ali di farfalle esotiche, disposti intorno ad uno scudetto doro a sette palle rosse. nella precisa forma di clipeo, qual’è segnato sul basanento donatelliano della tomba di Giovanni de Medici. in San Lorenzo.

Per lo stile più prezioso, proprio alle so- 


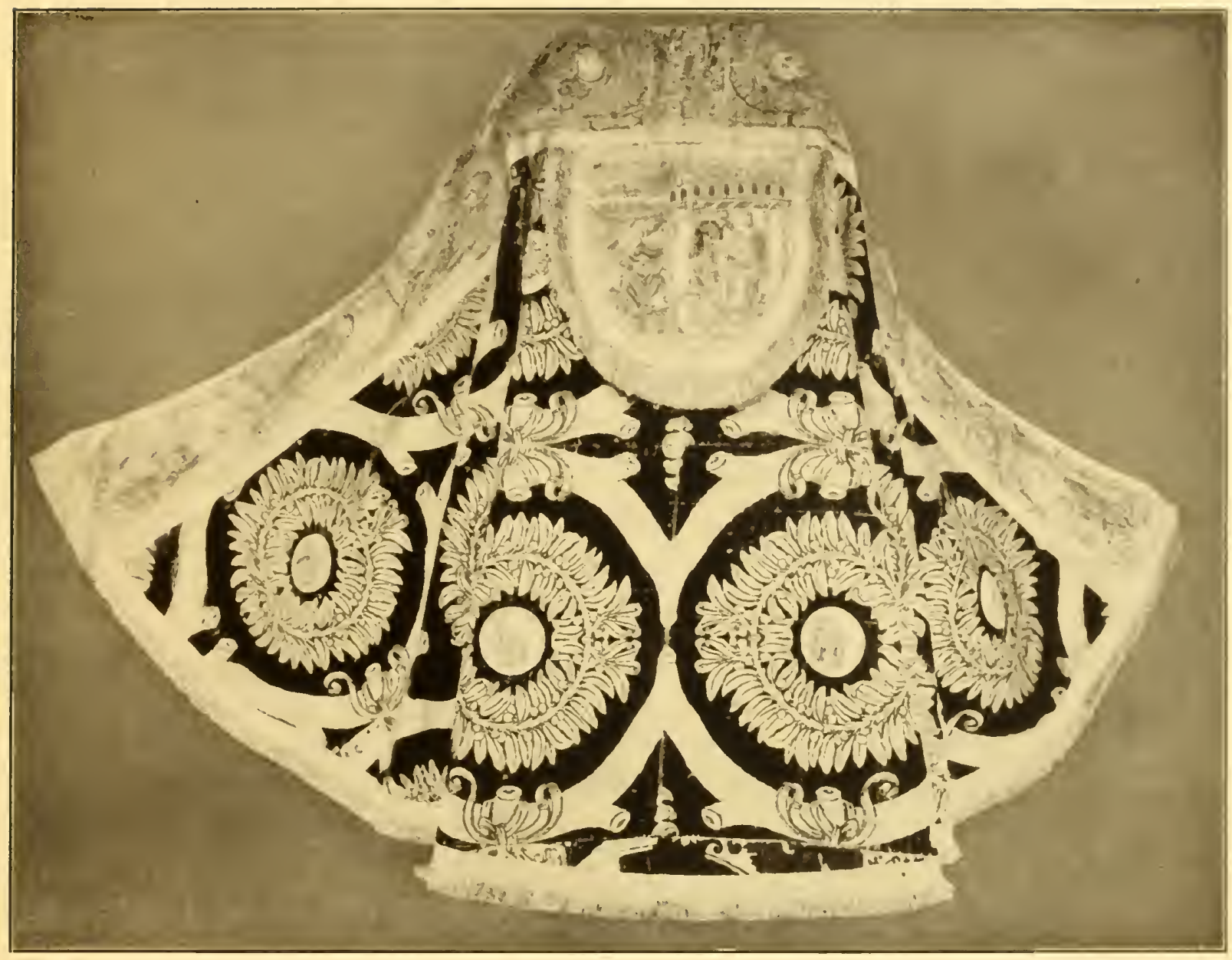

Fig. 10. - PIVIALE MEDlCEO (R. Galleria degli Arazzi, in Firenze).

lenni occasioni, si distingue il drappo pur mediceo. ond 'è confezionato un piviale, nella R. Galleria degli Arazzi in Firenze ( $f$ gura 10). In campo chermisi vellutato svolgesi d'oro un ampio disegno di rami al,binati senza fromla. congiunti da eleganti fiocelui. formando altstera cornice a serti di lauro, al eni centro: medaglioni con figure di Santi. rhe richiamano il ricamo del fregio e del cappuceio pluviale.

Secondo un frammento rivelatore nel Museo Artistico-industriale di Berlino - sotto ai medaglioni rieamati esisterebbero gli originali stemmi medicei: i quali offrono la particolarità araldica della settima palla grgliata ancora in rosso - co. me appena assmta allarma che comerva tuttavia la forma elipeata - e in maggior graudezza sulle altre, quasi si riferisse alloccasione delle nozze reali di Caterina de’ Medici con Enrico II di Francia nel 1533 (figura 11).

Di cotesti fasti diAracue medicea non meravigliamo pensando a qual punto di perfezione era giunta in Firenze l'industria tes. sile al quattrocento. Voeran nel 72 . secomblo afferma il Dei. ottantatre lotteghe magnifiche dellarte della seta, dinde la- 


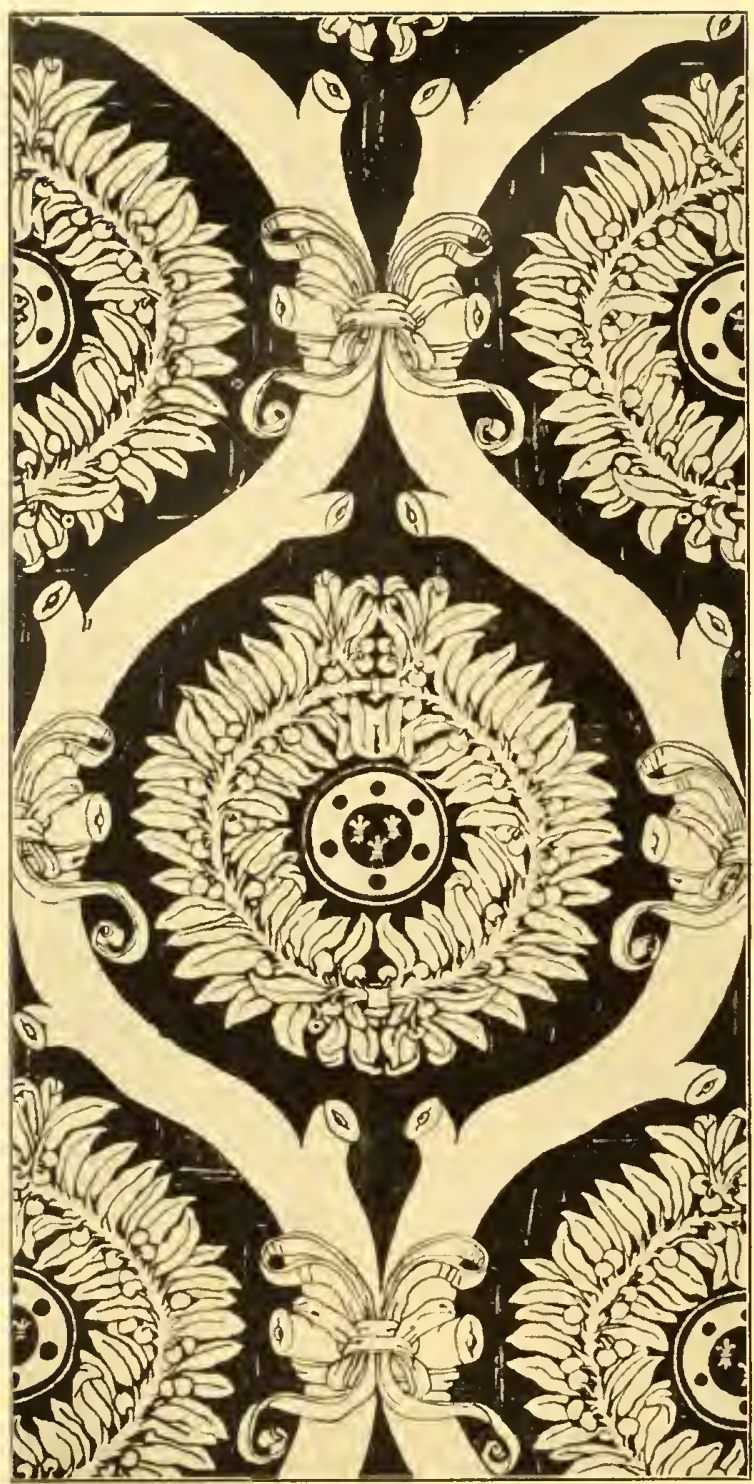

Fig. 11. - SVILIPPO DEL MOTHO PRELEDENTE.

voro a quindici o sedicimila persone, ocenpate a settemila telai battenti. dai quali fnivano tessuti ai mercati di Roma. Napoli. Genova, Spagna, Catalogna, Siviglia, Turchia, Barberia, Avignone. Londra, Lione e Anversa (12). Non ì fatta menzione di Venezia, che per conto proprio filava i colori dello spettro in mirabili armonie. nè di Milano sufficiente alle brame sforzescle; ma è ricordata bensi la Spagna stessa già cosi ricca, ed avida nondimeno di festosi drappi. ehe in misura di picche voleva prenderli alla bella Florencia, per mano delle proprie soldatesche gimnte all"Apparita: "Bella Florencia, tornaremos tus terciopelos a la medita de nuestras picas! ).

Ed ora si chinde la rassegna con lo stendardino figura 12. Ha il fondo broceato d'oro, ornato in velluto cremisi di due spessori con il consueto motivo del tronco fiorito: al cui centro campeggia un fiorone lobato a pennacchio e corolle intorno, recante intessuto uno scudetto in forma di bucranio, con leopardo gradiente, in riccetti d'argento, su prato fiorito. La solita cimosa ne limita i lati verticali, ed un listello taglia il disegno alle testate del telo. Lo stemma, non bene identificalile, potrebb'esser forse degli Alemanni, o dei Lopes che elbero un cardinale pontificando Alessandro VI.

Un quadro di Giovanni de Boceatis nella Collezione Cook in Richmond - rappresentante la Vergine col Bambino, mostra nn baldacchino con banderuole pendenti a disegno gotico e stemmi in mezzo: nıa pnò meglio darsi fosse il nostro un ornamento di qualche residenza o seggio, detti pancali negli inventarii del quattrocento, ove son descritti quasi sempre con armi e imprese, come in quello dei beni di Lorenzo il Magnifico.

Giunta a tal segno di perfezione da rendere con la ricchissima sua tecnica ogni libera fantasia artistica, lindustria gloriosa dei tessuti doveva quasi necessariamente avviarsi al decadimento. Li autumnale dorata ricchezza dei broceati e dei palii carichi di carli e di melagrane aperte, già ne segna la piena maturità; e negli anni che seguono il cinduecento, cominciano a cadere le bel- 


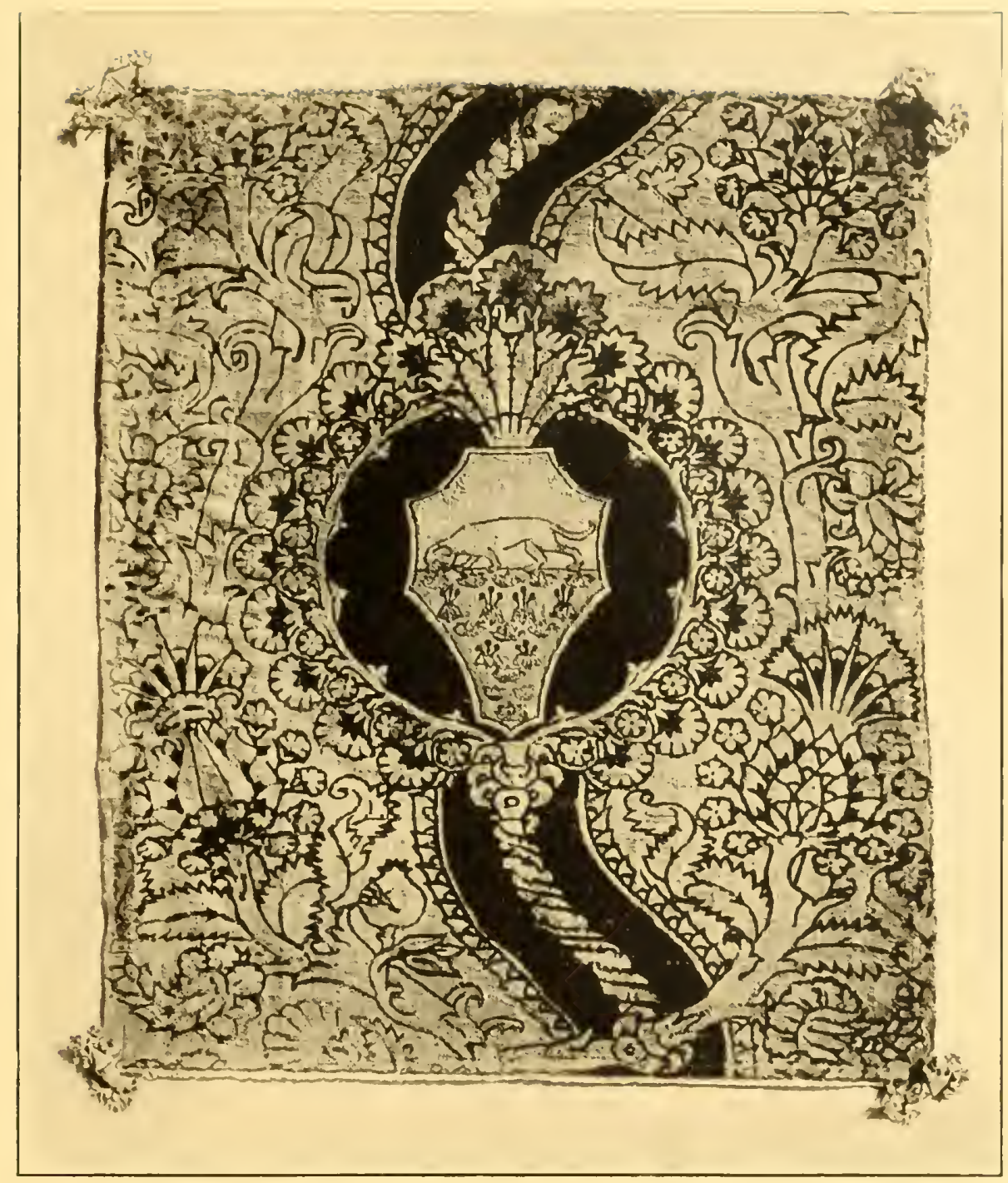

Fig. 12, - PA.XCALE GE.TTLIZIO (Collezione prisala).

le foglie lohate dalle tessili spalliere. seoprendo la geonetrica monotonia degli in-

(1) II paliotto di Sisto 11 tella Busilica d Assisi, ne L'Arte, 1906, III.

(2) Kunstgeschichte der Seidenweberei, Berlin, 1913, vol. II, pag. 114, fig. $5+6$.

(3) Riferito in: Archeologiai Ertesitö, 1887, pag. 404, e in Millouiums terkes, 1896, vol. II.

(1) MUNTZ, Les Arts e la Cour des Papes Innocen! I'III, Alexandre II, Pie III, Paris, 1898, pag. 128; 1.

S. Y., Intr. et Ex., 1489.1490, fol. 220.

(5) Ibillem. p. 121: A. S. V.. Divers. Cam.. I189-It9], ff. $278,279 \mathrm{~V}$.

(6) J I.ESSING, Die Schuester des Preussisschen Kron. tricali arbusti, che non daranno piin fori nè frutli per un lumge inveruo.

tresor, in: Jalubuch der K. Preas. Kunst., 1895, pagina 120 .

(7) MUNTz, op. eil., pag. 39; 1. \&. V., lutr. el Ex. 1489.1190, fol. 218.

(8) MALAGZZZ1, La Corte di Lodovico il Moro, vol. I, pag. $4 \mathrm{~J} 0$.

(9) Per cura di G.IRGiolLI, el. Barbèra, 1868, cap. L e L.XVI.

(10) MALAG(ZZ,, op. el vol, (ill., pitg. 410.

111 Ibident, pagg. 16.5 a 168.

$112)$ GARGIOLLl. op. cit.: Comeuto al Trattato, pa. gina 225. 



\section{RELIQUIE TESSILI RINVENUTE NELLA TOMBA DI SIGISMONDO PANDOLFO MALATESTA IN RIMINI}




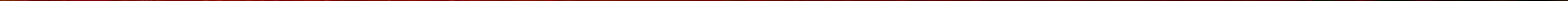




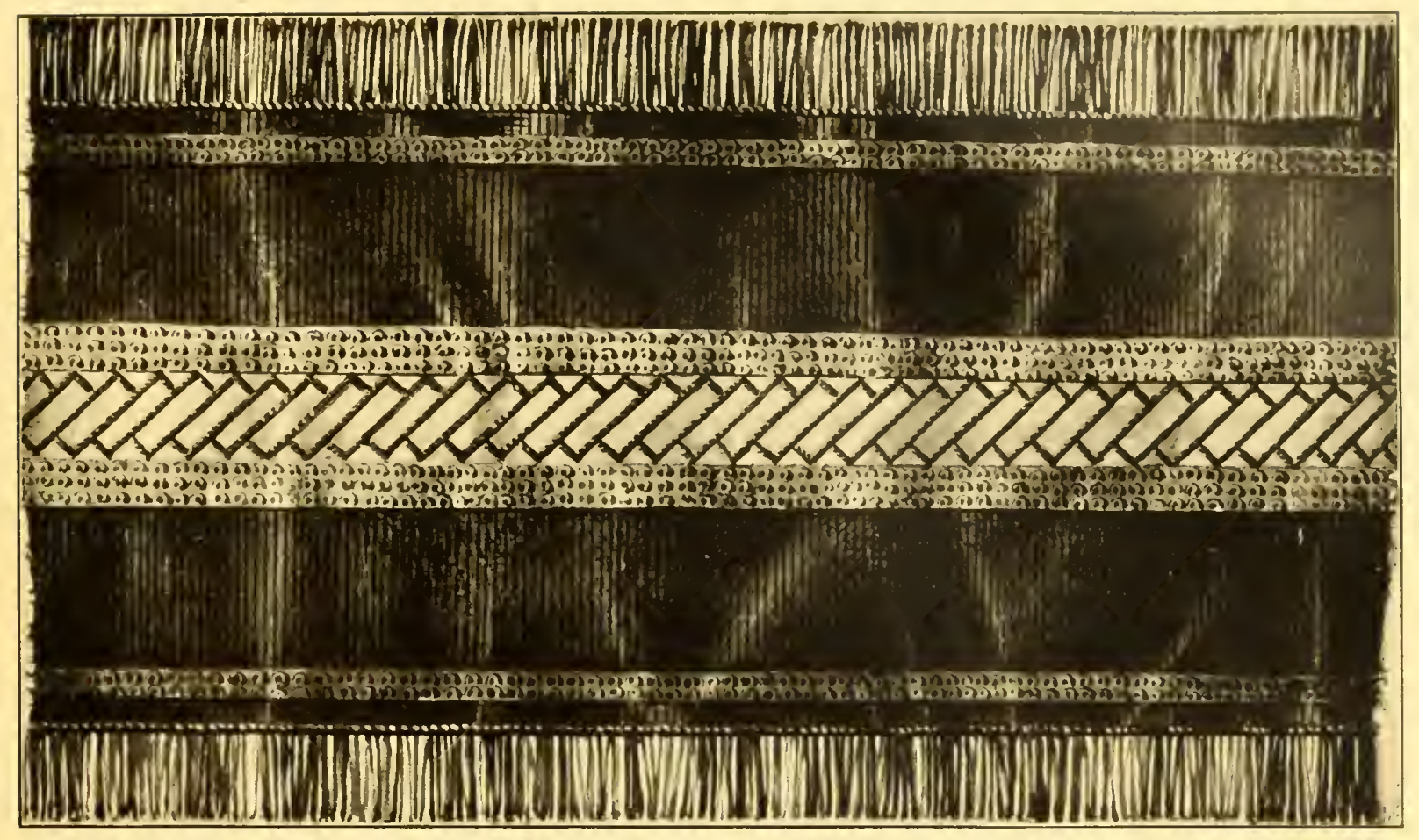

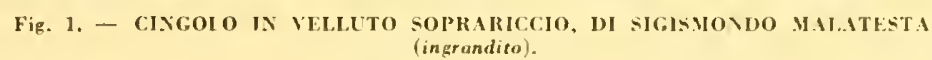

\section{RELIQUIE TESSILI RINVENUTE NELLA TOMBA DI SIGISMONDO PAN- DOLFO MALATESTA IN RIMINI (*)}

Intessute nei secoli dogni nostra virtù e difetto. dallinnato pudore alla decadente vanità. le stoffe, quali necessari prodotti artistiei, sono tenacemente comnesse, sebben voluhili negli aspetti, alla vita e alla storia dellnomo, non meno della veste vellosa o pinmata a certe belle specie animali eni ne rivela le abitudini e il carattere.

Eeeo perchè quando evochiamo un dato momento storico si illuminano alla nostra fantasia, esperta nei segreti del passato improvise immaginazioni tessili balenanti e rolubili secondo il mutare de" tempi. Basta

(*) Datla Rassegna d'arte, Marzo, 1921. talvolta un solo nome: nominiamo Costantino. e un subito fruscio serico. In hishiglio di sommessi colori si desta dalle ciciate porpore della corte di Binanzio a chinderci se dobhiamo intendere il Porfirogenito, o non invece il suo sciallo omonimo togato. recante il labaro di Ciristo alla soglia del medioevo. Tale si compie l'estetica della storia uello studio dell'arte testrina.

Taceio quanto ancora palpitasiero le stoffe alle aure del Quattrocento, perehe lo dicono pure i marmi. ( in ispecie puelli del Tempio Malatestiano di Rimini: il puatle. come dedicato in segreto all amore di Sigismondo per lsolla. mentre si presonta 


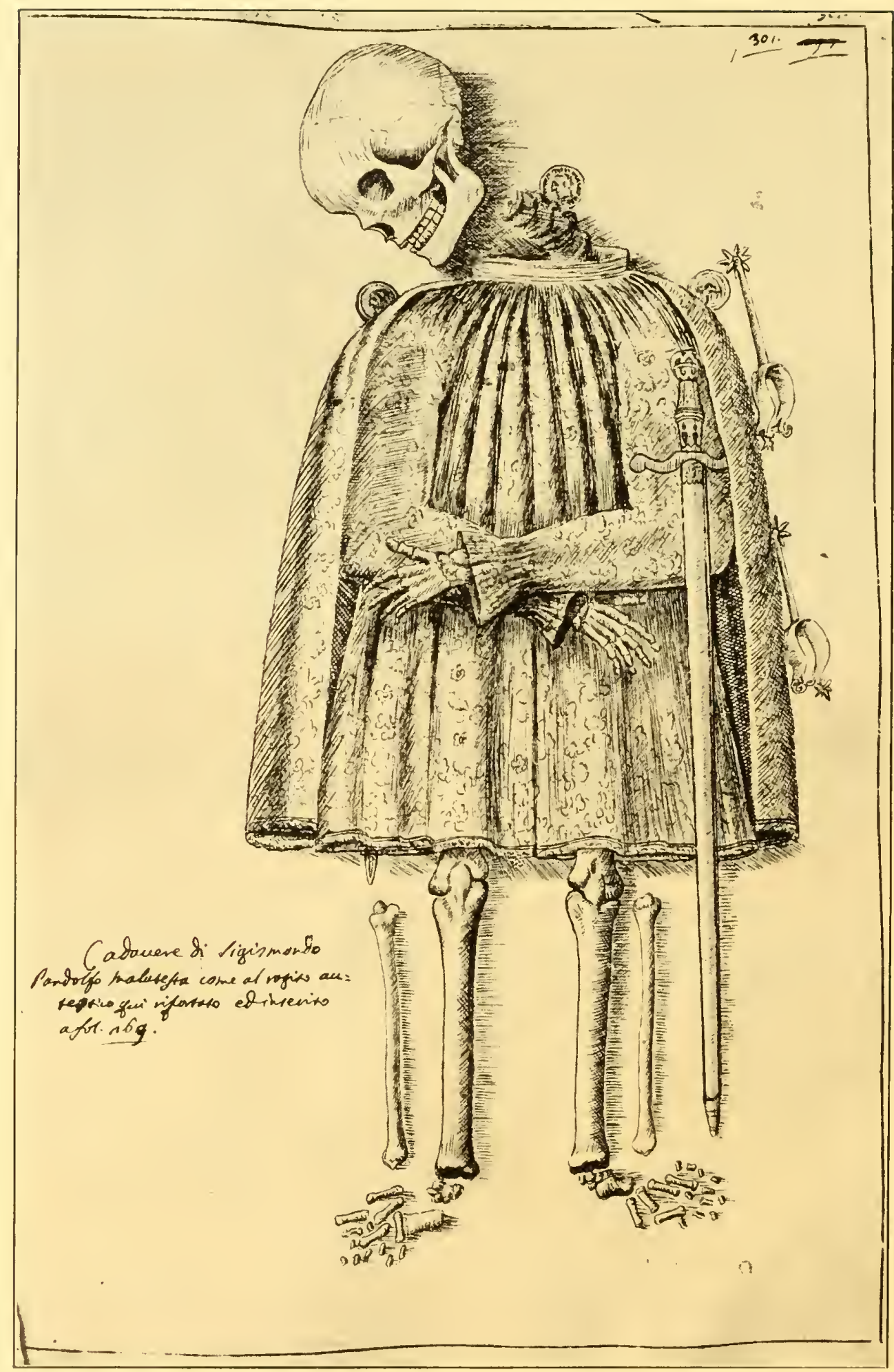

Fig. 2. - RELIQUiE TOMBALI DI SIgisMONDO MALATESTA. QUALI SI RINVENNERo Nel 1756 (Da un'incíbione in "Novello Lellerarie 》). 


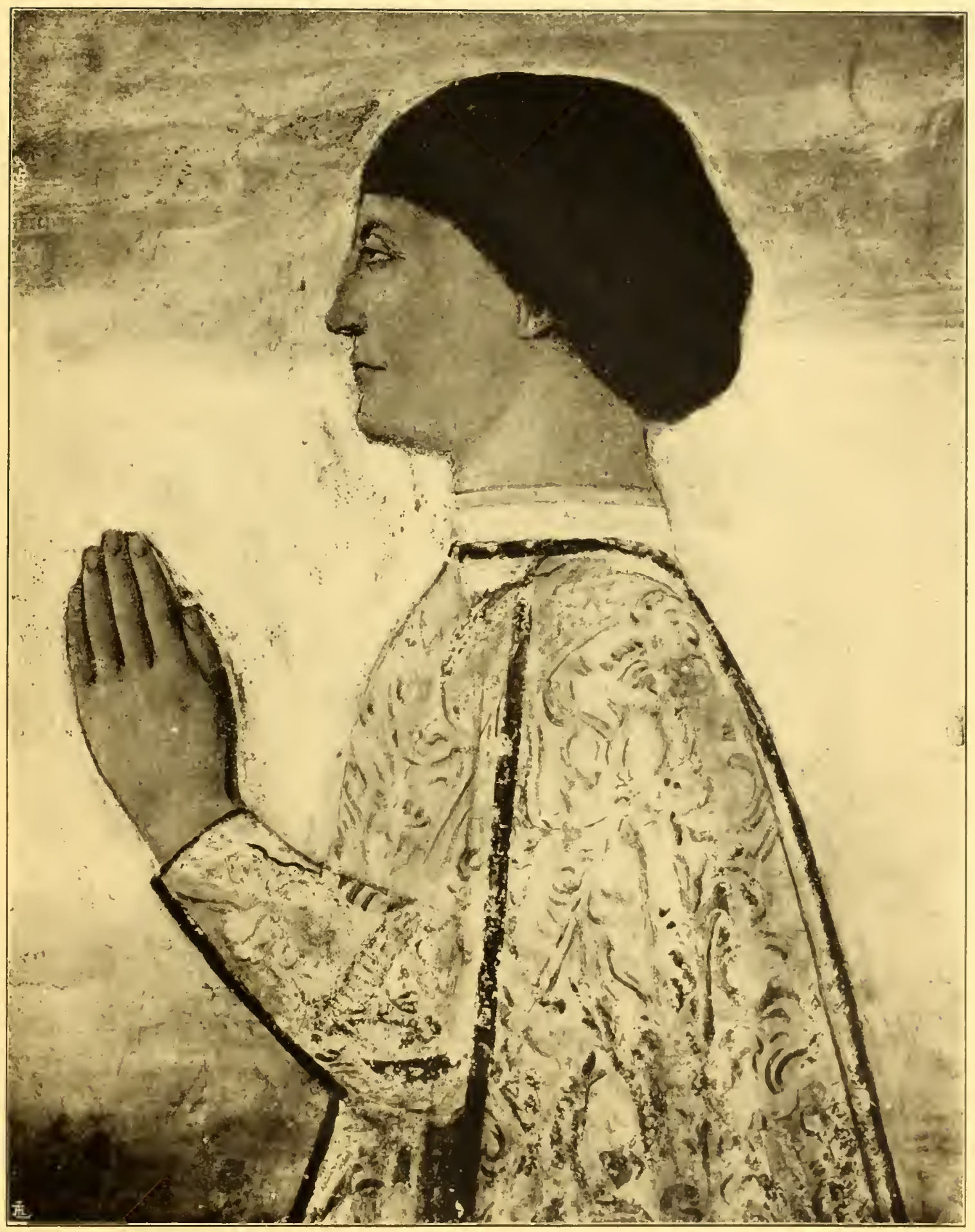

Fig. 3. - Rithitto di sicismondo P. MALAtesta (Pier della frunceaca). 
al di fuori logicamente freddo nelle sue unanistiche linee albertiane, dentro conserva del romantico tepore medioevale fra gotici drappeggi d'alcova pictrificati in armonici accenti al sacro silenzio delle tombe illustri.

Contemplando appunto quella che n`è priva, di Sigismondo Pandolfo Malatesta. allogata presso la cappella del di lui Santo patrono, spesso lo sguardo della fantasia, stanco d'indugiare fra nonotoni chiaroscuri architettonici, si spinse entro l'arca cone a ritrovarvi qualche lembo di colore che ben sintonasse al magnifico condottiero cui furon pur tanto care le stoffe, quali un suggestivo complemento d'imperio richiesto dai tempi suoi.

Ma oggi alla luce del soguo. si contrappone la realtà con la propria conseguente ombra di delusione: delusione - dicjamolo subito - dovuta al tempo operante in diuturno silenzio nell"oscurità dell'avello dal 1468, in cui morì Sigismondo, fine all'odierna ricognizione, avvenuta il 28 Settembre ultino scorso, per iniziativa di Corrado Ricci. proprio in punto a salvare dallestremo dissolvimento quanto nell"arca insigne ancora rimaneva.

Rimossovi il coperchio si poterono individuare. con i resti mortali miseramente confusi, varii frammenti tessili, le parti disginnte della spada e del pugnale, gli speroni ed alcune medaglie di Matteo de' $\mathrm{Pa}$. sti ( l).

Causa del disurdine fu ma precedente incauta verifica nel 1756, alla quale dobbiamo in compenso un verliale abbastanza preciso ed olviettivo. che ci aiuta a ricostruire la foggia del vestito, di cui alcu. ni brani. fra i meno corrotti e raggrinziti, mostrano ancora il loro disegno nelloriginale aspetto (2). Ma prima di procedere all'esame diretto delle reliquic, cominciamo col leggere i passi del verbale che ad esse si riferiscono. tenendo sott'occhio $\mathbf{j}$, lisegni, che, sulla scorta del vero, furon tratti allorquando ogni cosa stava in sito. c l'opera del tempo non era ancora irremeahilmente avanzata ( fig. 2) (3).

"Questo cadavere [quello di Sigismondo] era vestito di un ferraiolo cadente dalle spalle, che arrivava fino alla metà delle coscie; e questo di un drappo fiorato d'oro con fondo color tané, allorlo del ụua. le stava una frangetta d'oro e seta del medesimo colore, larga poco meno di un dito: quali fiori, a ricamo d'oro, erano di struttura piuttosto grande. Col detto ferraiolo stava coperto per la metà del corpo alla parte sinistra, quale rimossa, si videro le mani, e si osservò avere un abito grossa. mente increspato di simile broccato fiorato d'oro con fondo di eguale colore tané; e questo si estendeva sino alla linghezza del suddelto tabarro. e alliutorno portava una frangetta come sopra d'oro e seta slel me. desimo colore; il quale abito era foderato di taffetà di colore suddetto ed aveva le maniche non molto larghe. ma lunghe in modo che arrivavano fino ai polsi di aubo le mani, e sopra di essi sincrespava alluso dei manichetti; nel resto era fatto a foggia di rocchetti dei preti, sciolto ai fianchi. aperto per altro davanti. co suoi fori dal. lina e laltra parte. come se da una stringa allacciar si dovesse. la quale però non ostante la somma diligenza usata non fu per verun conto veduta e trovata. Il modello di 


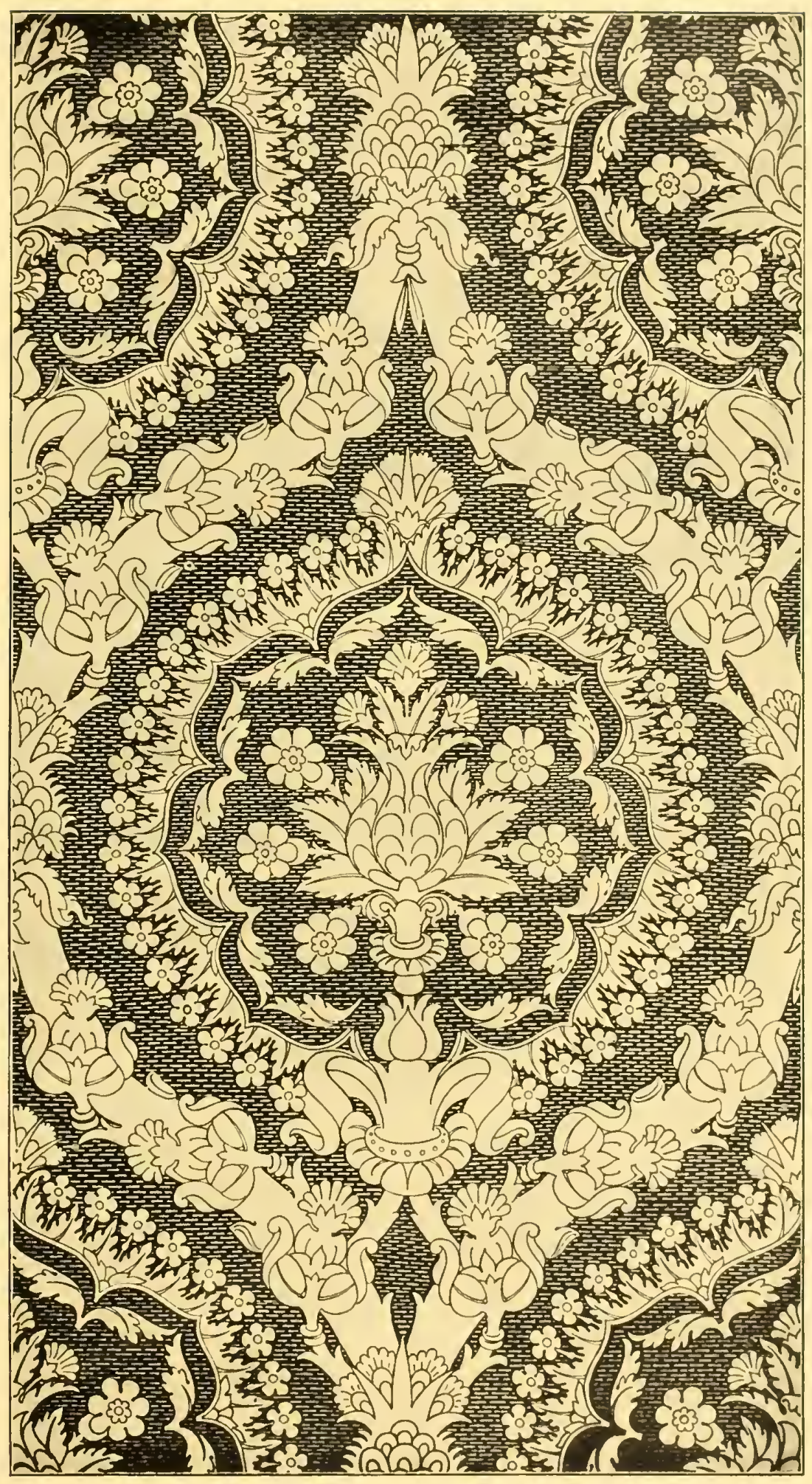

Fig. 4. - BROCCATO DEL MANTELLO DI SIGISMONDO MALATESTA 
questo abito può eiascuno che desidera vederlo nella pittura o sia ritratto del mede. simo Sigismondo Malatesta. che mirasi ne] muro interno della Cappella detta delle reliquie, sopra la porta, dipinto un tempo da Pietro di Borgo Sansepolero l'anno eioc̀ di nostra salute 1451 ).

I pezzi rimasti. poco agginngono alla supposta forma originale del deseritto ferraino. lo o giornea: il maggiore conserva ancora le increspature pertinenti allo scollo; altri minori reeano la loro frangia eucita : $1 l$. balza inferiore; e molti brandelli anorfi derivano dalle parti intermedie. Nondinirno nulla coniraddice al raffronto con l'affriseo di Pier della Francesea, ove chiaramente si vede il mantello, conginnto ad una specie di giobloa, cadere dalle spalle ad ampie picghe verticali ( fig. 3). Era del resto quest'indumento molto in voga nel medio quattrocento, e variando di poco nel taglio nella lunghezza, lo ritroviamo spesso in altri ritratti di cavalieri e personaggi cosyricui di allora.

In quanto alla stoffa cui era fatto, erro. neamente ritenuta in ricamo, è invece di broceato d'oro (4) su fondo rasato viola, ehe dalla decomposizione eadlaverica lıa preso del bruno paonazzo, esattamente definito color tané (5). Il suo disegno appar quello solito a cardi, posti entro una gran foglia lobata florescente, intorno alla quale sincurvano rami potati e conserti a formare scomparti ammandorlati (fig. 4).

Siffatto motivo s'identifica fra le sue tante riproduzioni pittoriche, nel piviale di un Santo adorante il Bimbo in grembo la Madonna, opera del Signorelli, nella Galleria Uffizî. Però alla nostra stoffa il fondo è cosparso di lineole d'oro, secondo una moda che anche dai dipinti che la docu. mentano dobbiamo giudicare comune in Firenze alla metà del secolo decimoquinto, come per esempio dall'affreseo di Benozzo Gozzoli nella Cappella di Palazzo Riccardi (1460); il quale affresco mostra un re magio vestito d'un drappo tutto vergolato, che cosi chiamavasi allora, mentre a due cammini sintendeva il doppio movimento divergente e convergente degli steli salienti. Stereotipo nello sehema, sebbene infinitamente virio nei particolari, codesto il tipo ehe intitoliamo malatestiano dall'esemplare di Rimini, e di eui diamo nu saggio consimile nel broccato purpureo fig. 5 .

"Aperto il sopradeseritto abito - sęrue il verbale - viddesi di sotto un corpetto. non foderato, con maniche, corto però ehe soltanto arrivava fino all'inguine, stretto alla vita; e questo di velluto tagliato con soprariccio d'oro, con piccole asole da ambo to parti, e piccolissimi bottoni fermati sopra la destra parte, e questi fatti della stessa roba ).

Del velluto di questo corpetto, a merlio farsetto, si sono potuti riconoseere solo poehi frammenti induriti e contratti come suola brueiata; aleuni dei quali mostrano ancora le loro asolette a sopragitto ben fitte e gli ocehielli cerehiati di ottone ossidato. Le maniche, mella medesima stoffa, si presume uscissero dal ginbbone di broccato, di sotto al ferraiuolo, nella guisa dei nostri vecehi pastrani da sera detti pipistrelli.

Il verbale eschude fosse il farsetto fode. rato, ma con un più attento esame potemmo scorgervi presso le costure residui di tela che indicano il contrario. Della fodera di taffetà del mantello, notata nella prima ri- 


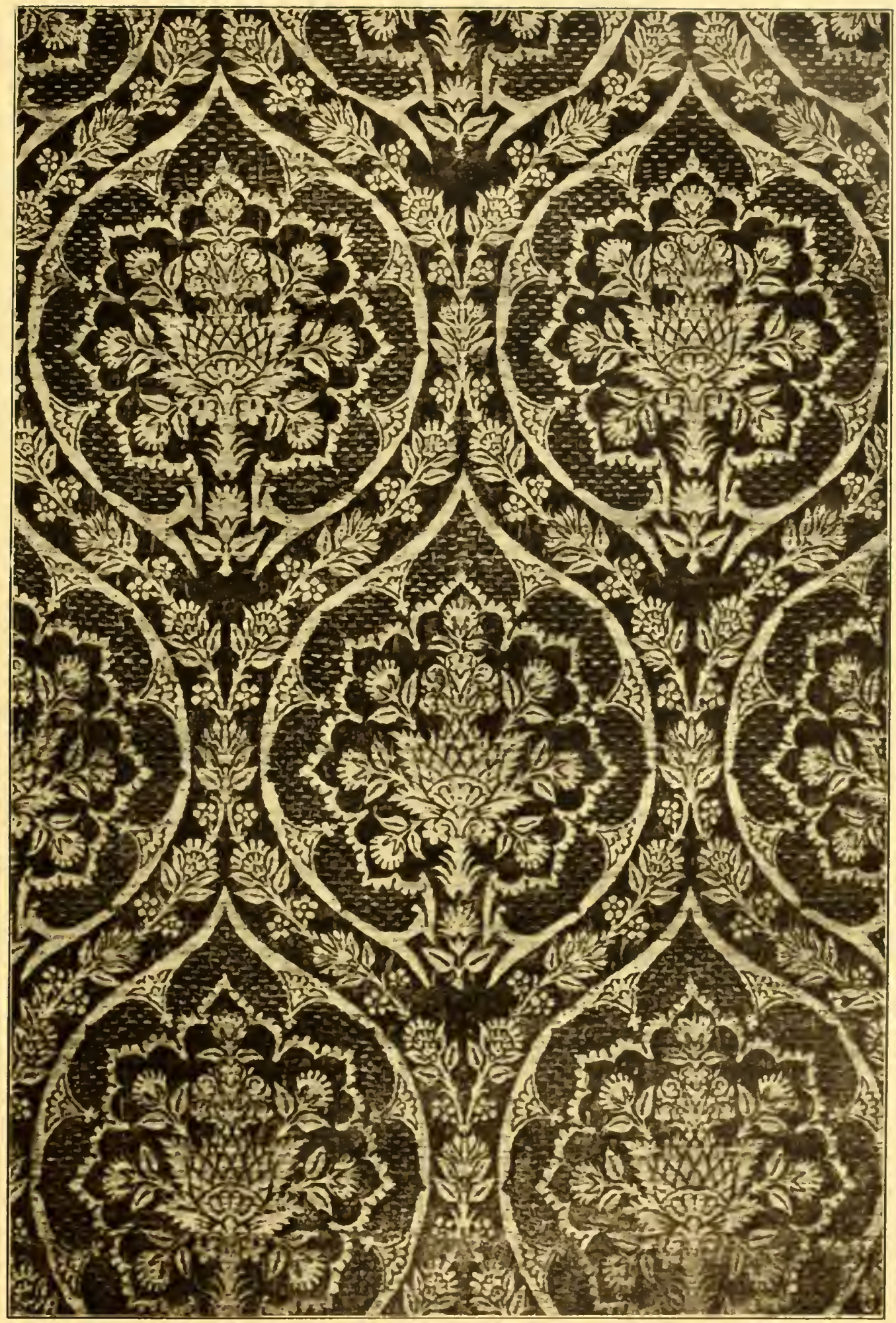

Fig. 5. - BROCCATO NORO DEL MEDIO OI'ATTROCENTO 


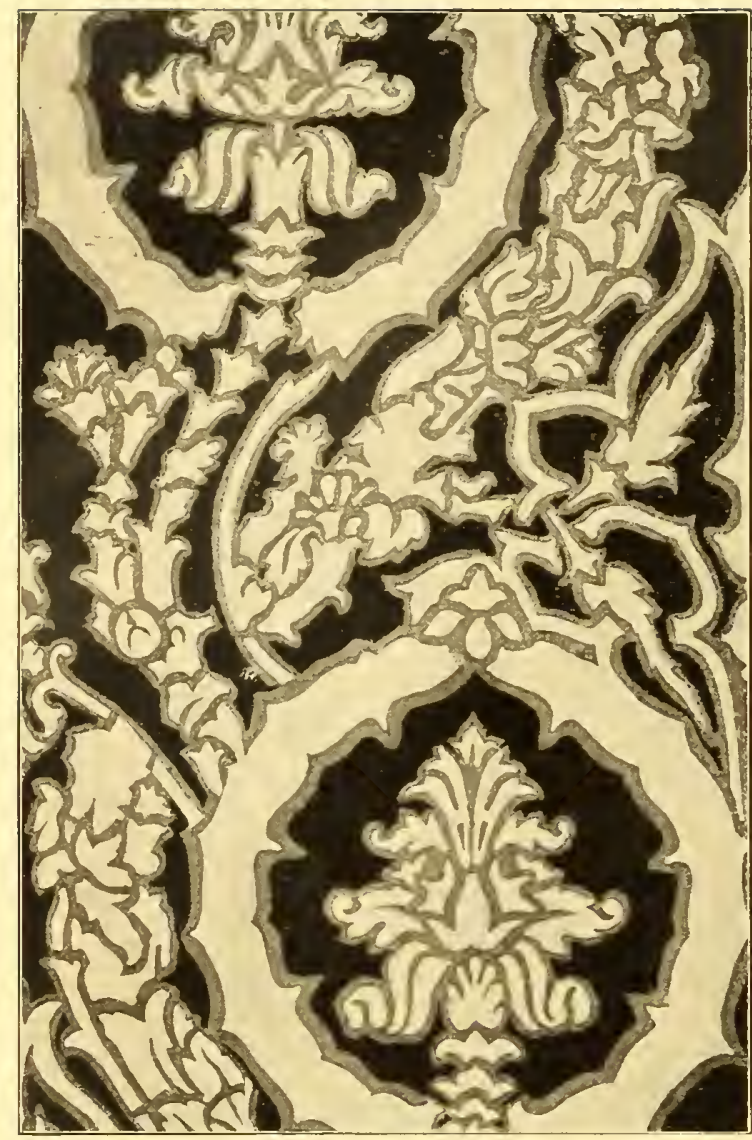

Fig. 6. - DECORAZIONE PARIETALE NEL DLONO MALATESTIANO.

cognizione, non rinangono invece nenmeno le tracce. Abliamo, per altro, vestigia d"imbottitura bambagina. che, giusto il Marchiello, crediamo avesse servito a riempire il petto e a far più svelte le reni (6), come si vede nei pettoruti ritratti di Sigismondo in sulle medaglie e nell’affresco del Duomo.

Il drappo di corlesto indumento risulta anche a noi di velluto tagliato con sopraric. cio doro, e le minime parti che ne rimangono consentono identicare il tipo del motivo con quello dei velluti dipinti in viola e oro sulla parete della tomba di Isotta e nelle ogive della cappella a riscontro ( $f$ gura 6); e pur esso doveva essere purpureo in origine, secondo mostrano i lembi ribaditi. Il disegno ampio e mosso, quale si legge sulle pareti c sinterpreta nei resti originali. ricorda assai da vicino certi cartoni tessendi tracciati dal Pisanello nel Codice Vallardi; per cui non ci sembra troppo ardita ipotesi. che l'autore di alcune fra l. più helle medaglie malatestiane siasi degnato anche ideare gualche ricco drappo come questo, ad allietare e la cortc e il tempio e la persona stessa del suo signore.

All immaginare più precisamente quale dovettessere detto velluto soprariccio prima che il tempo lo deteriorasse. ci soccorre IIn felice caso che ha voluto farcene conoscere un maggior frammento in migliore stato di conservazione. ma ginnto a noi per diversa via (fig. 7).

Quest iucontro non meraviglia quando si pensi che da un solo telaio uscivano stoffe a metri; le quali, soggette al facile taglio delle cesoie, ancor oggi si riscontrano di quando in quando come le stampe da un rame comme: onde molte edizioni tessili sono per rarità conosciute dagli studiosi con criterio, che direi filatelico. Si tratta dunque di un velluto eguale nel motivo e nel colore a quello del farsetto, e identico nella tecnica. avendo entrambi lo stesso numero di duplici fili d'oro per ogni centimetro quadro. e la stessa distanza fra i cappietti del soprariccio; dati intrinseci che confermano ai due pezzi una manifattura comune, con l“istessa certezza che offre alla polizia scientifica il raffronto delle impronte digitali.

Sequita il verbale: "Cingeva ai lombi. sonra il descritto corpetto. una fascia larga tre buone dita, che ai suoi capi aveva una fiblia grande proporzionata al cingolo. e 


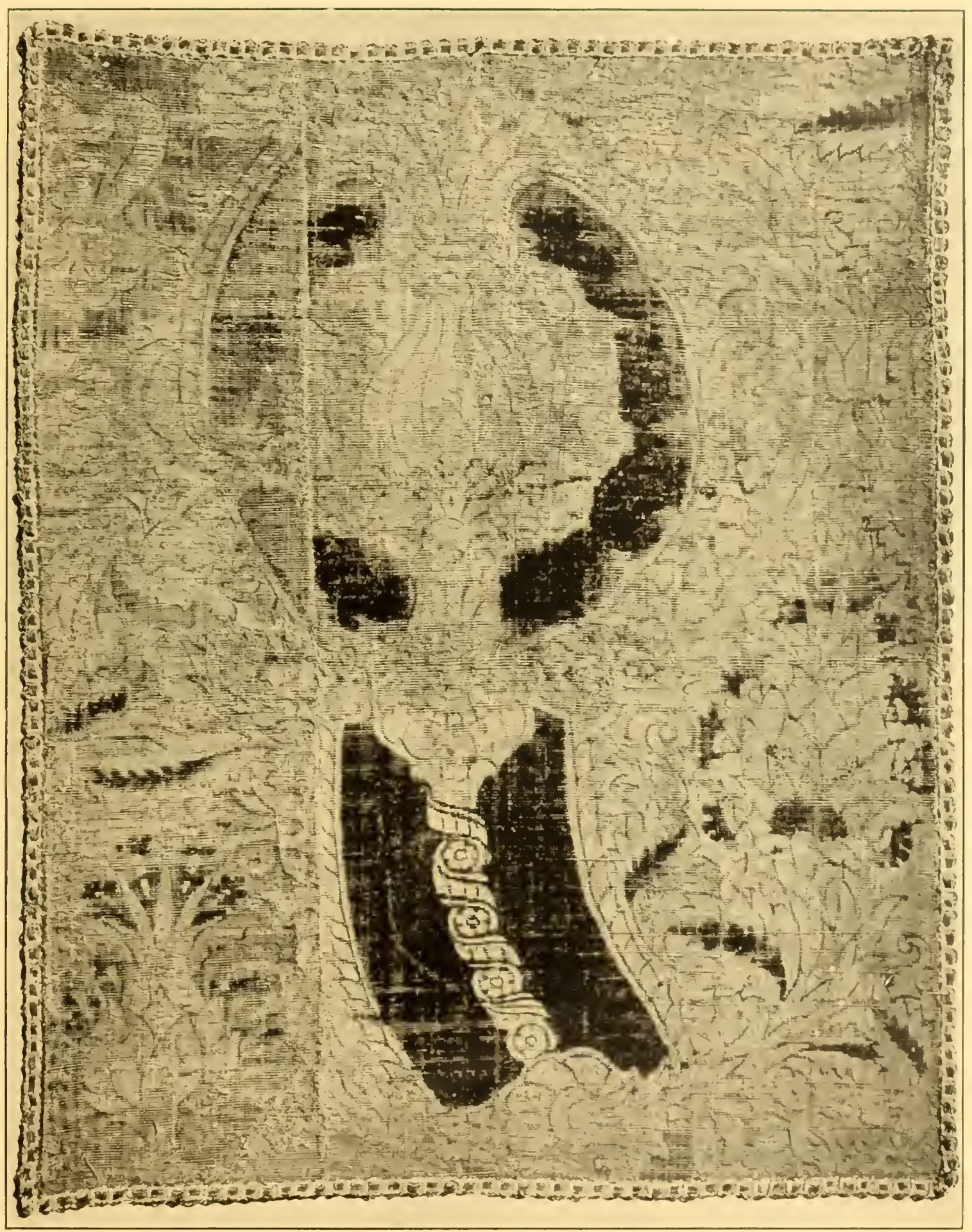

Fig. :. VELLUTO SOPRAIICCIO DI ORIGINE MALATESTIANA 
questa di metallo dorato; era il cingolo suddetto fatto di un passamano assai grosso in. trecciato e ben ricco d'oro con frangia da ambo le parti di larghezza circa un dito di seta e oro di color tané suddetto ).

Anche qui la descrizione collima con quanlo abhiam potuto osservare nei pochi tratti residui del passamano. se non che looro n’è ormai ossidato del tutto e la frangia in parte caduta. Solo rimane a precisare il genere del tessuto, ch`è di velluto viola intramezzato di soprariccio, e percorso al mezzo da 11 apposito radore con fregetto spirale per dare adito all'ardigione della fibbia e fissarne il passo ( $f g$. l). Da detto cingolo - secondo il verbale - pendea lo stocco.

Della camicia rimangono pochi lacerti di tela alquanto grossa, di cui uno aderisce ad un segmento di lamina metallica dentellata che richiama la guamizione del fodero della spada: oude si può supporre pertinente ad una cintura indossata fra la camicia e il corpetto, alla quale appunto sarebbe stata assicurata la stessa spada.

Altri pezzi di tela, per essere incorporati a calcinacci, caduti forse murando la pietra

(1) Di ogni cosa ritrovata darà contezza Corrado Ric. ci nella sua opera Il Tempio Malatestiano; intanto noi, pre di lui cortese invito, anticipiano la pubblicazione delle stoffe come quelle che più interessano ai nostri studii.

(2) L'atto di ricognizione, in data 21 Agosto 1756, fu registrato dal notaio Francesco Antonio Masi, e trovasi traseritto nelle pagine 268.271 del Campione del Con. vento di S. Francesco di Rimino del Padre Francesco Anton Maria Rigluni, volume manoscritto di proprietà del Dott. Carlo Piancastelli, in Fusignano. sigillare, crediamo poterli attribuire a 111 supposto lenzuolo funehre.

Delle calze o panni di gamba, che erano in genere di saia, non rimangono tracce dal tempo della prima ricognizione, e ciò si spiega facilmente per la materia lanosa quale più corruttibile e in più diretto contatto col corpo.

Spariti dunque in progresso di tempo e in ragione di resistenza igrometrica. prima le saie, poi i lini e le fodere seriche, restano in parte $i$ più preziosi broccati che nel loro relativo buono stato han già perduto se non l'ordine molecolare, la flessiluile coesione, onde irrigiditi cedono in polvere al menomo contatto. A ritardarue la fine occorrerebbe chiuderli ermeticamente entro una bacheca con speciali vetri fluorescenti che li difenda dall’aria e dalla azione chimica della luce. più deleteria.

Intanto noi abbiam voluto salvare dal tempo almeno la nemoria di codesti drappi; i quali, se nulla di nuovo dicono alla storia dell'arte tessile, aggiungono una realistica pennellata di colore al ritratto fisico del magnifico capitano che li indossava.

(3) Riferiti in Norelle letterarie di Firenze, volume XV11I, pag. 262.

(4) Ora completamente ossidato, onde scopre l'anima di flo che biancheggia qua e la, traendo in inganno sul colore cli non tenesse couto delle alterazioni dovite al tempo.

(5) Anche nelta Relazione dapertura davelli, in Novelle letterarie, è avvertito il color viola del manto.

(6) MERKEL, Come vestivano gli uomini del Deca. merone, Roma, 1898, pag. 16. 


\section{TESSUTI FIGURATI FIORENTINI}




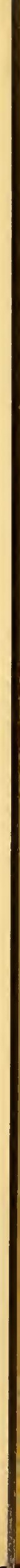




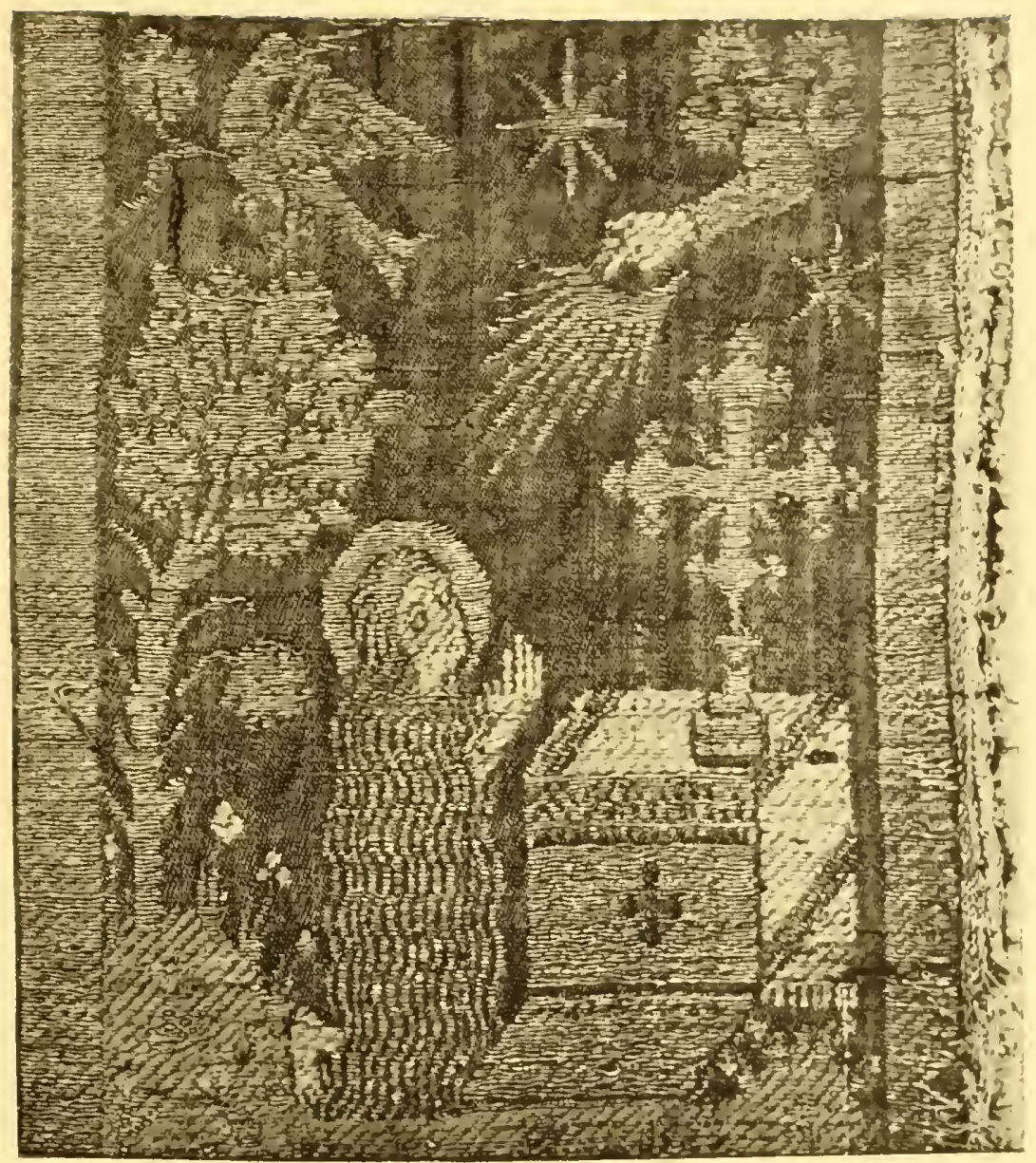

Iig. 1. - TF.sslto LitLRgico GIOTtesco.

\section{TESSUTI FIGURATI FIORENTINI}

Quando dopo la morte di Manfredi, la scuola poctica siciliana dovette migrare nel. le città di Toscana e di Romagna, lindustria tessile, fino allora monopolio palatino, per non estinguere con la corte sveva, si shandò pur essa, trovando stanza in Lucea, ove elobe agio prosperare nella feconda libertà degli aperti commerci.

Come vi gimse era tutta carica di motivi

(*) Da Dedalo, anno II, fasc. III. arahici e di risonanze hizanline: ma nel nuovo ambiente. in contatto dello stil nuo. vo, che fu primavera ancle alle arti figura. tive, comincio hen presto a modificarsi: i smoi tipi animali, pur tultavia vivaci. perdettero quel proprio aspetto belluino e ehimerico; $i$ fori e le piante ne divemero pii verosimili, e le zone di lunghe iscrizioni arabiche diedero luogo. prina alle funte legrgende enfiche, quali ci mostrano le Madonue primitive dai loro veli, poi alle latine e ita. 


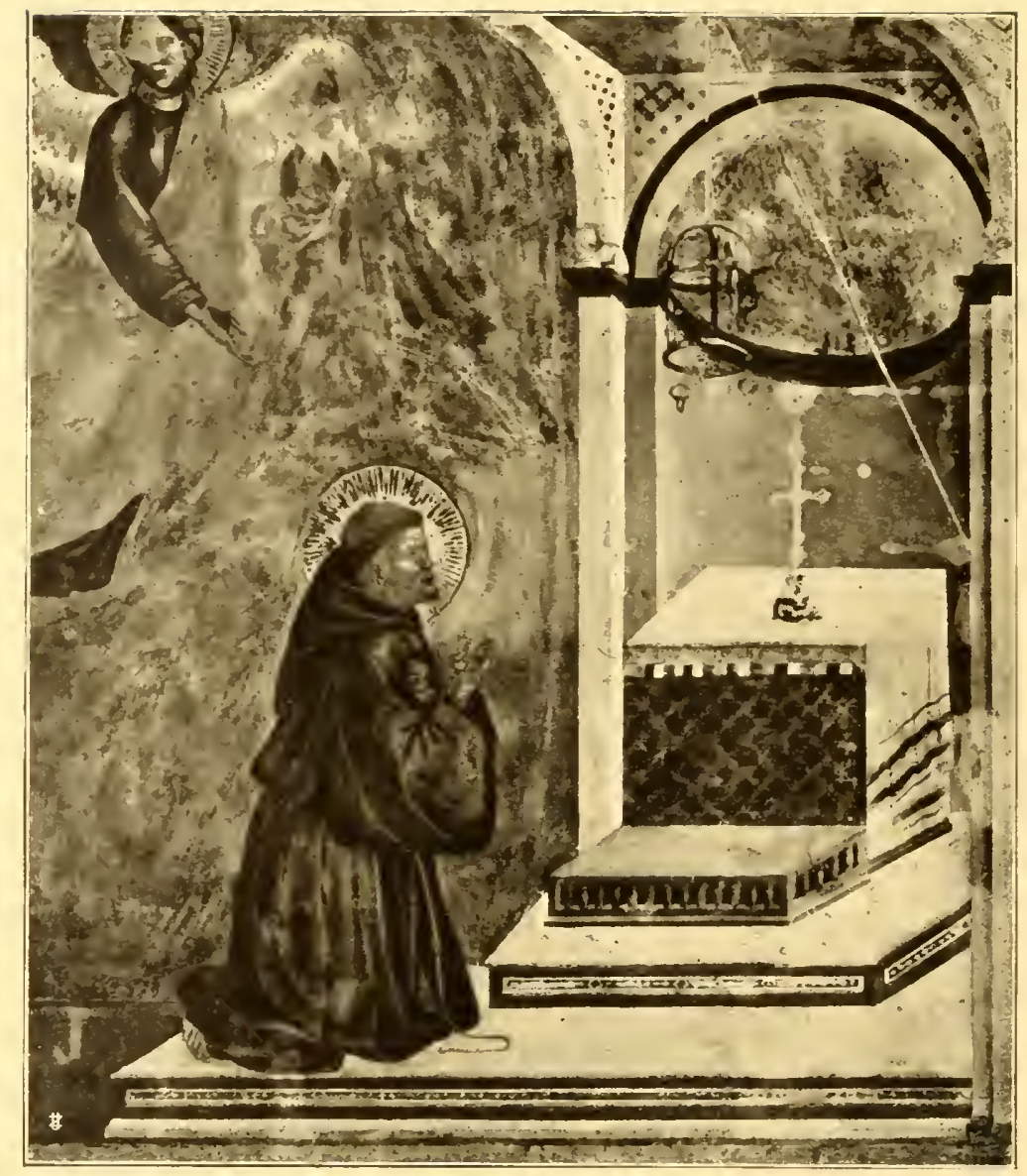

Fig. 2. GIOTTO: IFFRESCO IX S. FRAYCESCO D'ASSISI,

liane a caratteri gotici in leggiarlri cartigli.

Tondimeno eotesti innovati drappi. conservarono ancora un po" d'aroma orientale. fin quando. all'albeggiare del trecento. larte del telaio ahbandonando la Lucehesia. per cedere il primato a Firenze. Bologna e Venezia (forse per incentivo degli esuli. dopo la presa di Lucea). non si avviava nei nuovi centri ad affermarsi prettamente italiana.

La piì rapida e profonda trasformazione si verifico in Firenze sotto l'influsso della naseente civilà nmanistica sollecita a banlire le vechie forme convenute, ormai stanche di vagare. Ivi le prime stoffe. immemori dellinginnzione del Corano. accolsero ben presto nel campo de' propri ornati anche la figura umana. usandola specialmente ad illustrare di saere scene gli abiti liturgici.

Da questo punto e a questo officio. l"iconografia tessile con dignitosa libertà si attiene alla pittura quasi dagli inizii, prendendone il riflesso nel suo svolgimento. fino a' primordii del cinquecento: anzi, quantunque non se ne abbia notizia certa, si presmme collaborassero dirctamente a codesti fregi, artisti di merito e di fama, già 


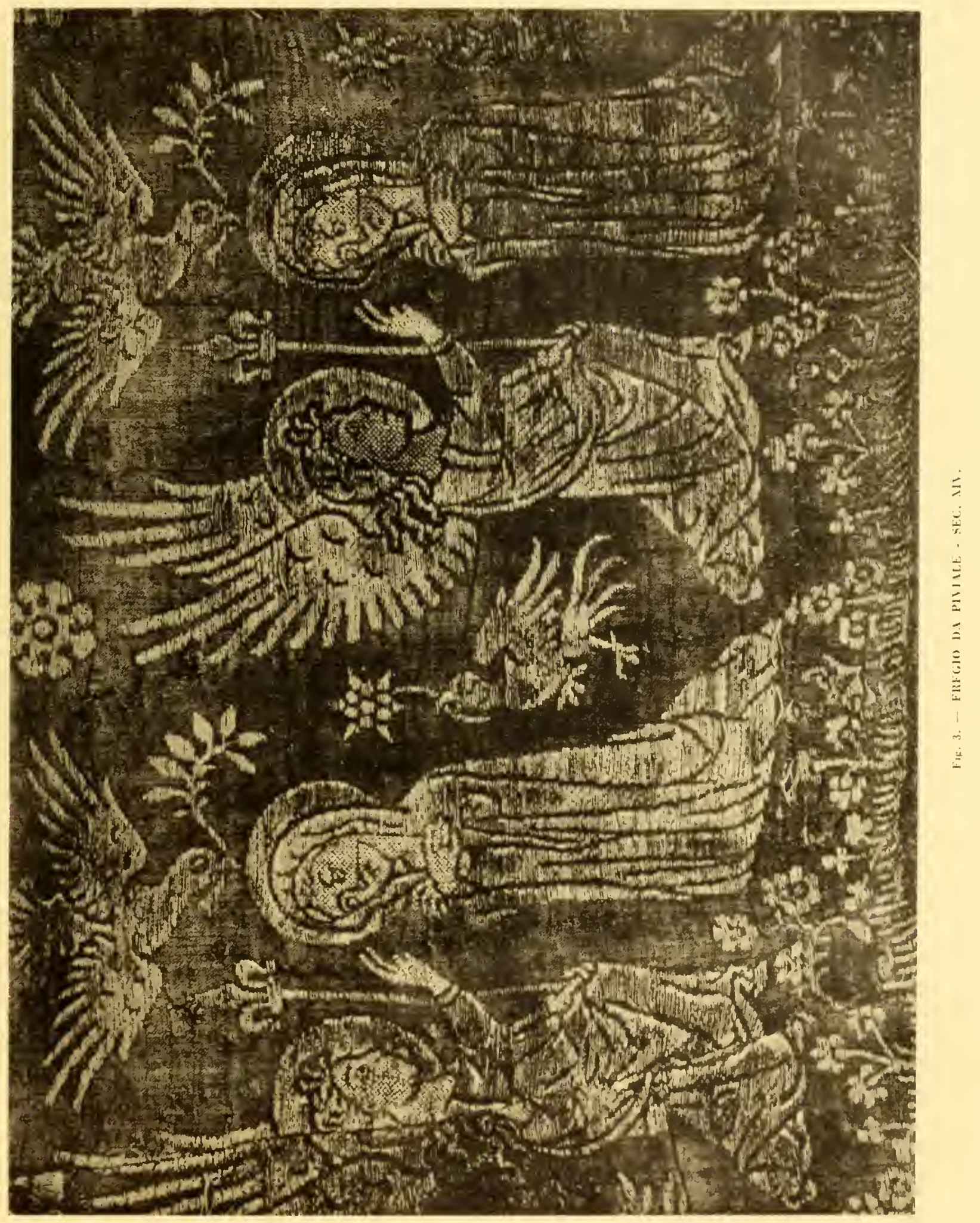




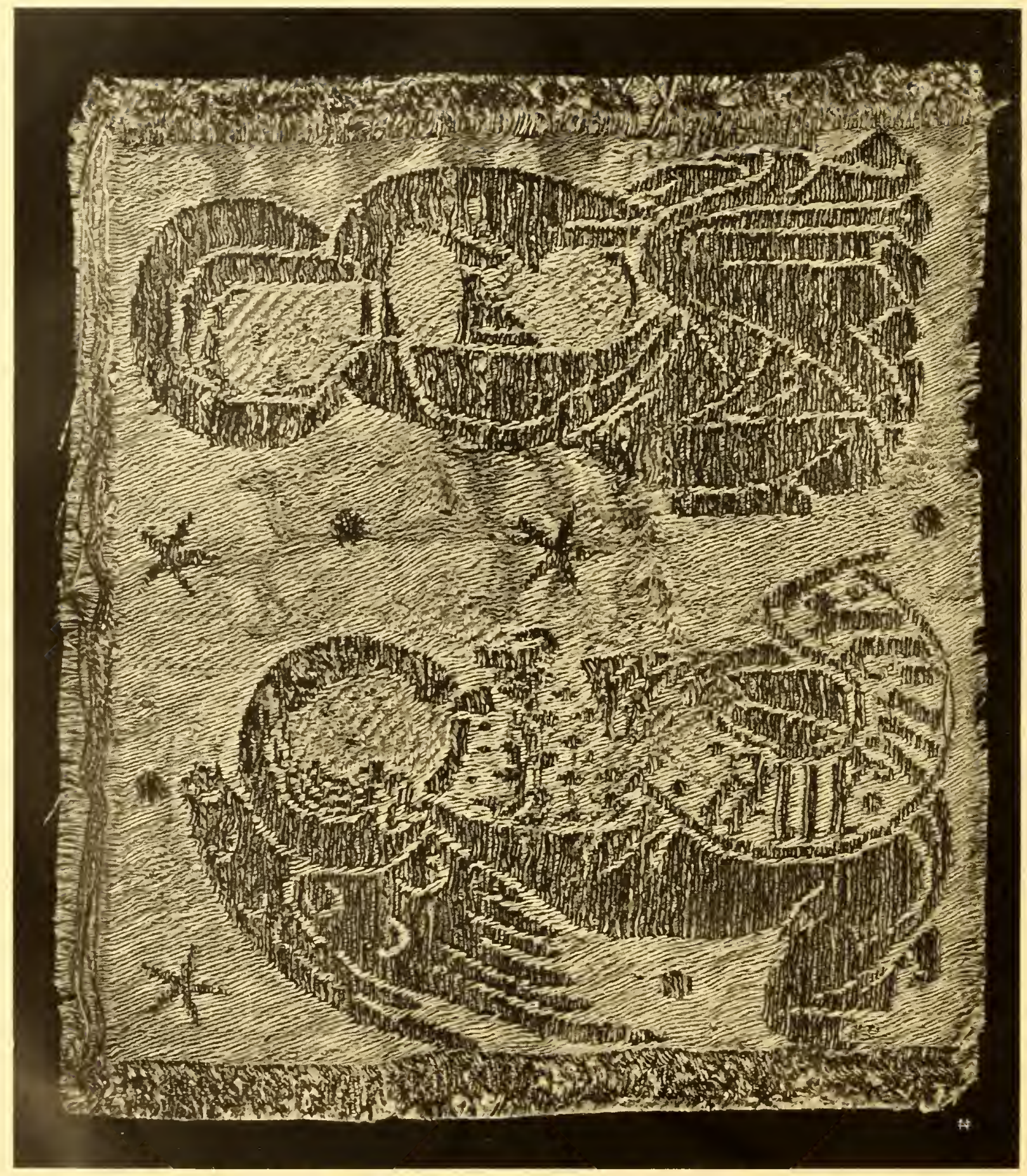




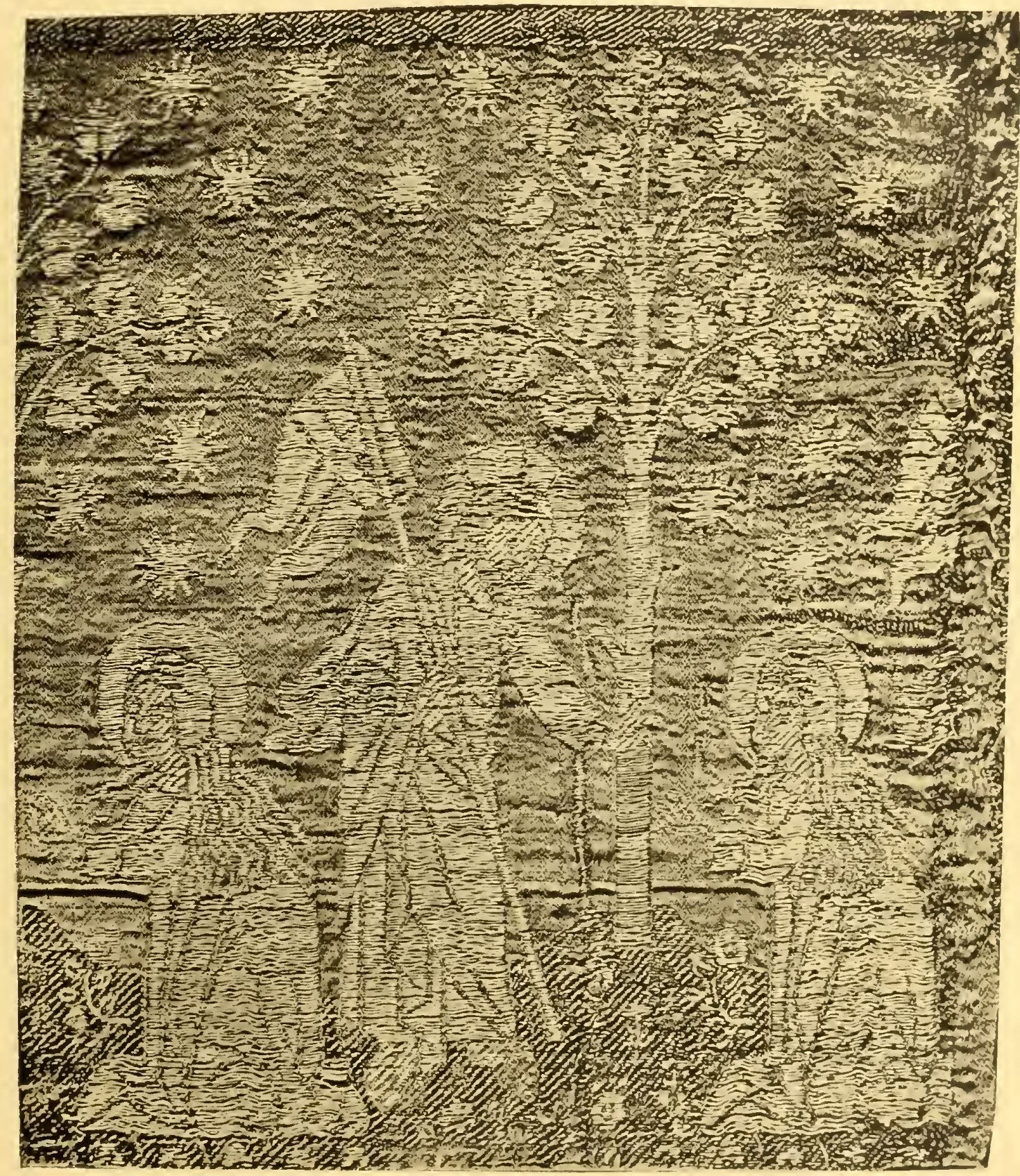

Fig. 3. - TEESLTO LITLRGICO DIL, SFC, XIY.

noti per la loro partecipazione allindustria del ricamo, quale più obbediente al vero pittorico ed anche più ricea e ricercata.

Fra questi Raffaellin del Garloo «si die. de - secondo riferisce il Vasari — a far disegni a chiaro-senro e fregiature di santi e storie per le monache ed altre genti rlie allora ricamavamo assai paramenti n; e dalla stessa fonte apprendiano dhe Antonio del Pollajuolo disegnava per la chiesal di 


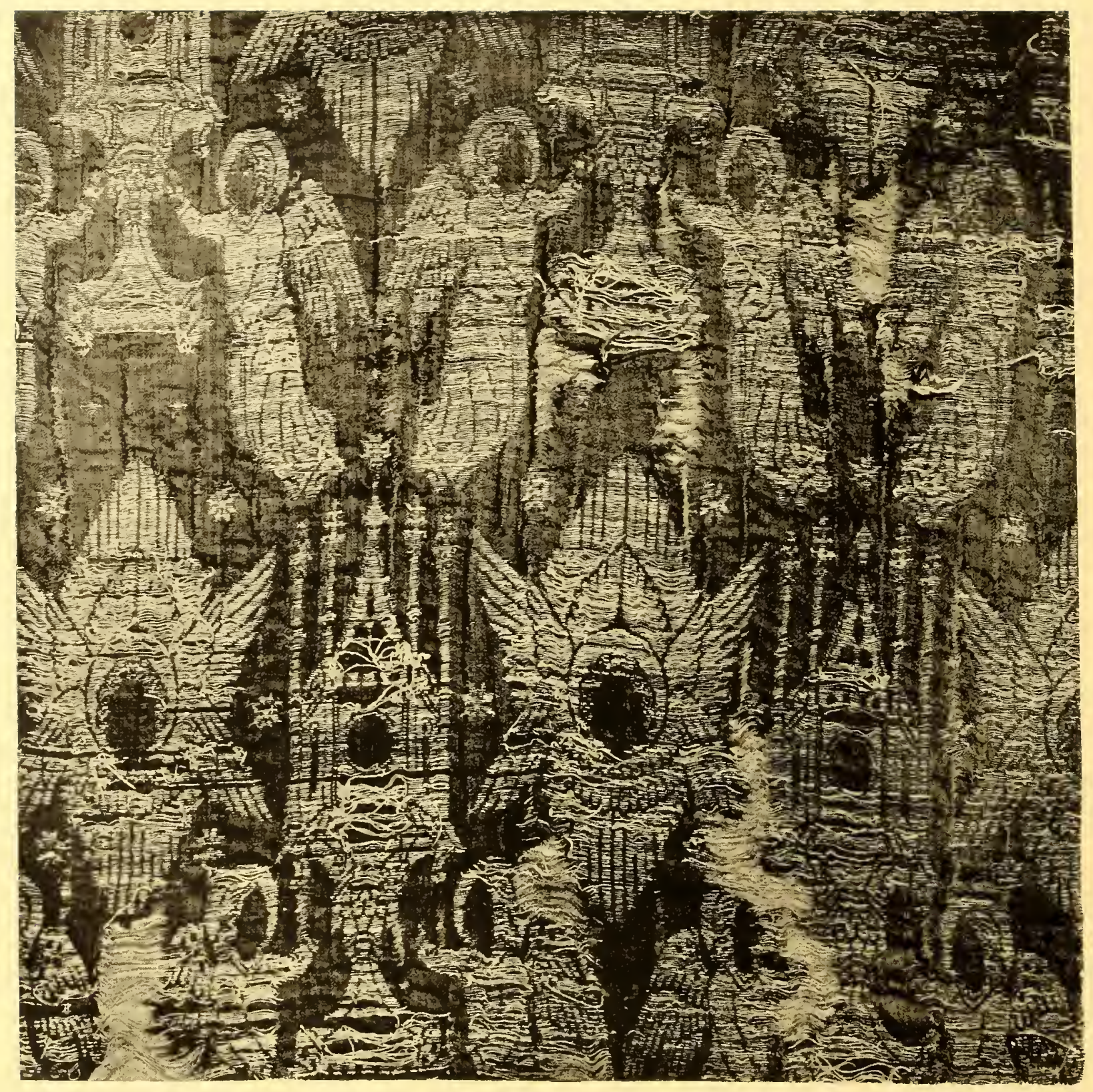

Fig. 6. - TESSUto LITURGico. FINE DEL SEC. XIV.

S. Giovanni due tonacelle e $u$ piviale. $i$ cui fregi sono nel Museo dell Opera di Santa Maria del Fiore. Si attribuiscono invece a Justus di Gand i ricami del piviale engubino; a Luca Signorelli le dalmatiche nella cattedrale di Orvieto (Venturi); e a Botticelli il cappuccio del Museo PoldiPezzoli in Milano.

Alla traduzione dei disegui di questi ed altri artisti non mancarono fedelissimi ricamatori. famosi pur essi nellarte loro: Paolo e Piero da Verona, Giovanui da Ma- 


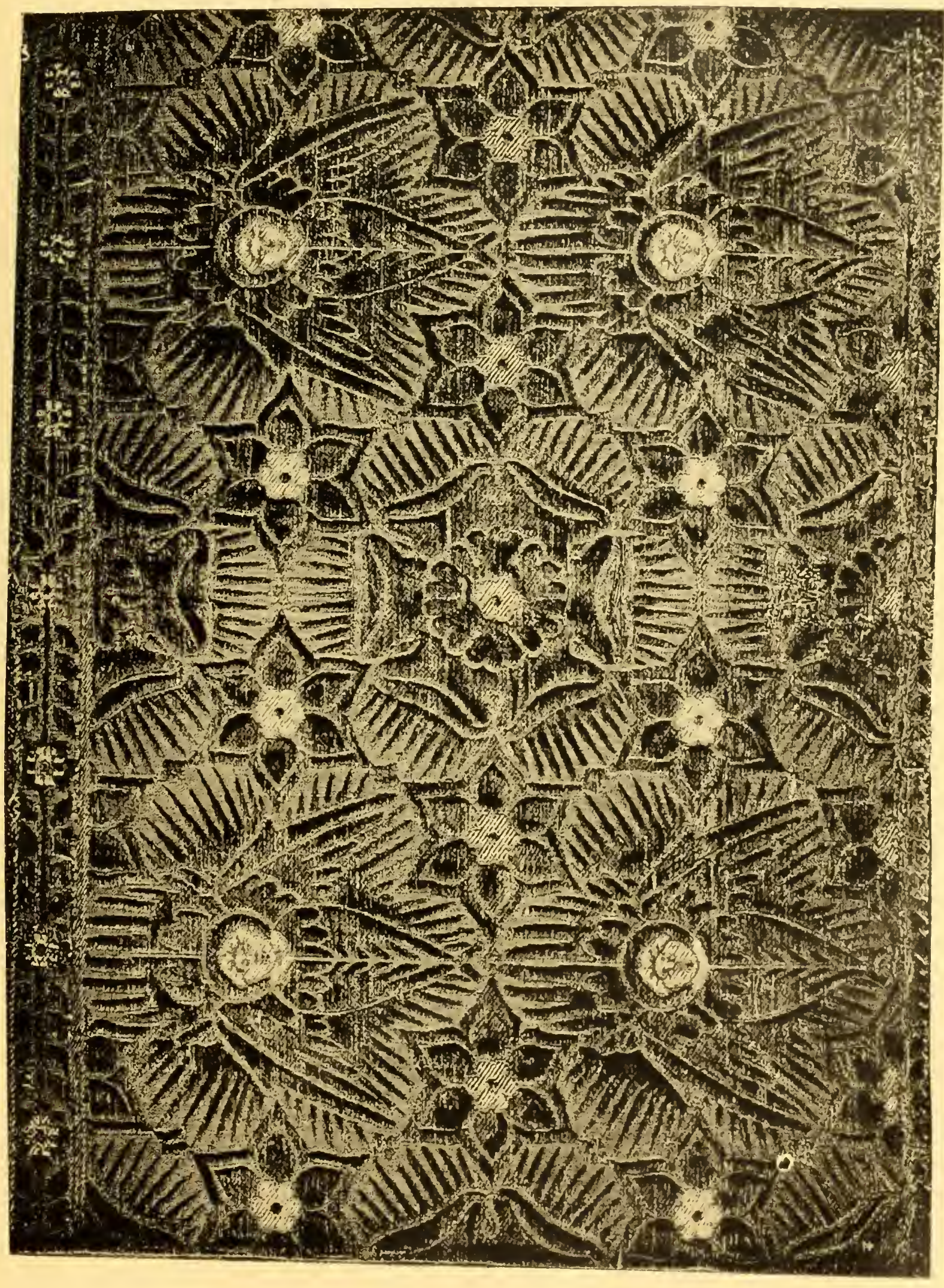




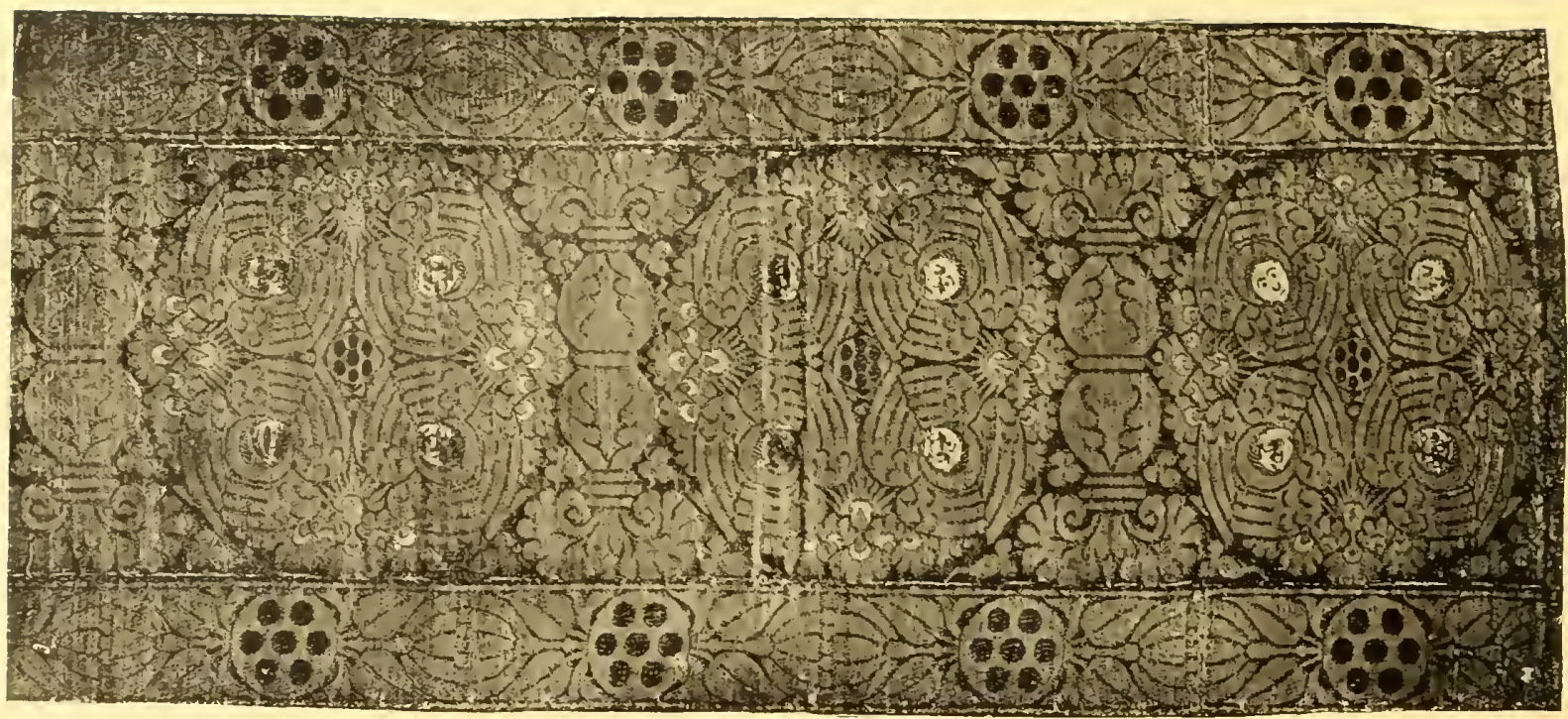

Fig. 8. - FREGIO II DICEO DI PIIIALE.

lines, Jacopo francese e compagni. interpreti del Pollajuolo; Nicola V'eneziano. lodato nella vita di Pierin del Vaga raro ed unico maestro di ricami; e poi Giovanni Battista, Gerolamo da Firenze, Federico Tedesco e molti ancora, al scrvizio d' Innocenzo Ottavo. A quanto si vede il ricamo non era allora virtù femminile!

Sebbene in altro luogo avenmo occasione di osservare che lindustria tessile ha un suo svolgimento autonomo dal ricamo. in codesto speciale gruppo figurato l'ha invece parallelo, ariegrgiando talvolta, con tecnica fiù corrente e meno dispendiosa, le acu. pitture dei sacri paramenti.

Pochi cenni tecuici. e passiamo in rasscgna. per ordine di tempo, qualche tipico esemplare di codesti fregi. mettendoli a confronto, laddove non è possibile trovarne la paternità. con le singole opere che si presume dettcro l'ispirazione più o meno diretta all'anonimo disegnator drapporum.

I più antichi fra di essi, risalgono al se- colo a cavalicre fra il tre e il quattrocento, e si disinguono subito oure che allo stile. per la tecnica che è quella vecchia di Lucca. dovuta al telaio Jetto di Giovanni il Calabrese, c riconoscibile per le caratteristiche cimose formate da due o tre cordicelle parallele che reggono l'orditura in seta spolinata d'oro membranaceo.

La fonte principale dei soggetti che liil. lustra è il Nuovo Testamento anzichè il Vecchio. a cui attinse più spesso l"alto medioevo: ed ogni scena relativa è incorniciata sui teli in tanti rettangoli disposti a scacchiera, per dividersi in file orizzontali o verticali secondo siano da riportare su pianete o su piviali.

La figura 1 riproduce uno dei più antichi quadretti del genere rappresentante Santa Maria Egiziaca genuflessa innanzi all'altare, tutta coperta le chiome; mentre in alto pende la mano divina, e da mollbero sta per spiegare il volo un aquila di tipo araldico. Il fondo n’ è azzurro; ed il soggetto, 


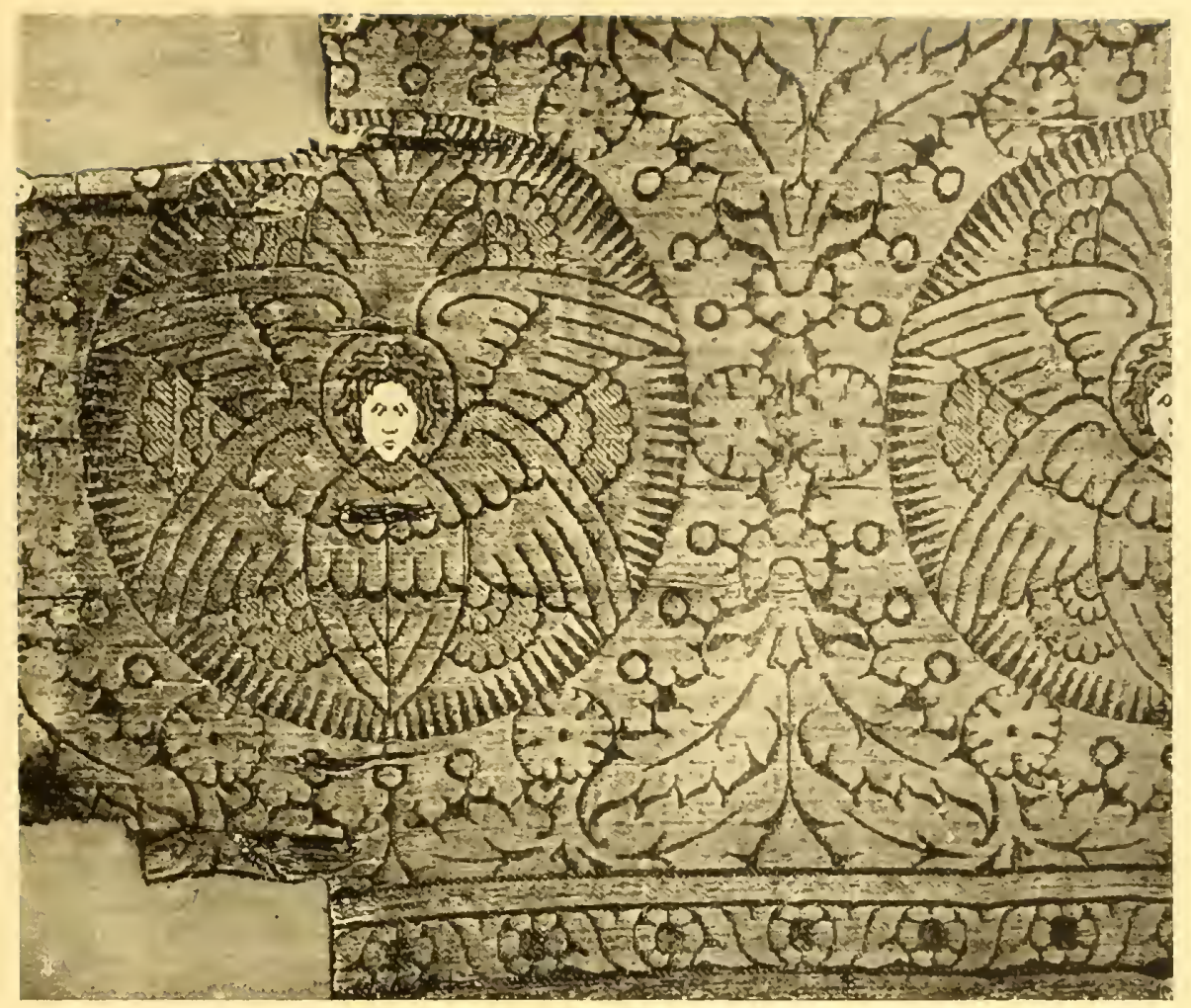

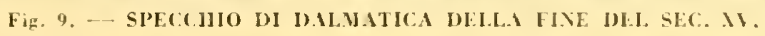

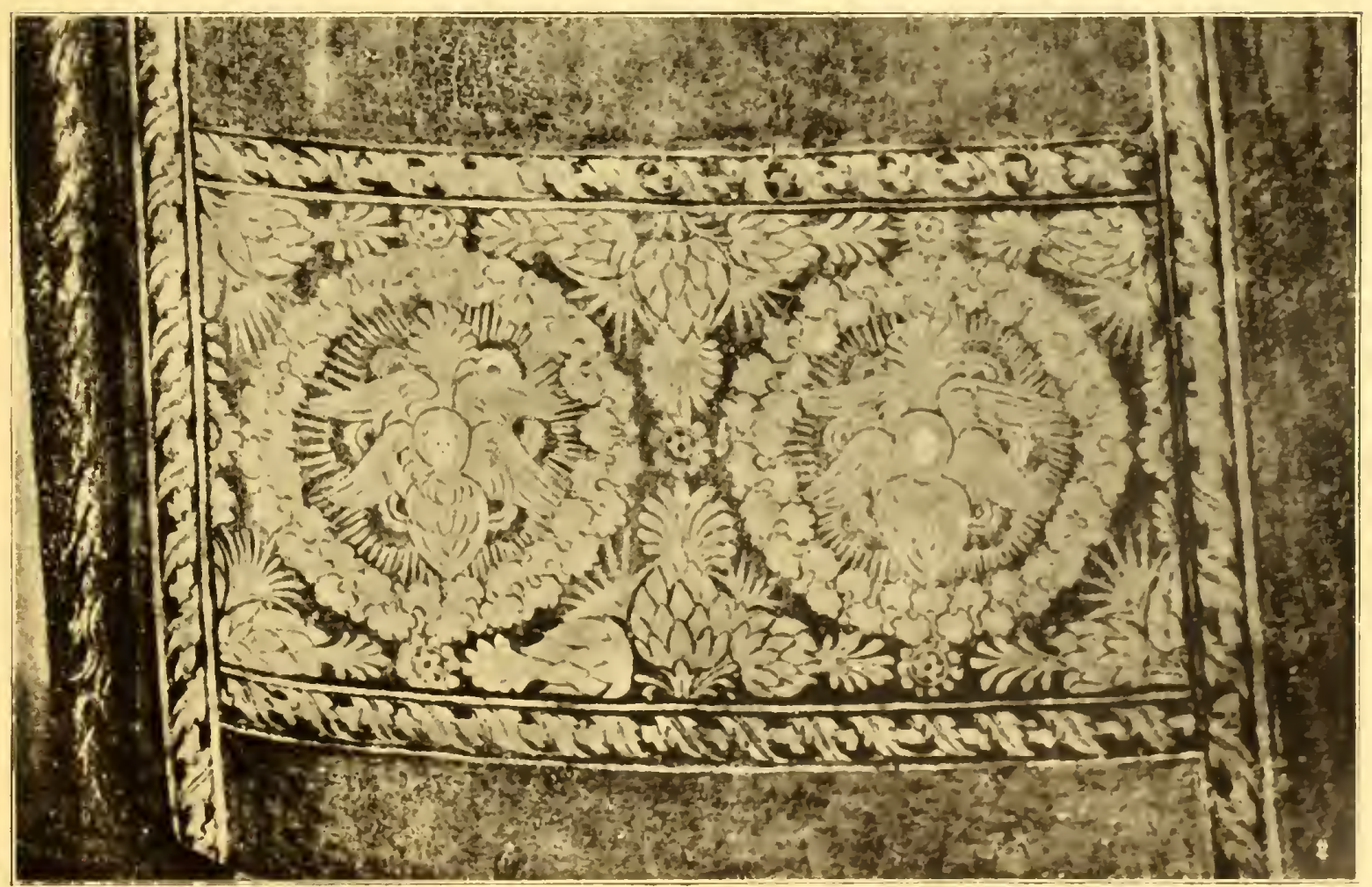

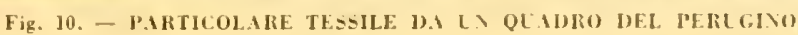




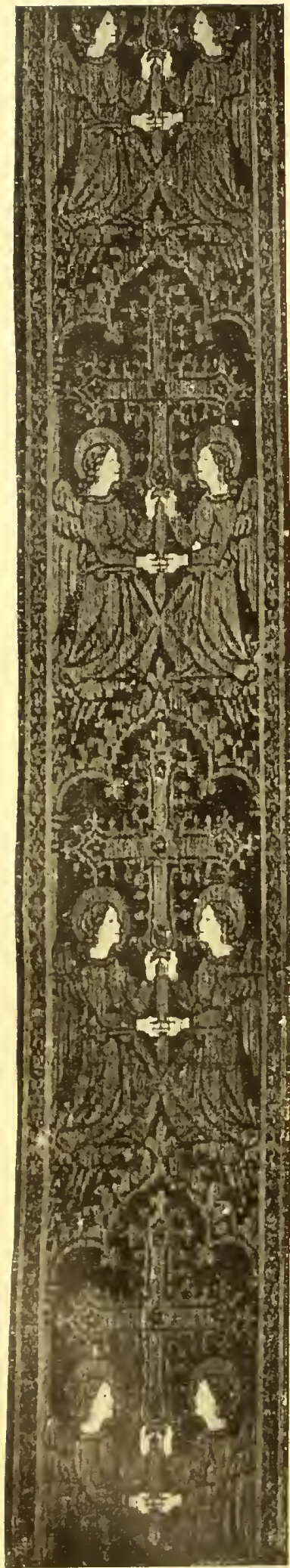

in oro con alcm poco di candido che lo illumina. si pui porre a confronto stilistico e formale con la nona Storia di San Francesco, affrescala da Giotto nella cappella superiore della Cattedrale d'Assisi (fig. 2). Ivi l'altare nella sua prospettiva a rovescio, con sopravi la croce equilatere in prospelto, e la figura posta da un lato come per lasciar patente il palio e la pre. della, sono ingenuità di composizione clıe si rispechiano fedelmente nel tessuto. c in esso più evidenti per l'assenza del chiaro-scuro pittorico.

A questo primitivo saggio ne segue a lireve distanza di tempo un altro con la scena dell' Anmmeiazione replicata in serie $o$ rizzontale (fig. 3). L'Angelo di fronte alla Vergine, tiéne uno scettro gigliato alla maniera angioina; e il fondo ì animato di grandi uceelli esotici dallaspetto rapace che non voglion ancora cedere il campo alla mite colomba simbolica.

Ma liberi ormai d"ogni ricordo atavico sono gli esemplari segnenti. Ecco lo stesso soggetto di sopra con la Maria assisa sovra uno sgabello. che neghittosa serra le mani al seno come fanno le vergini di Simone Martini innanzi al Messo. ivi recante invece del giglio, un polizzino che dice AVE MAR [IA]. Alle figure vestite d'oro $\mathrm{i}$ visi e le mani sono dincarnato, e il cielo con poche stelle. roseo intorno (fig. 4).

Altrettanta delicateza d'ingenno sentimento spira dalla scena del Noli me tangere, espressa nel broccato fig. 5; dove si vede Cristo e la Maddalena in replicata effigrie sullo sfondo del giardino fiorito della Resurrezione, sotto un cielo stellato come un blasone.

In altro cielo di purpureo raso si elevano coppie di angeli. recanti ostensorii fra scrafini maggiori ( $f$ g. 6 ); e alternati a rose raggianti. gli stessi serafini. si delineano in uII drappo di seta verde ( fig. 7); e raggruppati a quattro fra nubi increspate, e formanti insieme mote alterne a balanstri, li ritroviamo nel fregio figura 8 , con ai margini stemmi medicei a sette palle rosse.

Lo specehio di dalmatiea a singoli chernbini fig. 9, sebben simile al precedente. e pur degno di nota. perchè, salvo lieve varianza, si ritrova nei colori originali, inclosso al S. Lorenzo allato della Vergine nella pala vaticana. dipinta dal Perugino nel 496 ( fig. 10). Qui l'identità fra tessuto e dipinto, riesce doppiamente interessante nelloffrir(i 11 termine rronologico preciso. estensi. bile allintero gruppo di codesti fregi serafici. e nel confermarei quanto fedeli fossero gli antichi maestri nel ritrarre il vero 


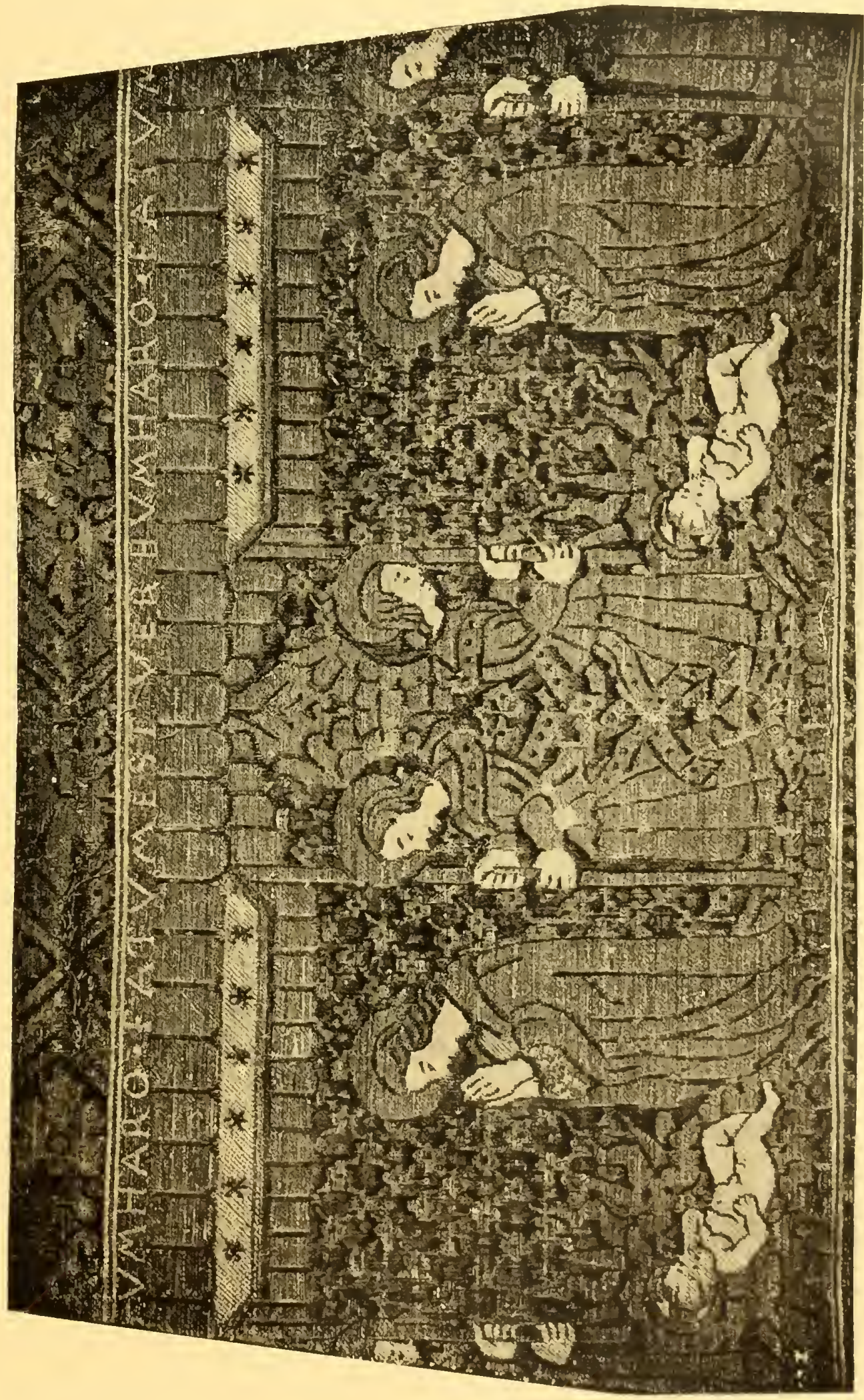




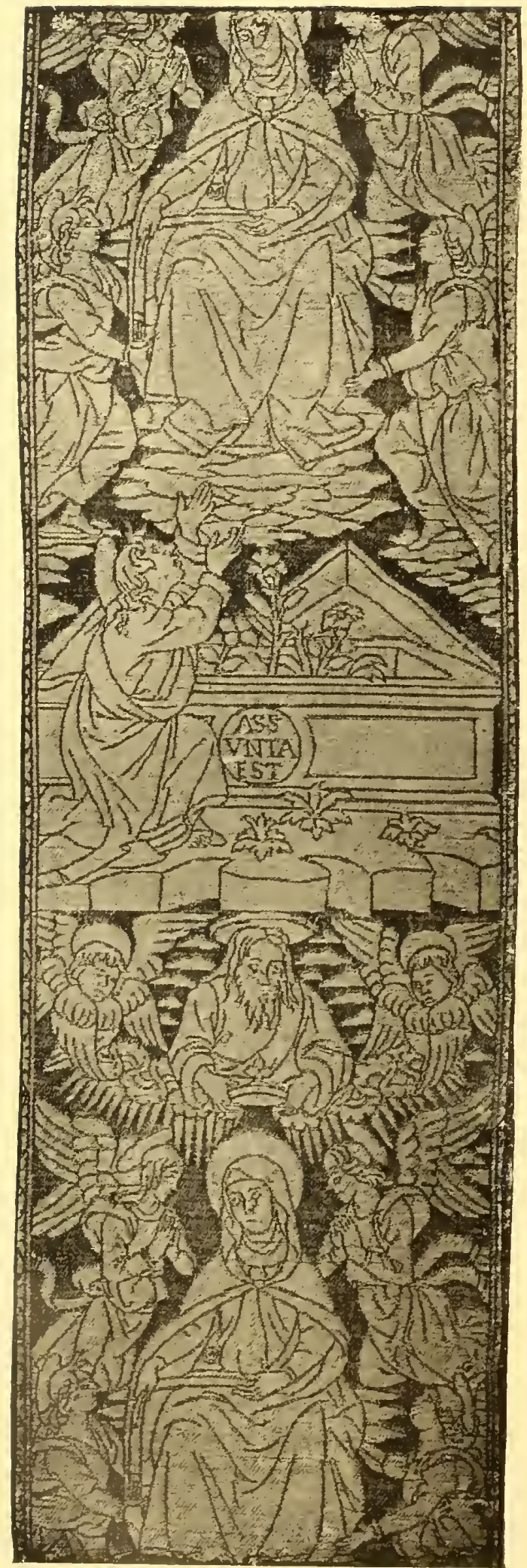

Fig. 13. STOLONE ATTRIBUITO AL GIIRLANDAIO. anche negli accessorii.

Lo stile sobrio dei saggi fin ora ammirati, con le loro figure poste tutte in un piano, senza sfondi architettonici, nè prospettiva d'ambiente. come si addice al huon gusto tessile, che sa opportunamente rimunziare alla terza dimensione, comincia a divenire ivi innanzi manierato e pittorico; e al de. cadimento artistico si accompagna quello tecnico, dovuto alle condizioni dellarte di Por Santa Maria, poco floride per la cre. scente concorrenza dei nuovi centri dell'Ttalia nordica, e forse in parte a più severe restrizioni suntuarie. Onde l'oro vero viene surrogato con il falso. e la seta col filugello, trasformando broccati in broccatelli; mentre speriali telai, larghi quanto i fregi da farsi, battono le nuove materie simulatrici in lunghe striscie ad altoliccio.

Lo stolone con angeli crucigeri entro nicchie trilobate fig. 11, già denota tale scadimento dallorpello rado e intessuto in economia nel senso dellordito; cosi pure questaltro fregio fig. 12 , con la Natività intitolata Verbum.haro (sic). Fatum (sic). Est.. ha gli stessi difetti intrinseci; nondimeno lo stile in entrambi conserva dellantica sobrietà silografica.

Ma in sul principio del cinquecento i rapporti fra la pittura e l"industria dei nostri fregi vanno stringendosi: $i$ disegni loro rivelansi più liberi c tanto più prossimi ai cartoni originali da suggerine talvolta l'autore, come gli arazzi famosi.

Nel fregio fig. 13 la raffigurazione della Vergine assunta in gloria su l"arca fiorita, dall'epigrafe Assunta Est, ricorda molto davvicino la maniera del Ghirlaudaio; il quale, avendo idleato disegni musivi e ricani, non è improbabile tracciasse anche 


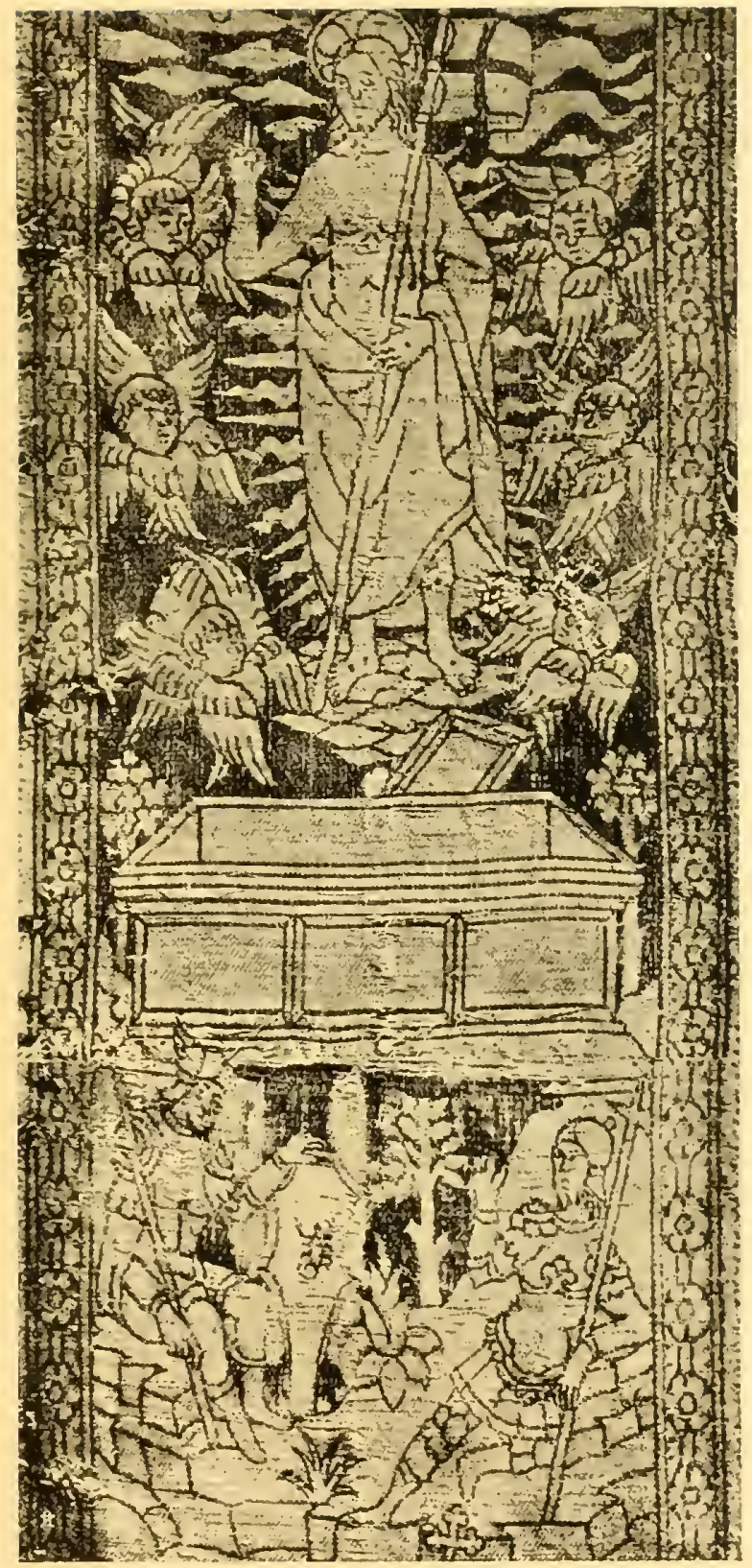

Fig. 14. - STOLONE ATtRIBLito A RAFFAELlix DEL GaRBo

modelli da tessere.

Egualmente a Raffaellin del Garbo rieamografo. si possono attribuire non poehi di codesti tessuti istoriati, come per esempio to stolone fig. 14, the si avvicina alla di lni maniera nella Resurrezione di Cristo, dipin-

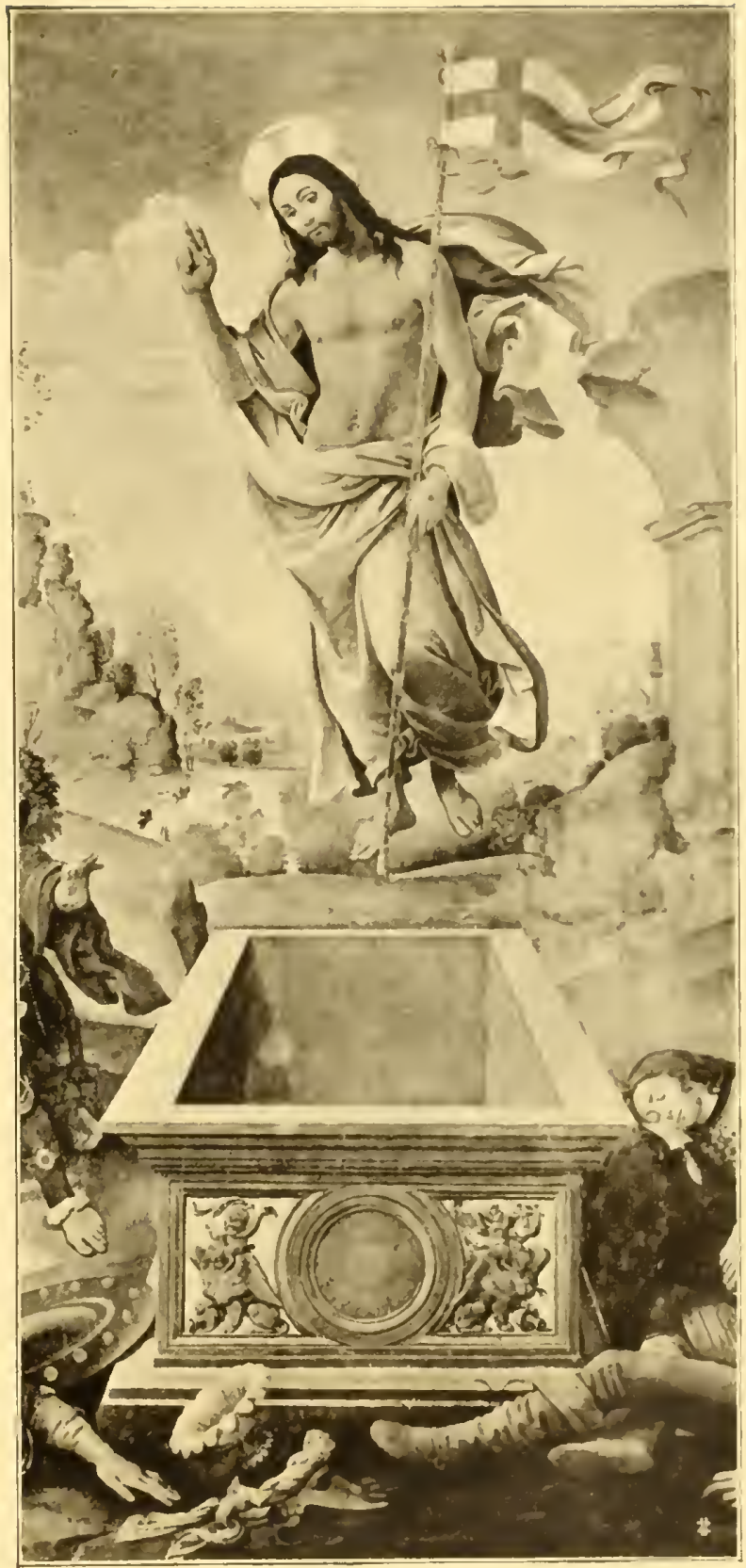

Fig. 15. - RAFFAELLI. DEL GARBO: RESLRREZIOJE.

ta per la famiglia Capponi (fog. 1.j).

Se non del Verrocehio stesso. certamente dedotto dal gruppo dell'Incredulitio di S. Tomaso (fig. 17). i il disegno del fregio $f i$ gura 16, ove anche la nicehia architettonica è resa con intenzione prospettica. 


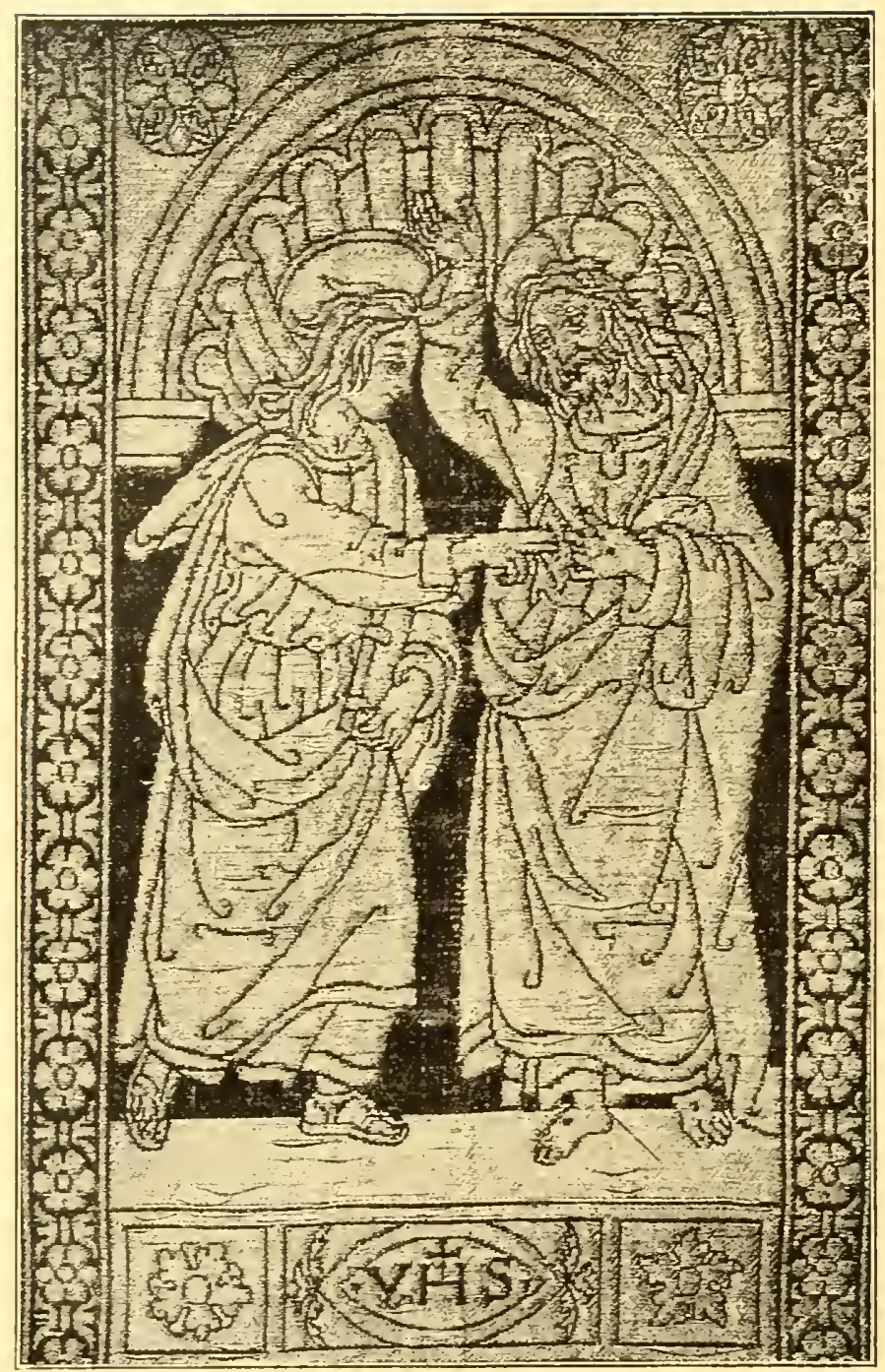

Tig. 16. - STOIONE ISPIRATO AL IERROCCHIO

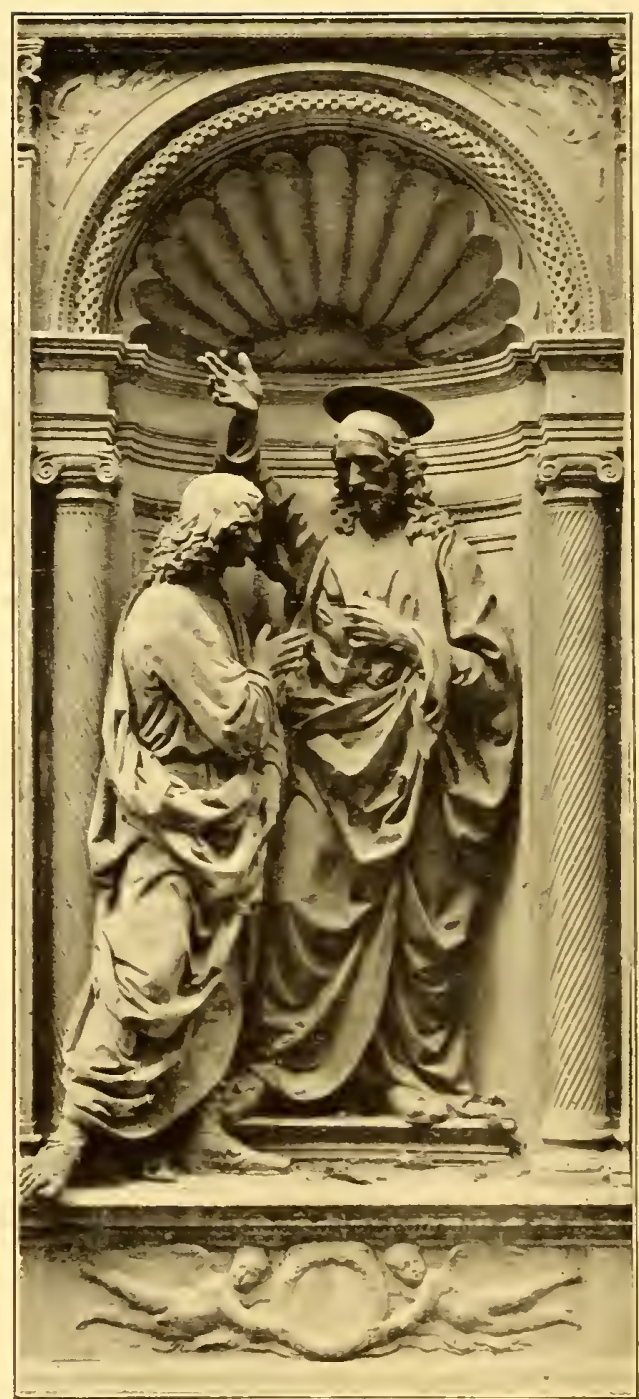

Fig. 1:- IERROCCHO: IVCREDLITA DI \& TOM.ASO
Chindiamo questa breve rassegna con il cappuccio piviale fig. 18. di cui uno simile descrive un inventario del Duomo di Orvieto. in data 1560: "cappuceio di broccato riccio con una pietà ). Aggimngasi che il broccato riccio nel nostro esemplare è in argento e oro. e mostra intorno al Cristo rami scerpati avvolti di un nastro col breve Venite ad me.

Anche siffatti cappucci tessevansi in con- tinuità verticale e si usava ritagliarli dalla pezza per riportare su piviali. che spesso avevano la testata nella stessa stoffa diversamente affigurata: per cui si può supporre che quello di Orvieto sia uscito dallo stesso telaio del nostro; al quale. per ragion tecniea. devonsi pure attribuire certi drappi in Santa Croce di Fïrenze.

Come si è visto non sono dunque questultime le edizioni principe dellarte tes- 


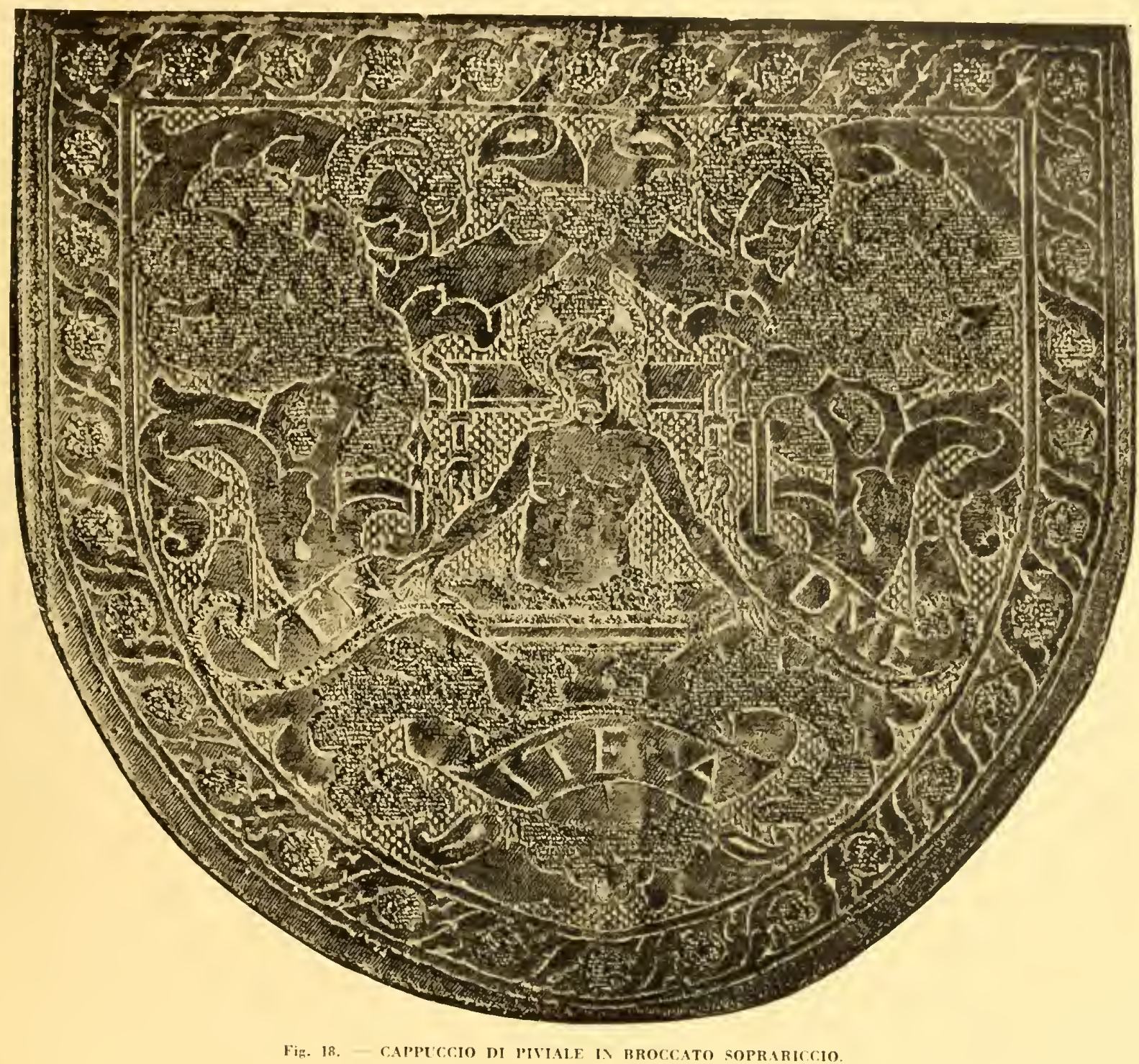

sile forentina, ben più famosa per $i$ suoi velluti e ricami, si hene semplici prodotti dozzinali espressi da torchi rotativi, quali sembrano codesti copiosi telai, mossi a di- vulgare modeste o pretenziose vignette sacre. che dalloro allorpello traducono arte aulica in vernacolo. 


\section{CONSIDERAZIONI SOPRA ALCUNI MO'TIVI \\ E SIMBOLI TESSILI}




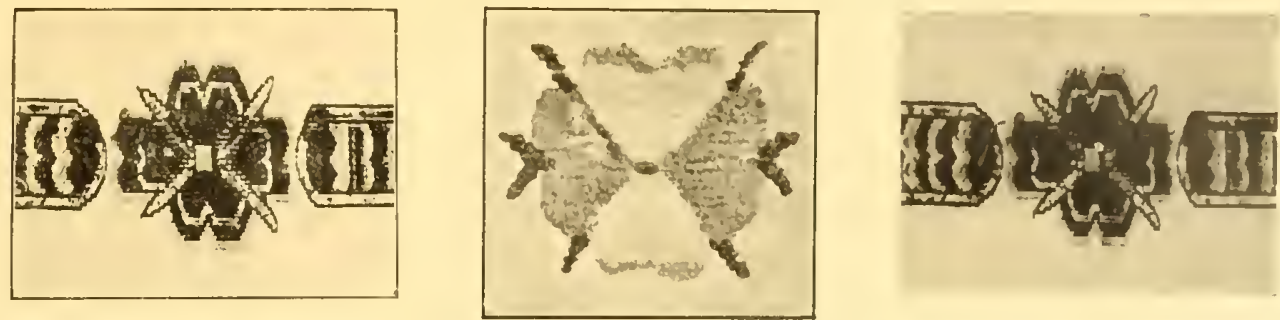

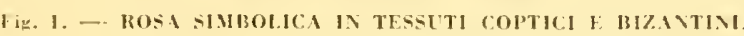

\section{CONSIDERAZIONI SOPRA ALCUNI MOTIVI E SIMBOLI TESSILI (*)}

Quale un'espressione del gusto corrente niun caupo sperimentale dell'arte si offre piì fecondo di considerazioni quanto quello tessile. In esso si può rintracciare nel corso de" secoli lorigine e lo svolgimento di certe forme selezionate; il divenire e l'affermarsi degli stili: il rangiare della sensiluilità coloristipal e della perezione della forma: il trasmigrare dei simboli; e. in mua parola. vi si possono seguir le leggi centrali delli vasta sfera dell'arte nei loro svariati fenomeni.

Dove meglio else non ivi la fortuna di ecrti motivi rivela più chiaramente il modio gusto collettivo. dovuto a quellorgoglio di superamento individualistico. che nel limite del prudente misoneismo. ronduce nondimeno per gradi al grottesco inavvertito della moda?

Dopo quella trecentesa. ad esempio. delle stoffe animate dogni specie zoologiea. venuta dall'Oriente, col maggior sviluppo del. lindustria nazionale. subentro l’altra dei motivi vegetali, più agevoli a disegnarsi $e$

(*) Da L'Arte, ammala XXVII, fasc. II-III. vicinanti meglio il gusto nostro: cosicchè in sugli ultimi del secolo decimopuarto e $\mathrm{i}$ primi del quinto i consueli gruppi animali, posti in isole rocciose, si fanno sempre piu radi e rari; mentre invece spessegriano gli arbusti, e saccreseono in alloeri salienti, serpeggiando per tutta la lungliezza del telo che li accoglie, fino a costringere il disegnatore a semplificarne liutricata strutturil. tracciando eon un sol tratto i doppi profili contigui dei loro rami contorti e delle foglie aceartocciate l’una contro l'altra per la troppa esuberanza.

Onde poi, al primo Cincuecento, la voga dei velluti cesellati, che sono appunto gli ultimi di rodesto tipo giunto a maturazione e già i primi di uno novello: c così via altri rezzi estetici si avicenderanno, come con egual passo, nelle arti figurative, allo stile attivo segue il manierismo passivo. fino all'emancipazione.

Per il colore avviene altrettanto: nei lnoghi ore si predilige la forma. le tinte delle vesti sono 111 po agrette e impertinenti. ma i motivi condotti con meditato garbo. Così in Firenze per le stoffe come per la pittura: 

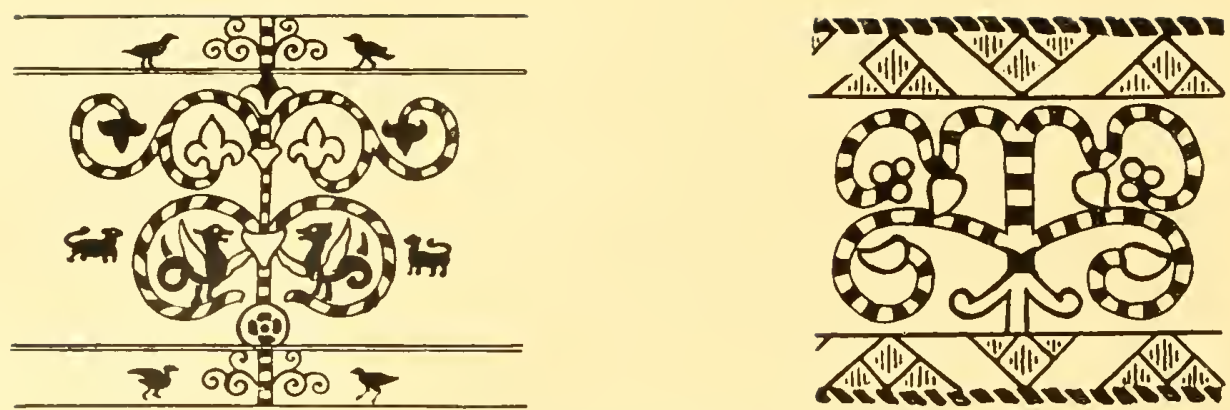

Fig. 2. - VOTIVI SIMLLANTI LALGA "CALLYTHOMNILW" IN TESSITI PAXORMITANI.

mentre. di converso. in Venezia il colore vive nei suoi più forti contrasti in tutto il Quattrocento, finchè, esaurito. dileguasi nel dolce chiaro scuro, cui rispondono i tardivi profondi velluti alto-bassi e $i$ cangianti damaschi tono su tono.

Ma non la sola ragione estetica governa il formarsi e il cristallizzarsi dei motivi: come la religione e la storia sono fonti alle arti plastiche. alla tessile i simboli. Quando per secoli, al variare dellambiente estetico vediamo resistere tenacemente un dato motivo. siamo indotti a pensare che in esso si riponga un significato più stabile della moda volubile.

Esemplifichiamo. Negli arazzi coptici. tra gli altri tanti emblemi intessuti. notasi specialmente una corolla a quattro petali cuoriformi e sepali altrettanti; la quale ritorna ancora più diffusa nelle porpore alessandrine e nelle bizantine, sia nella precisa forma indicata ( $f$ g. 1 ), sia scomposta in foglie, talvolta sovrapposte a formare grossi 3 in fila, intorno alle consuete scene venatorie di fonte sassanide.

La diffusione stereotipa di codesto ornatino non poteva sfuggire a quanti prima di noi studiò stoffe medioevali. Il Dalton (1) unitamente allo Strzygowski (2) lo deriva- no dalla sovrapposizione delle due foglio. line cuspidali della palmetta composita, credendo riconoscerne l'archetipo in certi ornati architettonici della Grecia. da cui sarebbe passato. per il tramite della Persia, all"Egitto. Il Falke (3), invece. lo identifica col fior di loto. Ma alle due ipotesi mancano quegli anelli intermedii necessari a stabilire continuità di parentela fra tipi simili nella prima apparenza.

A noi sembra più verosimilmente riconoscibile nella quadruplice corolla un semplice antemio della famiglia delle cruciferae, quale la Rosa di Gerico. l’anastatica; il cui significato simbolico dell'immortalità e della Resurrezione può solamente averle data tanta resistenza di vita in tempi cristiani, quando appunto le arti del disegno erano ideografiche, se non addirittura geroglifiche.

In quanto alla cronologia del simbolo, la presenza d'esso nella vera specie di fiore, qual fu trovato nelle mani di una Thäis di Antinoe, defunta nel IV secolo d. C.. ci consente, sulla scorta del Gayet, a riportarne il mistico significato avanti i tempi dei pellegrinaggi in Terra Santa, ossia ai primi dell'era cristiana (4).

Appoggia altresi la proposta identificazione, il valore simbolico di altri emblemi 


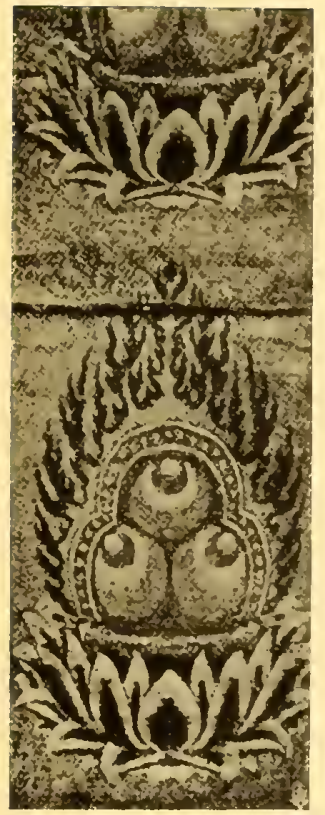

A
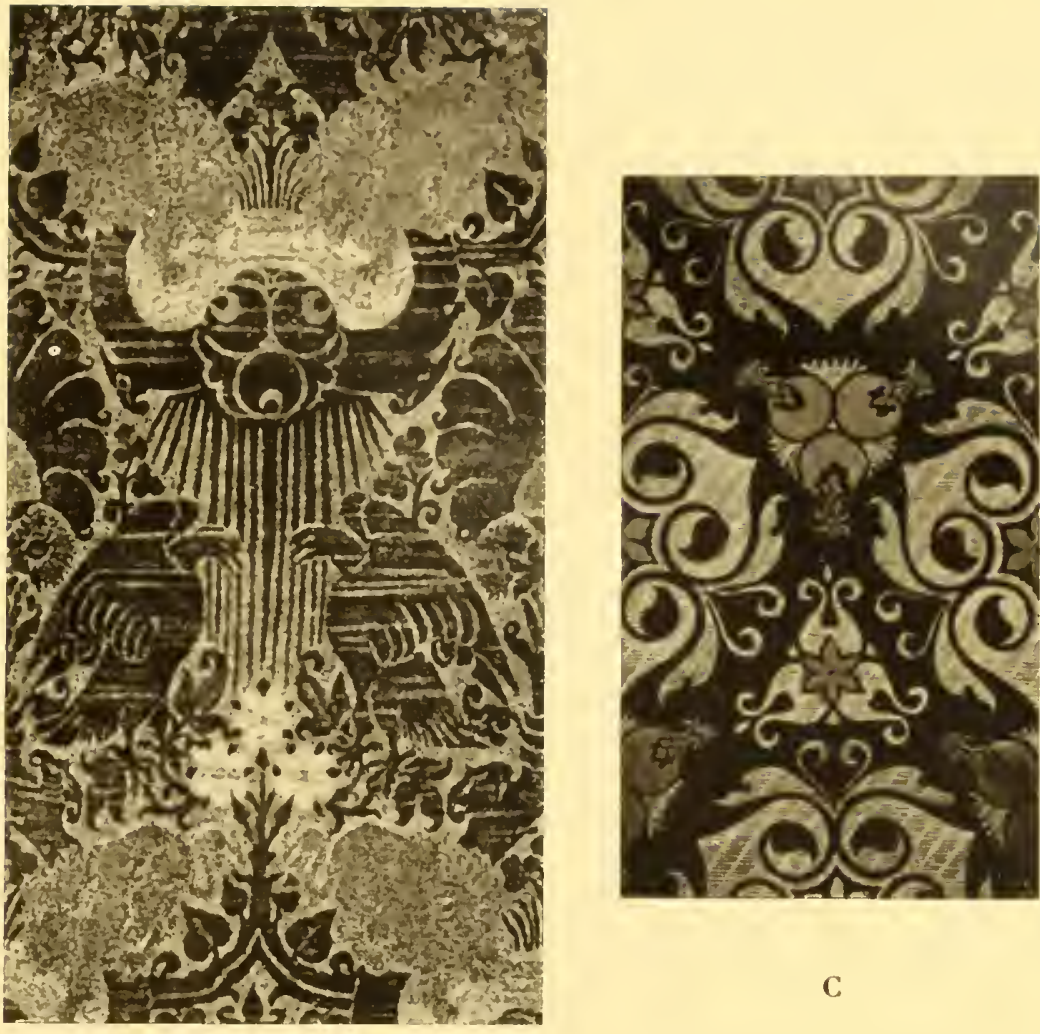

C

B

Fig. 3. - ALCLNE TRASFORMAZIONI DEL SIUBOI.0 CINTSE "LIEN"

che troviamo abbinati alla nostra rosa negli stessi tessuti coptici, come, tra gli ovvii, la croce e il paniere encaristico (5); nè può contrastarla la botanica considerazione dhe l'Anastatica hierochuntica sia mombrellifera, giacche appar chiaro. per l'esigenza pratica del disegno, che linflorescenza tipica a detta specie dovesse venir considerata come un insieme di singoli fori congiunti naturalmente in mazzuolo.

Un'altra pianticella peculiare a un gruppo tessile ritemuto panormitano del serolo XII, ̀̀ l’alga callythomnium (fig. 2). II gruppo che la reca in forma sintetica di cespo a segmenti rossi e oro, ì delimitato dall'uso speciale degli oggetti. che sono pas- samani di mitrie e pianete. o pure stole e cingoli. Fra i monumenti che meglio valgono ad illustrarlo vanno menzionati il cingolo di Geglicelmo Il di Sicilia. nel T'esoro Imperiale di Vienna (6): altro pertinente alla donazione di Eurico il Santo. in Ratislona (7); il passamano della mitria del Vesoovo Hartmann. nel Duomo di Bressanone (8): e altro ancora sulla casula di San Giovanni Angreloptes. nella Metropolitana di Ravemia (9).

In collesti passamani la pianticella stilizzata. ele n’ altrimenti si può individuare che nel callythomnium (tipico por le ramificazioni tubolari a segnenti rosis). (he il mare hutta in ablondanza alle rive mediterranee e par- 

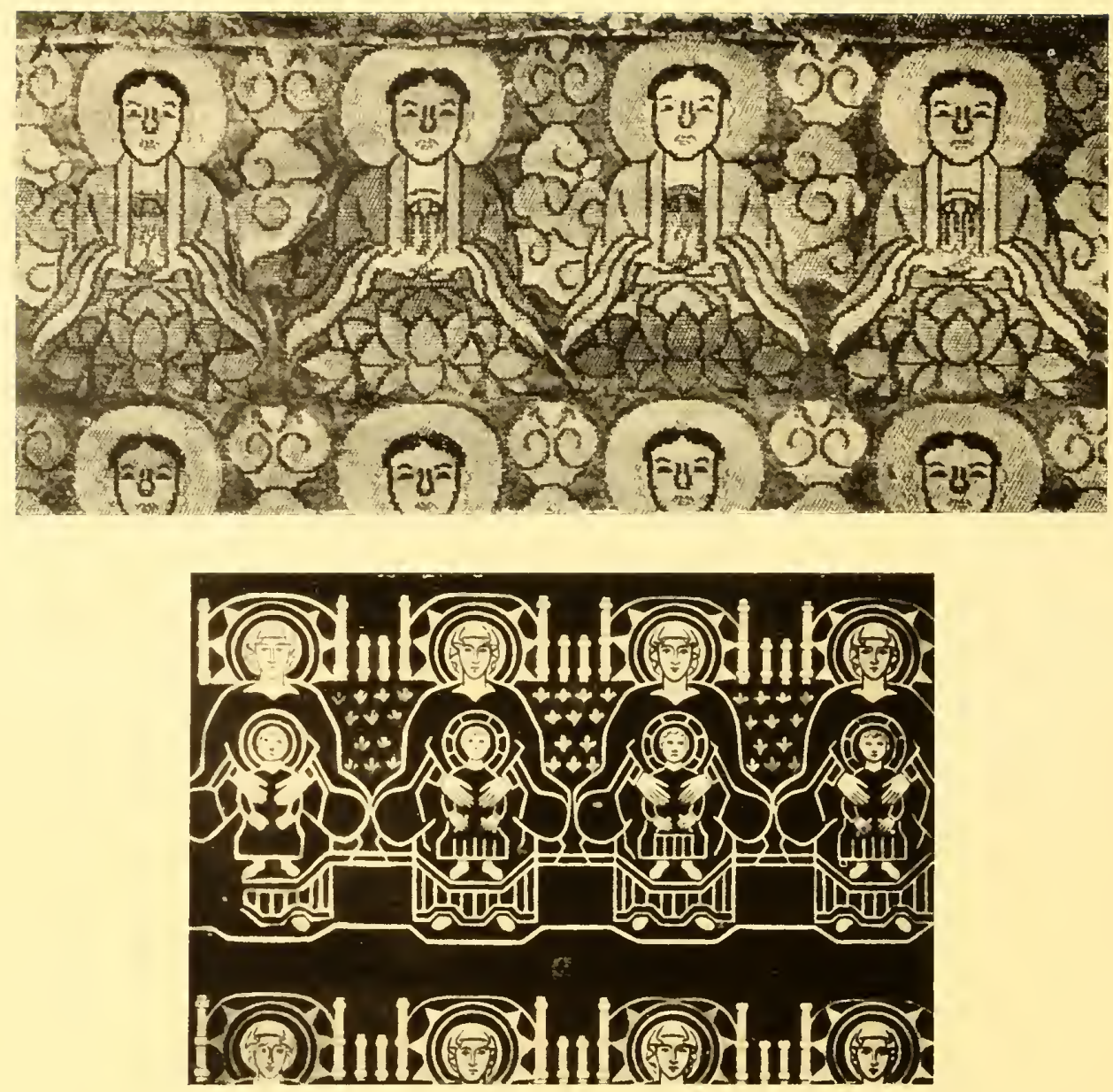

Fig, 1, - I "CENTO BI DDA" TR.SFIGLRATI IN MADONYE.

treolarmente alle sicule; cosicehè essa, come certi granchi, certi nicchi e certi pesciolini, impressi nelle piccole sculture ellenistiche. suggella dorigine marina i tessuti che la recano. Ma non alimentata d'alenn significato simbolico, nasce e muore nellangusto campo tessile indicato come un qua. lunque effimero capriceio ornamentale.

Diversa sorte eblue invece il simbolo einese Luen che ci off re un evidente esempio, fra i tanti. dell'attivo polere trasmigratorio di certi segni ideografici, di regione in regione. Detto simbolo è di tre sfere fiam- meggianti poste sovra fiore di loto, ad indicare, questo. la terra e l’acqua, quelle, il cielo.

Tale appare primieramente in Oriente in nua seta cinese del secolo VII, nel Tesoro di Nara (fig. $3 A$ ); ritorna quindi nel suo ulteriore cammino verso Occidente nel drappo ond’è covertata la tomba di una moglie di Murad II (1451), in Brussa; e poi in altri velluti, tappeti e maioliche osmanici; ma ivi in forma di tre palle e di ondulazioni. in luogo dellacquatico loto. In Italia il simbolo rifiorisce in molteplici allegorie cosmiche, come ad esempio su di 

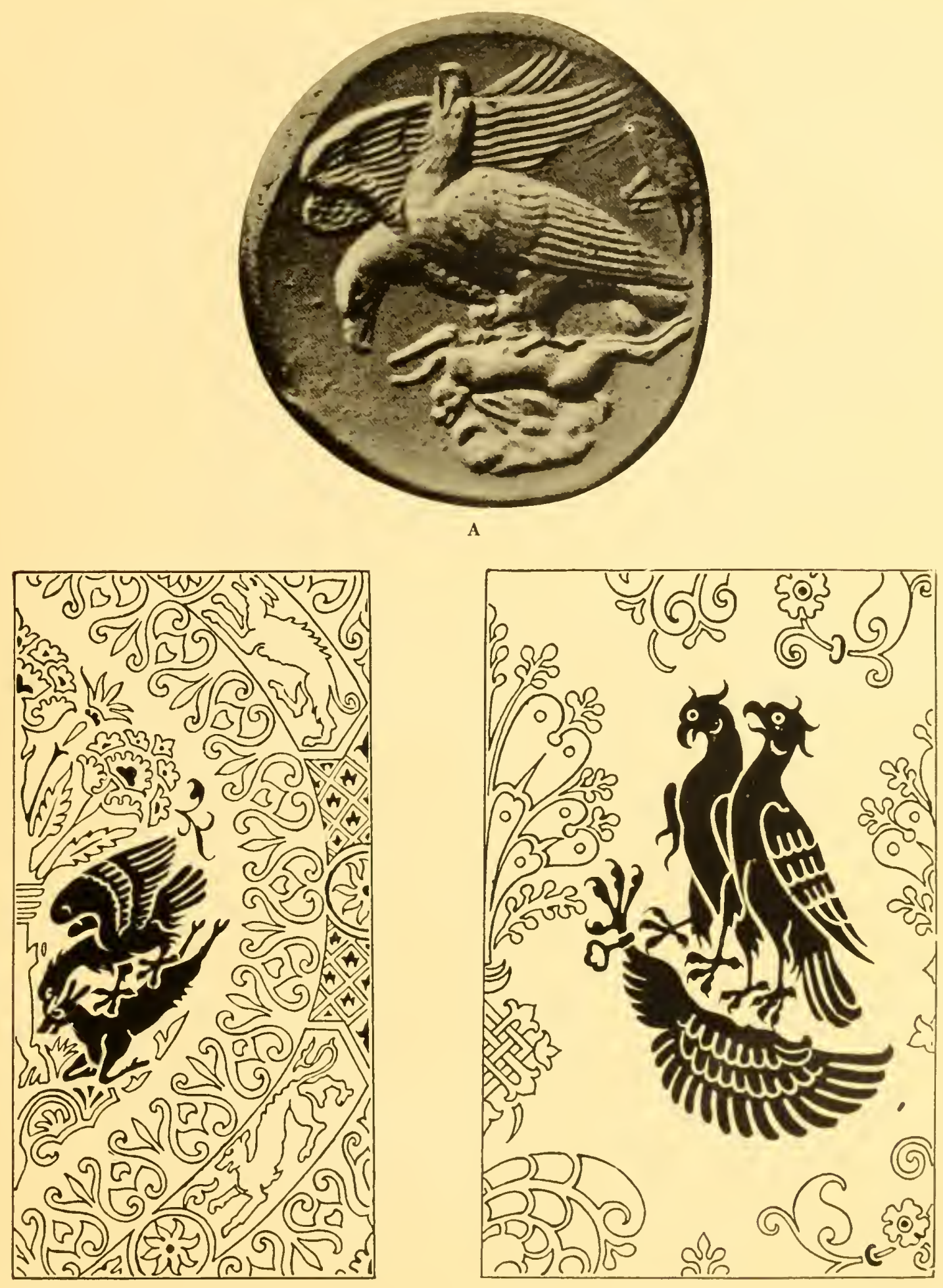

B

C 

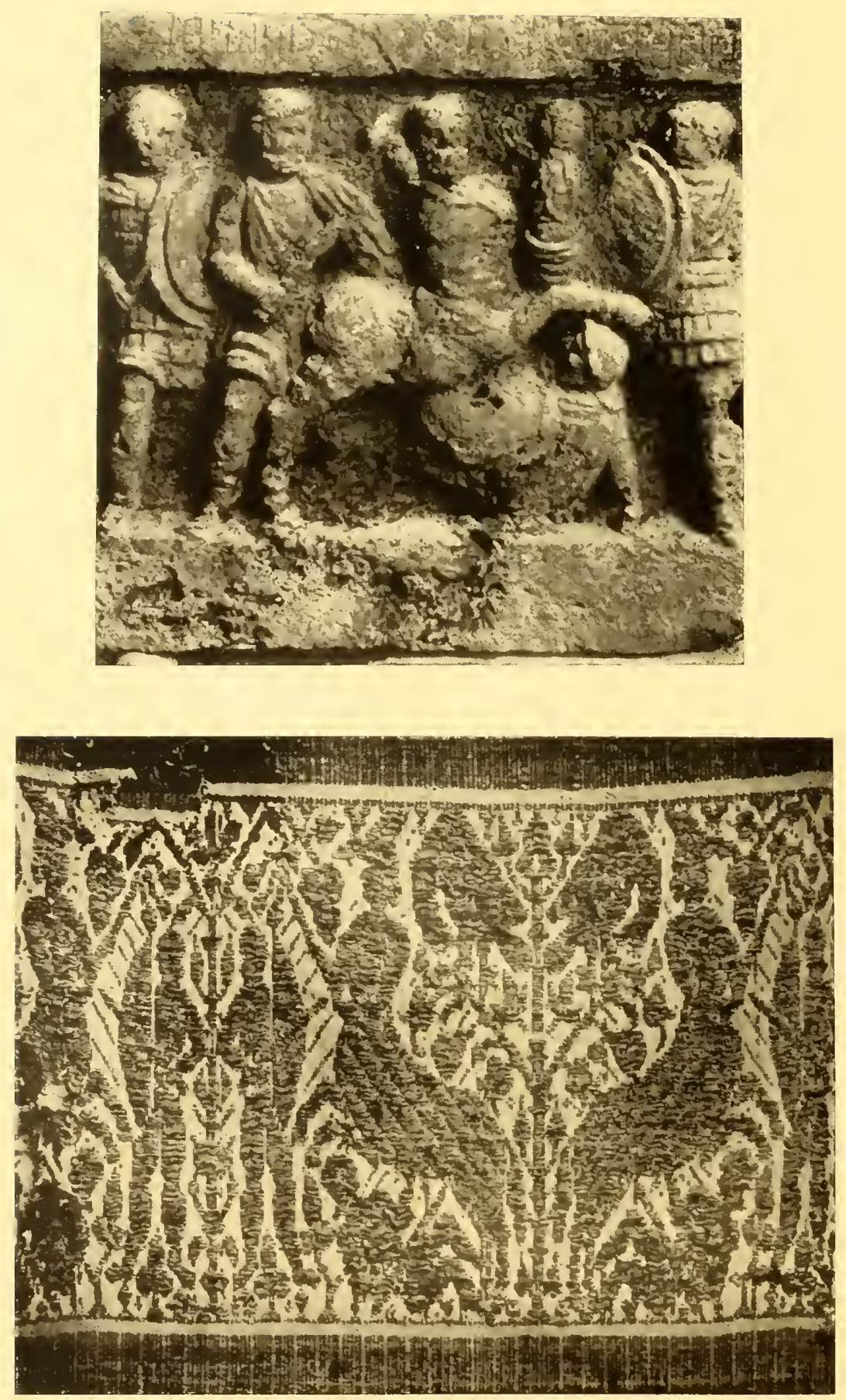

Fig. 6. - RAFFIGLRAZIONE SYOLTA DA UX'URNA ETRUSCA IN UNA TOVAGLiA PERUgiNa (dal W, Bombe). 


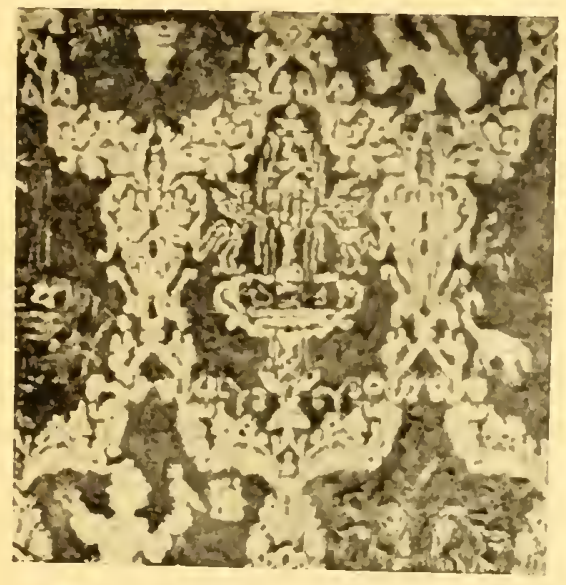

A

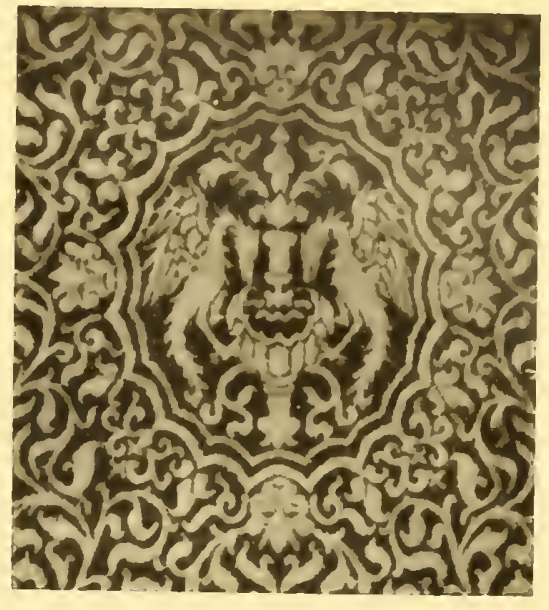

B

Fig. :- - "FONTANA DAMORE " IX TAPPETO DI PESCOCOSTANZO, CAYATA DA IX BROCCATO LLCCHESI.NO.

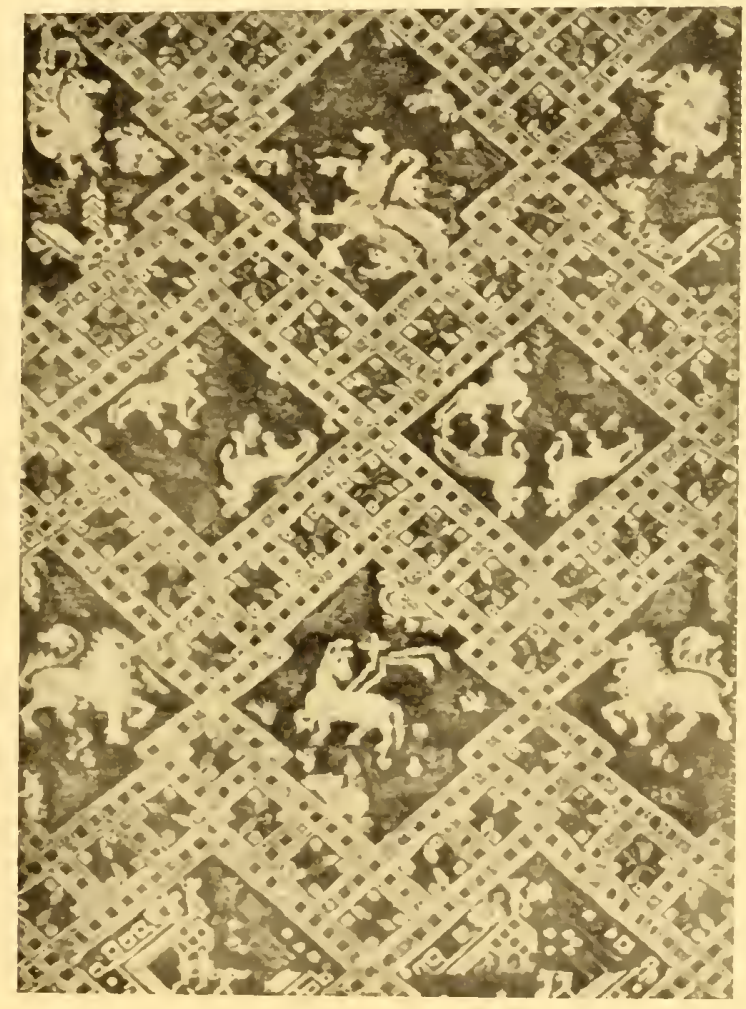

Fig. 8. - ELEMENTI PROMISCLI IN LX TAPPETO IBULZZESE 

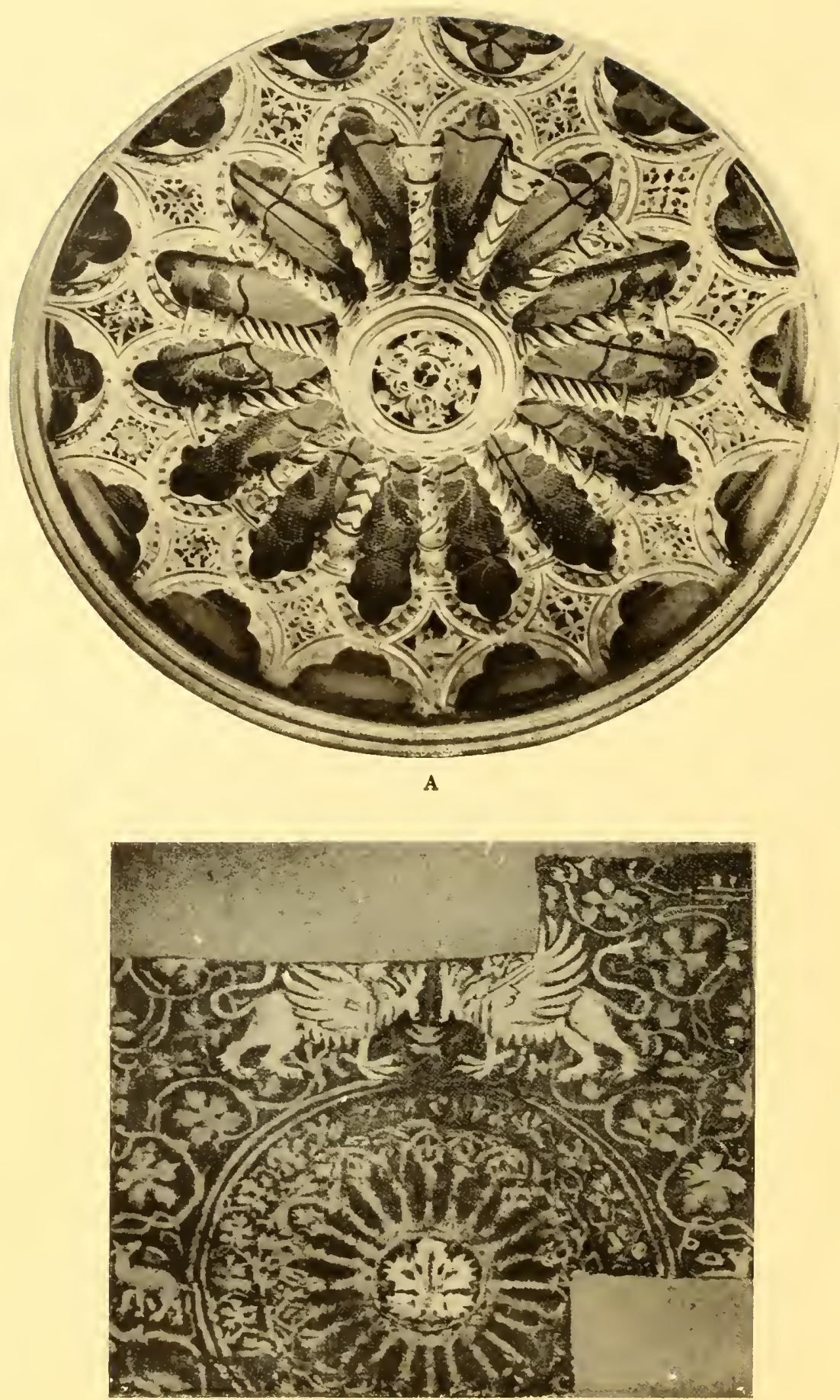

B

Fie, 9. - ROSONE ARCHITETTONICO TRADOTTO IN UN BBOCCATO TRECENTESCO. 

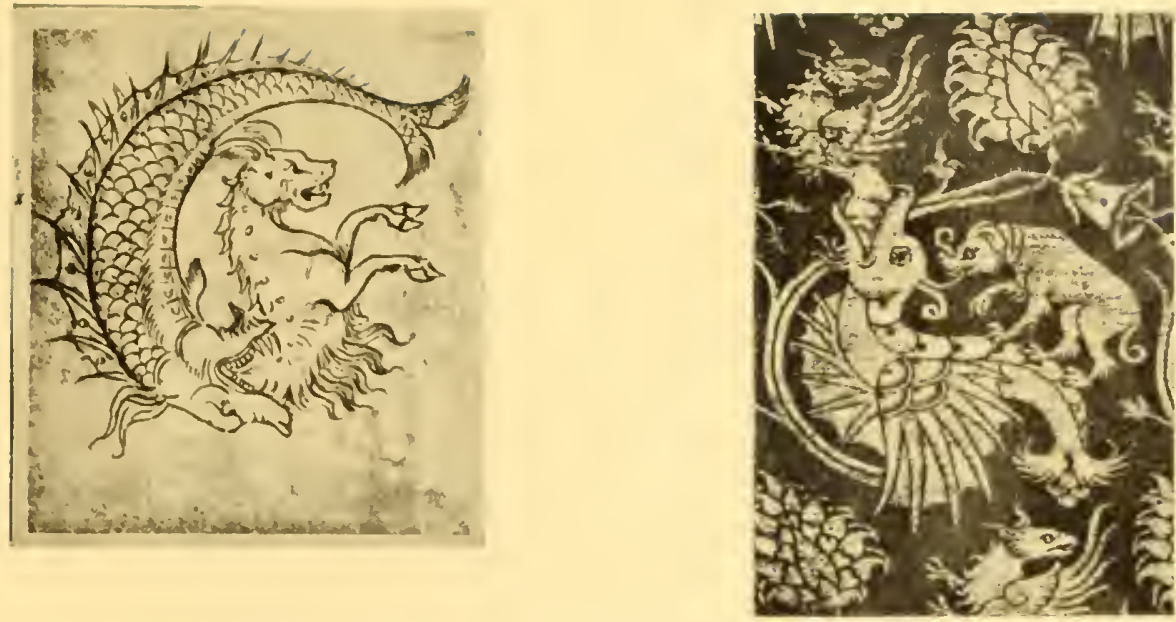

Fig. I0. DISEG

un broccato veneziano. recante dette sfere fra aquile raggianti verso leoni alheveranlisi (fig. $3 \mathrm{~B}$ ); poscia. corrotto. scompare da un ultimo drappo quattrocentesco. qual semplice motivo privo di contenuto (figura 3 C).

Forse per la stessa via. altrettanto tempo impiegarono le nuli stilizzate in nastri a raggiungere dalla Cina le anconette del Trecento nostro, che le accolsero nei loro cieli d'oro. E certi accenti stilistici d'elegantissima grazia nelle Madonne dello stesso tempo non consuonano forse lo stesso coi dipinti della dinastia Ming? Par rispondere all'ardita domanda il qui annesso hroccato tibetano raffigurante i Cento Budda, che vediamo poi trasfigurati in una teoria di Madonne, intessute al duodecimo secolo occidentale (fig. 4).

Ma a chi non volesse sapere d"influenze eteroclite (le quali non credo detraggano all'arte nostra neppur menomamente), riferisco in compenso due fonti indigene off erte dal sottosuolo archeologico. Eeco un deca- dracme di Agrigento con due atpuile che ghermiscono un eoniglio ( fig. 5 A): ed eeco qui in confronto due stoffe (fig. 5 B. C.). le quali famo pensare come la moneta stessa fosse per avventura capitata nelle mani di un mile tessiere che prese vaghezal abbellirue qualehe suo prodotto.

Piì evidente ancora i questaltro paral. lelo, riferito dal Walter Bombe (10). fra di un urna etrusea raffigurante la lotta dej Centanri contro i Lapidi. ed una tovaglia perugina dalla stessa seena (fig. 6).

Tanto meno raffinata is larte in genere, tanto piu trasparenti le fonti. Per esempio. il noto gruppo di tessuti rustici alıruzzesi. mostra evidente un intero repertorio di motivi spurii infiltrati lateralmente dallarte civica pin evoluta: cosi la fontuna d"amore zampillante su d"un tappeto di Pe'scocostanzo (fig. T A) echegrgia da un liroccato luechesino piì antiquo ( fig. $; B$ ). E come questa. le tante altre vellose stoffe del genere sono sorprese in flagrante plagio da alcuni migliori prodotti serici super- 

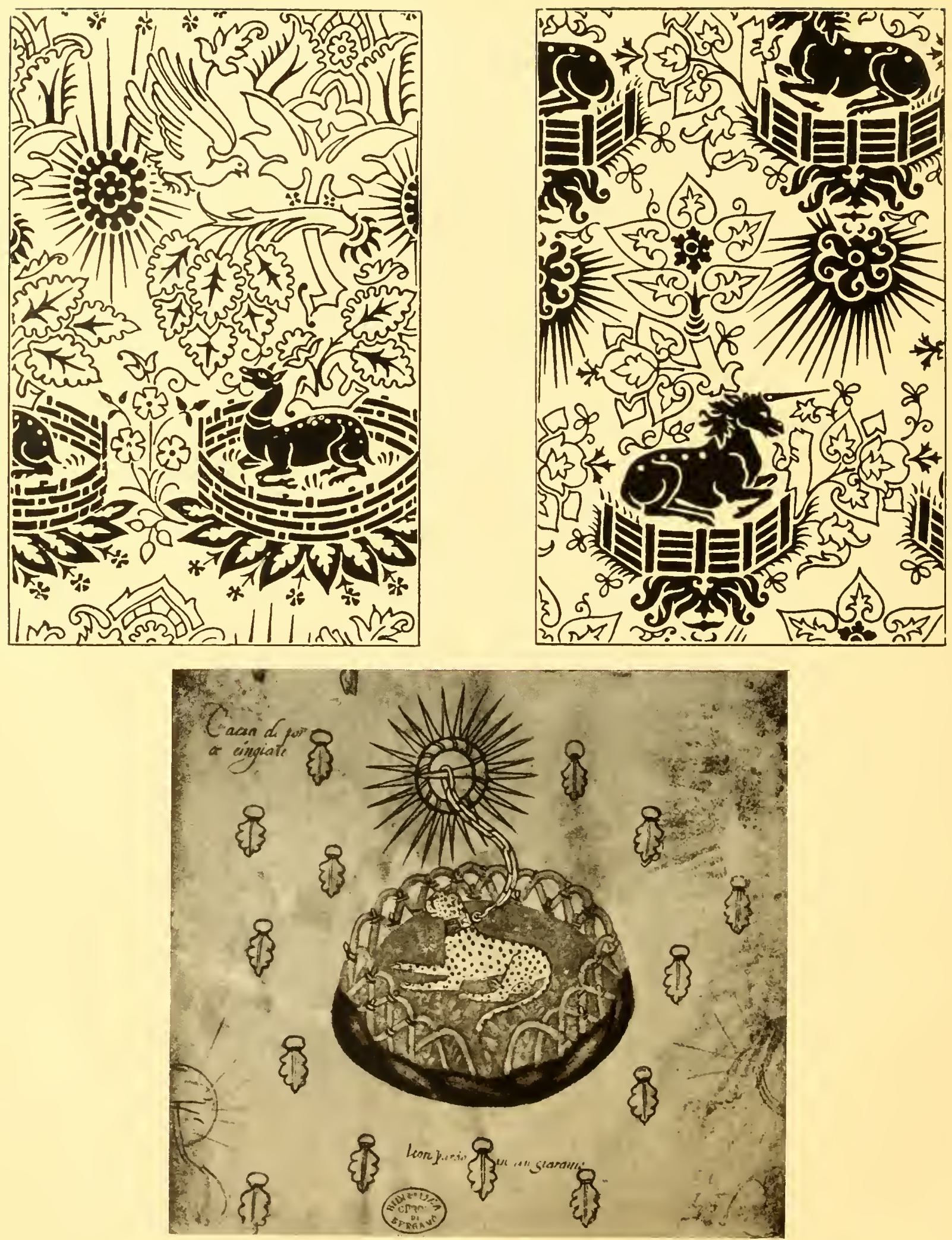

Fig. 11. - MOTIVI TESSILI E DISEgio ORIGINALE DI GIOVANNINo DE' GRASSI. 

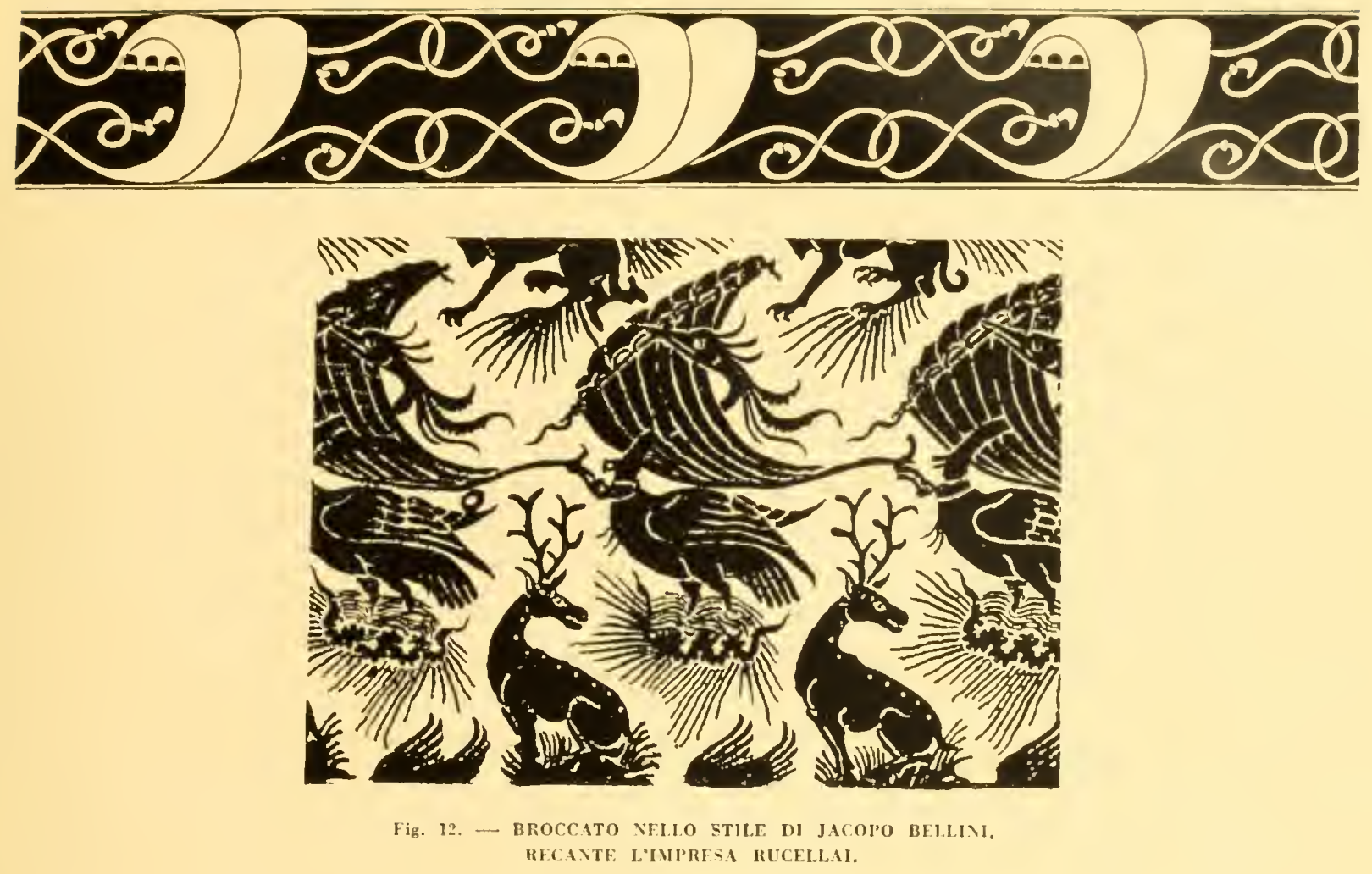

stiti: mentre altre ancora tradiscono anonime fonti interpolate ingemamente a forme arretrate. come nel tappeto abruzzese qui presso a fig. 8, cui ogni singolo motivo promiscuo potrebliesser rimosso o sostituito senza comprometterne l'incondito in. siene.

Al contrario. le vere opere d'arte, sian pure vasi, mobili o stoffe, hanno un loro proprio equilibrio, mantenuto da quel tanto quanto basta, e non tollerano nemmeno 111 soffio che ne alteri minimamente la compagine delicatissima.

A codeste siffatte fragili creature dei maggiori centri artistici torniamo adunque, dimostrando con l'esempio come in esse pure il plagio formale possa perdere il suo significato negativo.
L' architettonieo rosone gotico fig. $9 \mathrm{~A}$ tradotto da una facciata di rhiesa in 111 broceato del Trecento (fig. 9 B), vi appar in legittimo posseso, tanto armoniosa muita di disegno lo collega ai grifi a a viticei che lo circondano, quasi a farcene olliare lorigine estranea all'arte tessile.

Una tale virtuosa fusione stilistied di forme dissimili per destinazione, convien-i solo ad egregi artisti; dai quali. del resto. abhiamo altre e più sicure prove del lory amore per l'arte tessile. non solo conte materia pittoresca, sibbene cone campo di attività diretta.

Già altrove ei avemue di riconosece in tessuti la maniera. e talvolta la mano stessa, del Pollajnolo, del Ghirlandaio e di Pierin del Vaga; e qui looceasione di un disegno 


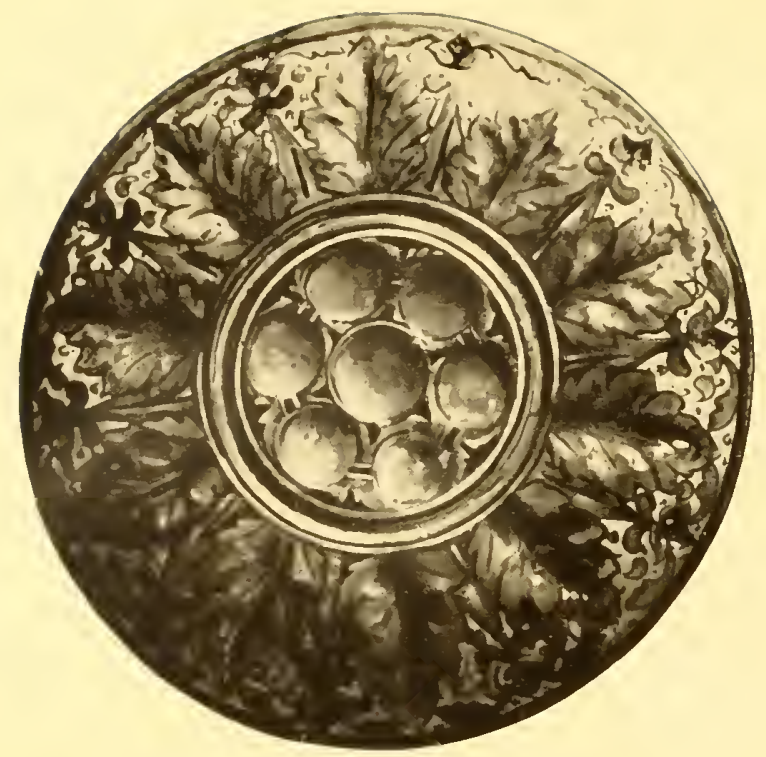

A

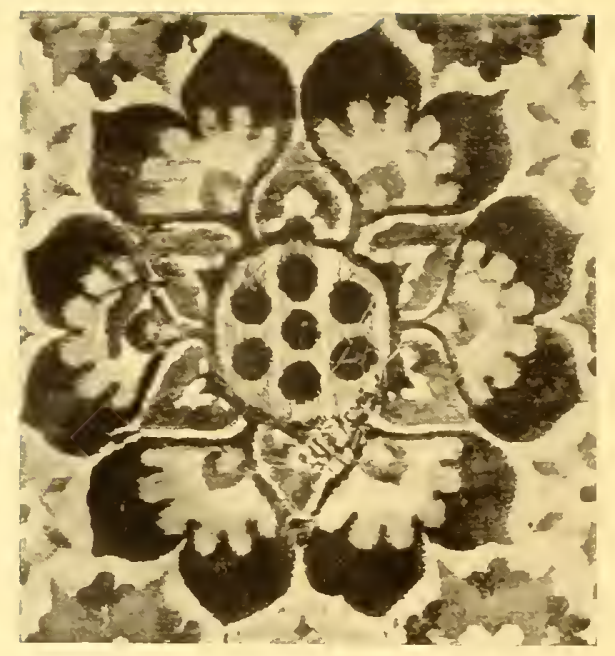

B

Fig. 13. - STEMMA MEDICEO IN UY DESCO E IN UY VELLLTO.

del Pisanello, messo dal Falke (11) a riscontro di un broccato che ne ripete il mo. tivo (fig. 10). cinduce, in linea comparativa, ad attribuire a Giovannino de" Grassi, in sulla scorta di un suo disegno nella Biblioteca di Bergamo, i due motivi tessili del leonpardo e dell'unicorno in campo palizzato a fig. 11. Cosi, se non il motivo, lo stile, ch ¿̀ come si dicesse piu, di due disegni di Jacopo Bellini, riferiti pure nel Falke (12). consuona col drappo fig. 12; il eni tema delle vele gonfie, passando ad altro, si riconosce in sulla facciata di Santa Maria Novella per limpresa Rucellai. svolta peró nel tessuto con cani, cervi e cigni, a significare le virtù che famo il mereante principe.

El ora siamo alle imprese. agli emblemi e agli stemmi. i quali furono preferiti in tutto il Quattrocento, e segnatamente caratterizzano d"araldico lo stile tessitorio; ma torua diffeile talvolta il discernerli nelle stoffe che li reca. tanto perfetta n'era la fusione nel contesto ornamentale, per non renderne l’iterazione. necessaria all’indefinito uso tessile. troppo ostentata.

Se linsegna medicea a sette palle non fosse altrove espressa nella precisa forma di elipeo posto al centro di un rosone. come in un desco engubino (fig. $13 \mathrm{~A}$ ). e in certe porte al Bargello, e nei pennacchi delle bifore del Palazzo Riccardi, stenteremmo a riconoscerla. cosi fiorita. in 11 magnifico velluto policromo che n’è tutto seminato senza voler troppo apparire ( fig. $13 \mathrm{~B}$ ).

Egualmente l’impresa dell’Arte del Cambio. chera di fiorini dioro in campo rosso. potreble passare inosservata quale m elementare motivo vestiario qualsiasi. insufficiente a determinare lepoea e il earattere emblematico del velluto ehe la reca ( fig. $14 \mathrm{~A}$ ). se essa non corrispondesse appunto. nella medesina continuità tessile. in sulla cornice del trittico degli Orcagna. negli Uffizii, raffigurante S. Matteo (figu- 


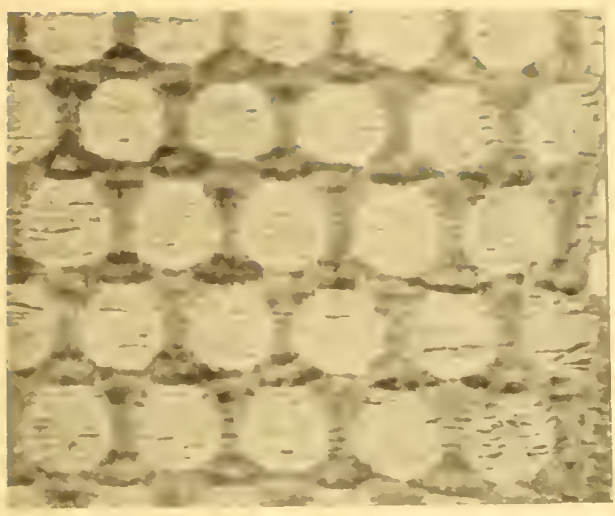

A

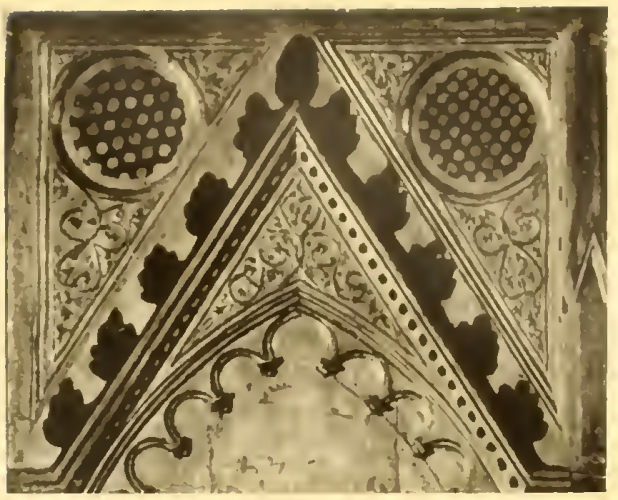

B

Fig. 11. - IMPRESA DHLLARTE DEL CAMBIO II LY VELLTO E MILLA CORYICE DI US TRITTICO TRECENTESCO.

ra $14 \mathrm{~B})$, postavi ad indicare la corporazione donatrice. Inoltre nel presente velluto conferma l’identificazione dell’impresa la specie membranacea delloro, sincrona al dipinto, e il preciso diametro dei dischetti aurei collimante coi forini mezzi ed interi.

All un'altra emblematica indagine ci avvia un caratteristico gruppo di fregi a geometrici intrecci. destinati in gran parte ad ormare piancte e mitrie (fig. 15). quale quella di Bonifacio VIII in Anagni e altri paramenti dello stesso tempo. i più. conservati in cattedrali franche e renane.

Di ornamentazioni geometriche ve ue hanno tre lipi etnici distinti: il celtico, forito di fantastiche mostruosità; l'arabico, matematico e coloristico; il vinciano, esteticamen. te ingegnoso. Ma ron nessuno dei tre riquadra il nostro per la tipiea irregolarita meandrica costantemente inscritta in formelle quadre, qual simbolo piì the non puro ormamento; e la sua presenza in indumenti di preciso uso liturgico condiea piuttosto una prohahile significazione sacra, avvalorata dalla coincidenza, in molte chiese dellalto medioevo. e massime in encelle delle regioni menzionate, del motivo labirintico, sia seolpito in lapidi, sia jutarsiato in pavinenti, secondo la sua tradizionale forma mitica, con però seguata al centro la perta di Gemsalemmese fore a significare la difficile meta celeste della vita, per la dubliosa via terrena. simile appunto alla favolosa dedalea.

Ma se il simbolo ha le sue originazioni logiche lontanissime. cosi pure il mero ornamento hat talvolta il suo etimo estetico piì o meno remoto.

Uno dei motivi piò frequenti uei drappi ispano-moreselhi verso la fine ded Duattro-

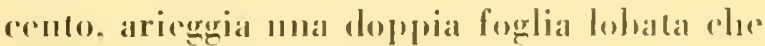
si direbhe. di leggeri. estemporanea creazione di un qualche fantasioso disegruatore (fig. 16 A). Orbene. is desso invere un serolare derivato attraverso la passiva riprodu. zione della coppia di pappagalli affrontati (fig. $16 \mathrm{~B}$ ). cari alle stoffe arabiche: la quale col tempo. avendo perduta la sua prese cisa forma ornitografica ne di ora la sola sensazione impressionistica.

Nella stessa guisa. inconsapevolnente, per 

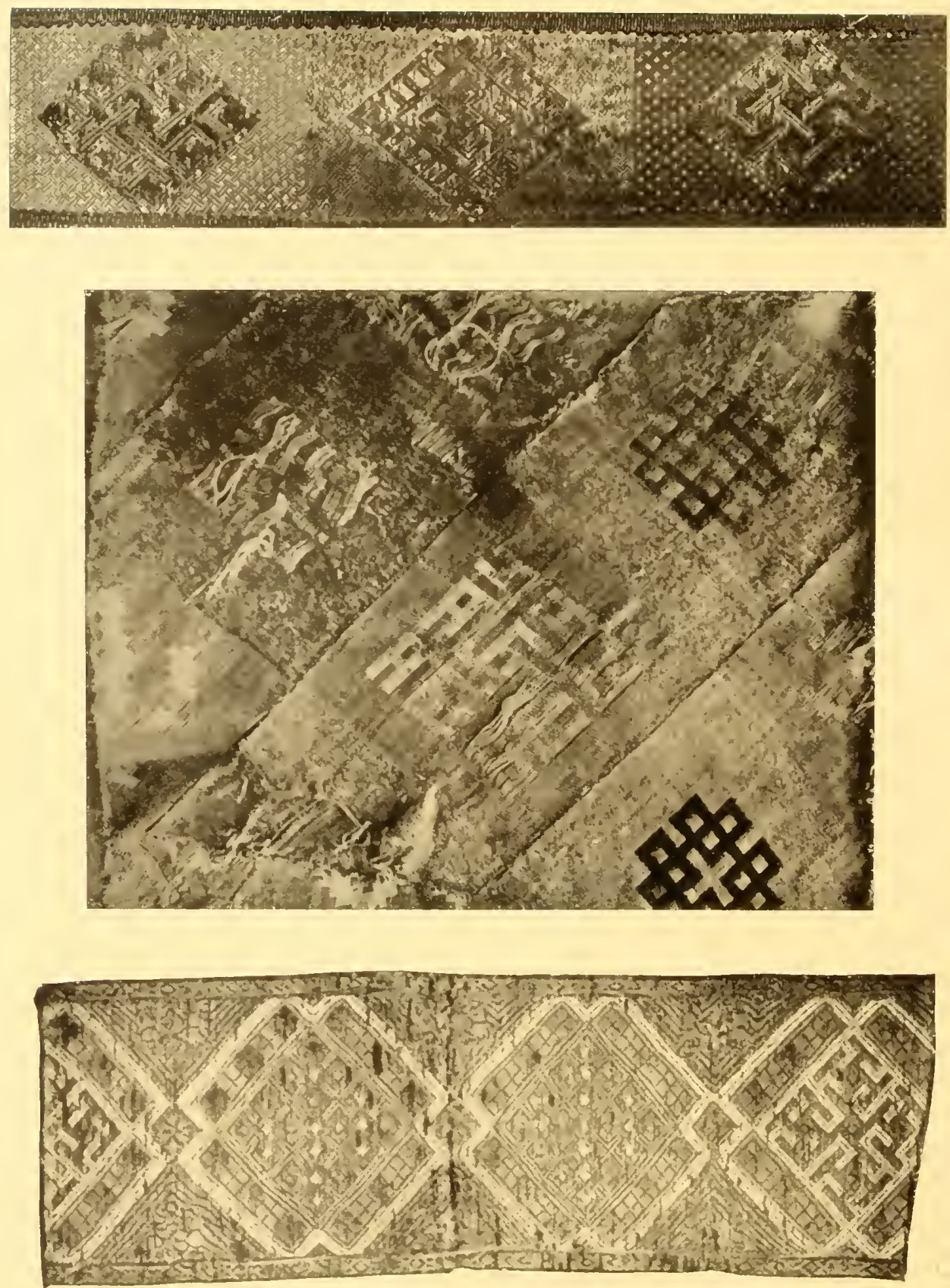

Fig. 15. - ORNATI LABIRITICI IN STOFFE LITL RGICHE

gradi impercettibili. attraverso la prevalente corrente coloristica medioevale, si sono formati i motivi amorfi da realistica origine: come con processo inverso la più mirahile Madonna di Raffaello si può rite. nere, seguendo il Darwin nell esempio, una felice risultanza di casuali incertezze pittoriche, dovute a remote generazioni di artisti, niun dei quali intese in principio neppur disegnare l"umana figura. 


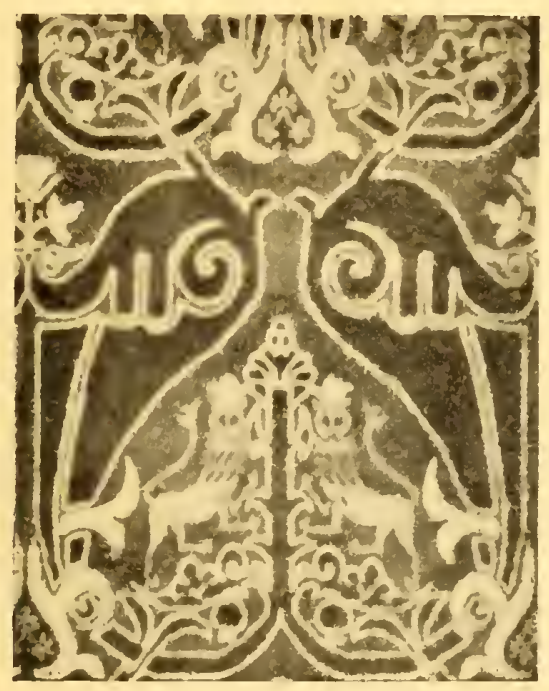

A

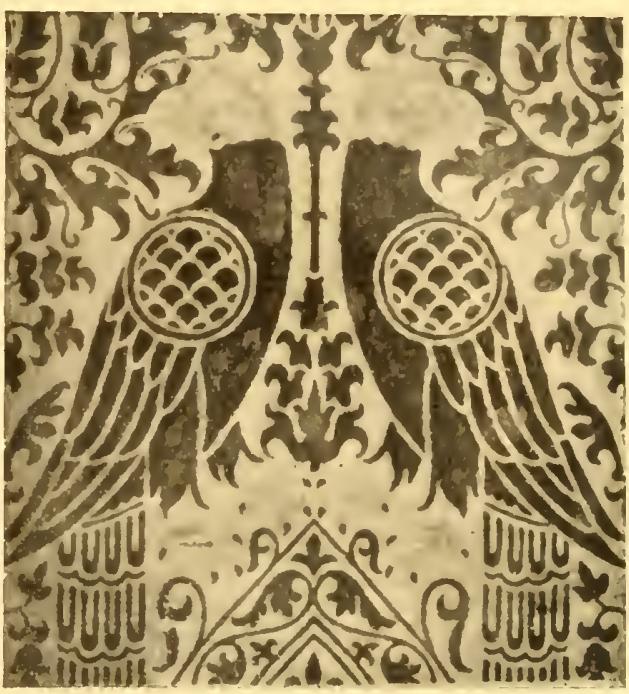

B

Fig. 16. - CORRL ZIOUF DI LN MOTIVO OIITOGRAFIIO

Con le discorse osservazioni non intendiamo aver passata in completa rassegna la ricchissina flora di simboli, emblemi e nolivi tessili. in gran parte commni ai vari generi artistici: bensi abbian solo voluto sof-

11) DALTON. Bysantine art and archeology, Oxford. 1911 , pag. 692.

(2) STRZYGOWSKI. in Jahrbuch der $K$. Preus. Kunsts., 1913, pag. 158.

(3) FALKE, Kunstgeschichte der Seidenueberei, Berlin, 1913, vol. I, fig. 69.

(4) GAYET, Exploration des nécropoles gréco-byzan. tines d'Antinoe, in Annales du Ulusée Guimet, 1. XXX, p. 37 . fermarci ad aleune -pecie piò caralteristich" e proprie allarte nostra. mirando identificarle secondo lo spirito più che non la propria forma estrinseca.

151 ERRERA. Collection dinciennes itoffes égyptien. nes, Bruxelles, 1916, n. 127 e 152.

(6) BOCK. Die Kleinodien.

(i) Melanges d'Archeologire. $185 \mathrm{I}$.

(8) DRECER, Europaische 11 eberei, wol. 1, 1:il. 33.

(9) Le Gallerie Nazionali ltaliane, vol. III.

(10) BOMBE, in Rassegna d"Arte, amo II. fa-c. V.

(11) FALKE. op. rit. vol. I1, fig. 112.

(12) FALKE. op. rit.. vol. II, fig. 484. 

ARAZZI CINESI 


\section{ARAZZI CINESI (*)}

Confesso di non avere una grande simpalia per laarte dellarazzo. quando. dopo il quattrocento. diventa pittorica. nè per quella musiva. quando, dopo l’epoca bisantina, subisce listessa sorte. Decadendo entrambi, III tecnicismo umiliante e meccanico piegasi in esse ad atrofizzare con punture d"ago, o a sezionare in tasselli, la vita che libera scorre nelle pitture di getto: e la virtuosa tortura non ripara il senso estetico offeso.

Nondimeno, codesti arazzi cinesi invitano e maggior indulgenza, anche se pittorici. e allammirazione, sempre che siano ornamentali: giacchè la loro tecuica è mezzo direlto d'espressione e pregio intrinseco alla hellezza stessa; mentre la loro lingua cromatica non potrehliessere espressa alirimenti. rivelando unantica nobiltà avezza ai tripudi delle tinte, ma parsimmiosa nell, spenderle; onde i rossi non mai vi offendono i verdi, i gialli non gli azzurri, i viola non $i$ ranciati...

Antica nohiltà e più remote origini elolve lindustria tessile cinese in genere; ma scarse ne sono le nolizie a noi; scarse le reliquie di certa datazione; lentissime le nutazioni estetiche; insensibile ai tempi la teenica; e però premature le classificazioni, incerte le attriluzioni.

Gli unici punti di riferimento cospicui (ma lontani fra loro tanto che dall'uno non si scerne l'altro) ci vengono offerti dalle recenti invenzioni tombali dello Stein nel Turckestan; dal tesoro tessile di Nara in

(*) Dalla Russegna d'Arte.
Giappone: da poche sloffe mperstiti fra le mole importate in Oceidente durante la dominazione mongoliea. alcune drlle quali di cerla data per destinazione storica provenienza: poi da nua grande quantita di tessuti flutuanti nel mare magnum delle antichita furate e slandate dai repressori dei Boxer. i fuali tessuti risalgono in mas. sima parte al più recente periodo $K^{n}$ ien Lung.

In codesto periodo, per la loro varia copia. richiamano l'attenzione. distingnendosi nella tecuica dai broceati e dai velluti, appunto gli arazzi, detti in einese lio ssen.

Svolti in seriche tinte piane su temi fantastici, pieni di riposti significati, ispirano un senso religioso e strano; e la loro spontanea finezza invita a studiarli partitamente da vieino. per scoprirme $\mathrm{i}$ segreti delloperil tessitoria, per osservarme le particole dove il pesmello acuto ainta e contimna il magistero dellagon. e per trovarvi intessute con looro di varia intonazione (or freddo, or caldo e or quasi molile in 111 senso o in um altro. secondo il ginoeo delloordito). perfino cangianti peme di pavone.

Ci si accorge cosi di tante miunzie utili a serverare le diverse tecniche. spesso confuse. o quasi sempre spieqate con inverosimili procedimenti. Si i detlo. per esempio, e si ripete ancora. dhe codesti arazzi. sono composti di tanti piceoli tasselli néssili. tessuti uno ad uno. e rimbiti insieme a mo della veste d"Arlecehino: mentre invece, pereorrendo con ocelio altento la trama, non vi si notann intermzioni di contimuità, 


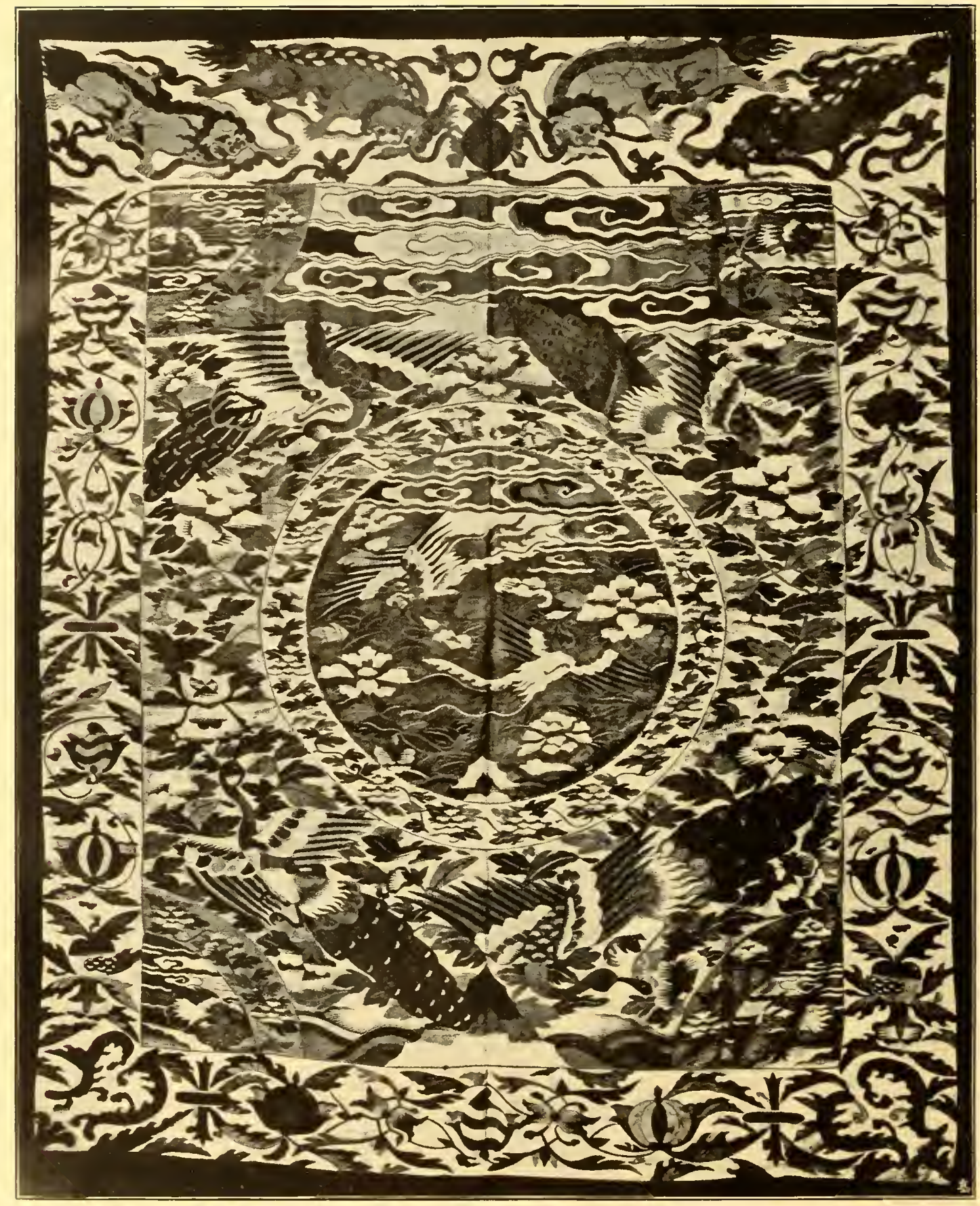

Fig. I. ARAZZO (INESE DEL PERIODO K"IEY LLYG.

bensi su d’essa si può segnire la gugliata che va e torna serpeggiando a colmare i campi d'ogni colore singolo.

Or del loro ufficio pratico. I più piccoli, quasi arazzi in miniatura. simili a kakemono lunghi e stretti. ornavano i tempii d'imagini e scene hnddiche; e i maggiori, come quei famosi nel Museo Britannico. le

124 


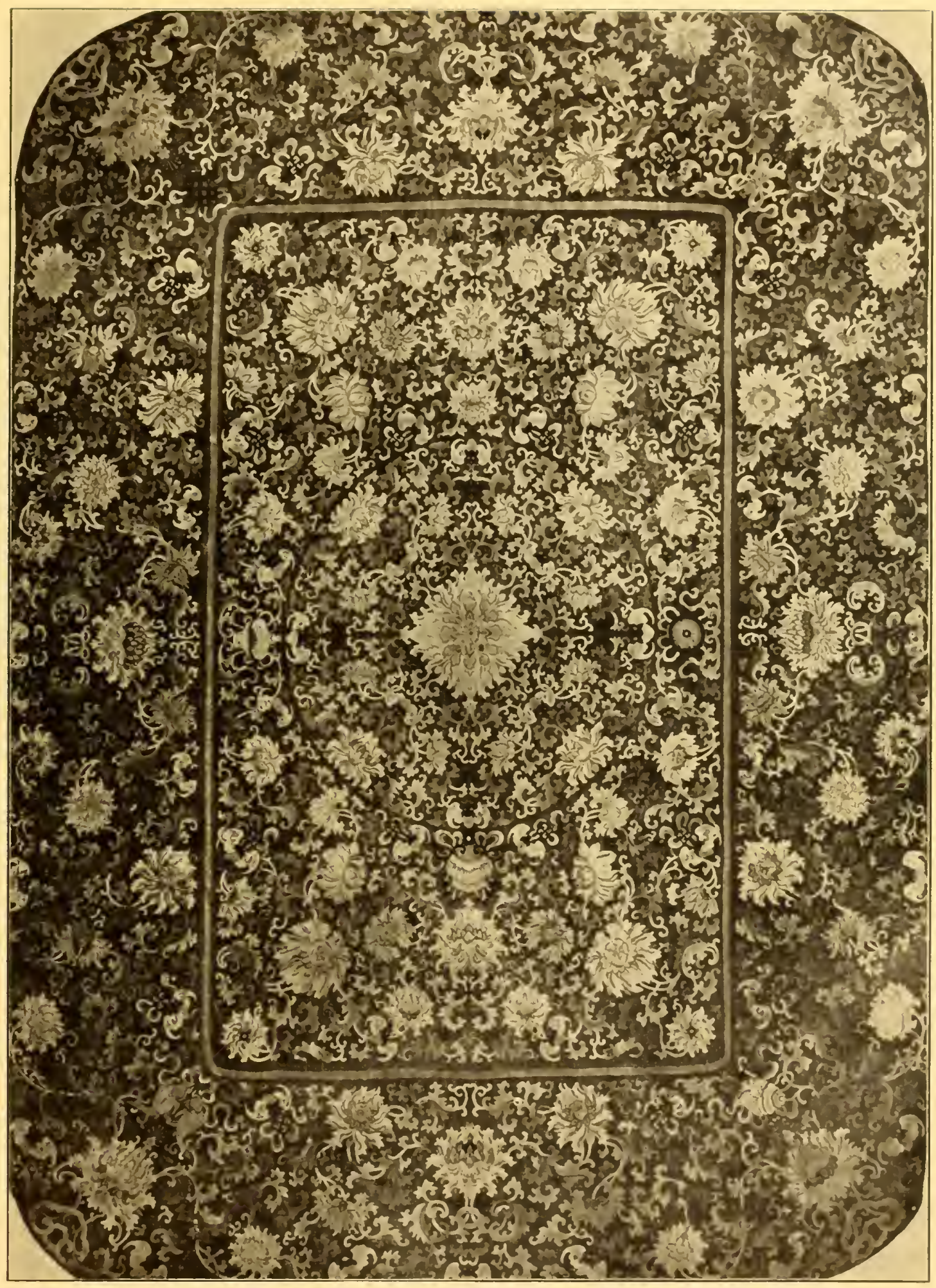


sale imperiali, commemorando feste solenni, o anticipando con realismo terreno le delizie del paradiso taoista.

Altrimenti, per la loro propria lasca te. stura, obbediente alle pieghe flessuose del gesto. se ne facevan vesti sacerdotali e civili; spesso, le une. con gli otto noti simboli di buon augurio e con rattoppi simulanti la miseria predicata da Budda; e talvolta, le altre. con l'allegoria cosmica dei quattro elementi o con la sigla della longevità.

A codesto rifiorire dell"industria arazziera asiatica non sembra a caso corrispondere in Occidente, nello stesso meridiano di tempo, quella dei gobelins: industrie entrambi cortigiane negli intenti e mn po' servili nei propri mezzi tecnici.

Infatti se agli arazzi copti, capostipiti del ramo industriale occidentale, si può attribuire origine asiatica (e perchè no, se questi anteriori ai tessuti che ne rappresentano già un meccanico perfezionamento, accertato di discendenza cinese?), riesce facile riallaceiare le fila delle due tradizioni, appunto dall'Egitto greco-romano in su, per il tramite arahico, per il medioevo bizantino e romanieo, e via via dal rinascimento nostro al settecento francese.

In tal guisa, rotti i ponti nel procedere, le due industrie sorelle trovaronsi isolate nello spazio a un medesimo grado di sviluppo pieno, riflettente ognuna lo spirito delle civiltà diverse in cui si maturano, immemori della comune origine; ma prima ch'esse volgessero in decadimento, per poi estinguersi, interviene un fatto storico, che sebben contingente. pare avvicinarle quasi in allegorico comiato.

La reale manifattura di Beaurais soleva ogni anno offrire in omaggio alle corti stra- niere una serie di arazzi; e nel 1763 , da parte di Luigi $X V$. sei. su cartoni di Boucher, ne toccarono a $\mathrm{K}$ ien Lung. Il quale li ricambiò con altri di propria fabbricazione. di cui uno superstite nella raccolta Larkin di Londra, rappresentante una festa dinastica alla corte del menzionato imperatore ( fig. 3). Egli vi appare seduto al desco con la consorte ed i figliuoli. mentre uno di loro gli offre uno scettro, ed altri fan concerto. Una cornice di puro stile francese, copiata agli stessi arazzi donatigli. riquadra la scena.

Del resto. codesto esotismo è un fatto comune all'arte tessile antica: la quale fu sempre tramite diretto dinfluenze remoie, recando lungi, quali germi contagiosi, segni di novelle corruzioni estetiche, per le facili vie del commercio marittimo. Ma se il nuovo spesso isterilisce in terreno popolare, incubato nelle corti, indi si propaga più facilmente.

Il gusto per l'egizio presto si diffuse in Roma da quella di Adriano Imperatore, e appunto, meglio che non gli obelischi, furono i vela alexandrina a promuoverlo: prima le vesti seriche ai principi, poi, ai volghi: bilingue l'alba dell"incoronazione di Guglielmo II di Sicilia: con draghi cinesi e dedieatoria araba. una delle settecento pezze offerte dal Gran Khan mongolo al Califfo Muhammed Nasir: con scene cristiane fra ornati persiani. i tappeti dello Shià Abbas alla Repubblica Veneta: e con cornici francesche, finalmente. gli arazzi dı Kien Lung a Luigi XV.

Lasciando le corti mezzane all'arte, sof. fermiamoci ad ammirare quest'altro tipico arazzo cinese. il cui disegno, come sommerso in onde di colore vibranti da pavoni, fe- 


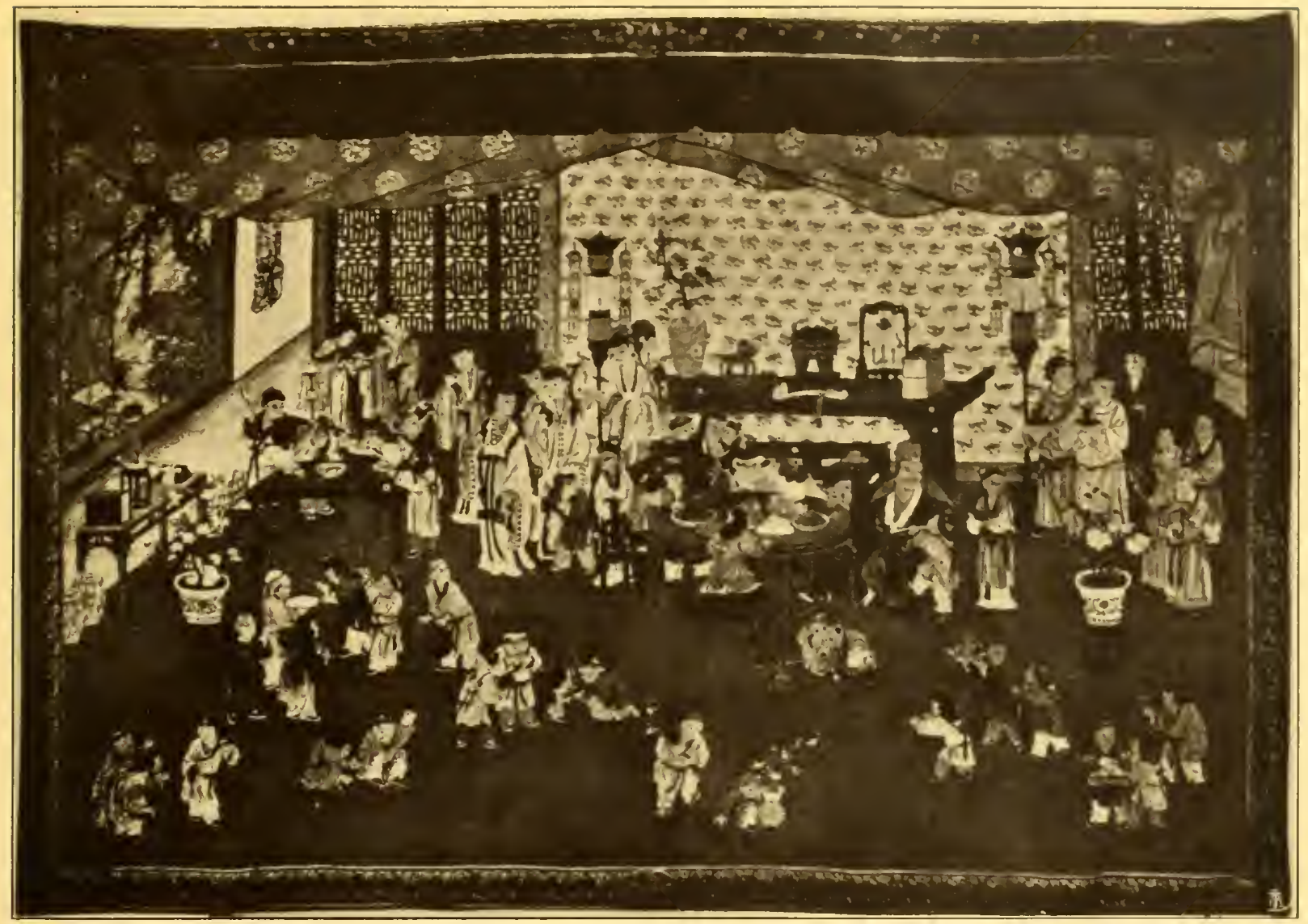

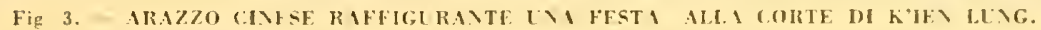

nici. pronie e muhi, confondesi in eguale ritmo (fig. 1).

Quanta poca importanza abbia ivi il tema in rapporto alle tinte. lo dice la spera al mezzo, che riflette il più ampio motivo del fondo: e lo ripete. nella stessa guisa il seguente tappetino tessuto a coprire una mensal sacra a Bulda (fig. 2).

E desso tutto un fior di loto pallido in campo azzurro fra simboli augurali. Il folto fogliame vi ì ombreggiato con tre grali di versle. il chiaro. lo seuro e il lor tono me. dio: e questa riduzione cromalion. normale nei periodi coloristici. anche fuori Cina. rende magrior rilicvo che non $\mathrm{i}$ lecehetti dello sfumino accademico.
Pur notevole nellinteson drappo. un particolare comune allarte cinese tutta. cioè gli angoli smussati. Smussati sono gli angoli alle scatole di lacca. ai mobili e ad ogni oggetto diso consueto. per renderli dolci al tatto quanto $\mathrm{i}$ velluti. maneggiandoli: e in senso traslato delle cose tangibili. gli smusoi passarono a quelli della vista; la quale pur vuol rssere accarezata. non amando le punte.

Sono sottigliezze. lo so. ma che nel loro complesso adombrano l"ineffabile essenza dell'arte orientale, congegnata appunto di sottigliezze sottilissine: non mai però inutilnente pazienti quanto in rerti odicrni ri(anti monastici. 
I N D I C E

Del rolore e dello stile nelle stoff's anticbe... . . par. II

Oro testile e sagrio trenico-analition . . . . . . . . . . . . . . 23

Le stolle e le vesti tombali di Cangrande fo della scala . . . . . 35

Velluti aulici. . . . . . . . . . . . . . . . . . . . 61

Reliquie tessili rinvenute nella tomba di sigismondo l'andolfo

Malatesta in Rimini. . . . . . . . . . . . . . 75

Tessuti figurati forentini . . . . . . . . . . . . . " " 8 -

Considerazioni sopra alcuni motivi e simboli tessili . . . . . . . 10.

Arazzi rinesi . . . . . . . . . . . . . . . . . . . . . 123 




